\title{
TRANSMISSION AND DETECTION TECHNIQUES FOR INTERNET OF BIO-NANO THINGS APPLICATIONS WITH STATIC AND MOBILE MOLECULAR COMMUNICATION: A SURVEY
}

\author{
Amit K. Shrivastava ${ }^{1}$, Debanjan Das ${ }^{1}$, Neeraj Varshney ${ }^{2}$, Rajarshi Mahapatra ${ }^{1}$ \\ ${ }^{1}$ Department of Electronics \& Communication Engineering, International Institute of Information Technology Naya \\ Raipur, ${ }^{2}$ Wireless Networks Division, National Institute of Standards and Technology, Gaithersburg, MD 20899 USA
}

NOTE: Corresponding author: Neeraj Varshney, neeraj.varshney@nist.gov

\begin{abstract}
Recent studies have shown that designing communication systems at nanoscale and microscale for the Internet of Bio-Nano Things (IoBNT) applications is possible using Molecular Communication (MC), where two or multiple nodes communicate with each other by transmitting chemical molecules. The basic steps involved in MC are the transmission of molecules, propagation of molecules in the medium, and reception of the molecules at the receiver. Various transmission schemes, channel models, and detection techniques have been proposed for MC in recent years. This paper, therefore, presents an exhaustive review of the existing literature on detection techniques along with their transmission schemes under various $M C$ setups. More specifically, for each setup, this survey includes the transmission and detection techniques under four different environments to support various IoBNT applications: (i) static transmitter and receiver in a pure-diffusive channel, (ii) static transmitter and receiver in a flow-induced diffusive channel, (iii) mobile transmitter and receiver in a pure-diffusive channel, (iv) mobile transmitter and receiver in a flow-induced diffusive channel. Also, performances and complexities of various detection schemes have been compared. Further, several challenges in detection and their possible solutions have been discussed under both static and mobile scenarios. Furthermore, some experimental works in MC are presented to show realistic transmission and detection procedures available in practice. Finally, future research directions and challenges in the practical design of the transmitter and receiver are described to realize MC for IoBNT health applications.
\end{abstract}

Keywords - Detector design, Internet of Bio-Nano Things, modulation schemes, molecular communication, micro-scale and nanoscale communication

\section{INTRODUCTION}

Molecular Communication (MC) is one of the fastest emerging research fields in the recent times where chemical signals are used to communicate between the transmitter and the receiver. It is worth noting that $\mathrm{MC}$ is a natural phenomenon and has been used by micro-organisms such as bacteria to communicate among themselves [1]. However, recent advancements in the field of nanotechnology enable the development of nano-scale devices that can also utilize MC to support several potential applications where conventional wireless communication using Electromagnetic (EM) waves is not feasible. Apart from this, biological cells can also be synthetically modified or generated to develop bio-nanomachines to support various applications within Internet-of-Bio-Nano Things (IoBNT) where the information can be easily encoded using the concentration, release time, and type of molecules.

It is important to note that since the capabilities of individual bio-nanomachines may be limited to simple sensing and actuation, the IoBNT [2] is envisioned to enable the interconnection of several bio-nanomachines to perform complex tasks. Applications of IoBNT include intrabody sensing and actuation, gene therapy, intra-body connectivity control, artificial blood cell production, and human body monitoring by an external healthcare provider
[2]. This paradigm also poses several research challenges in terms of communication and networking using biochemical infrastructure while enabling an interface to the Internet. Development of efficient and safe techniques for information exchange, interaction, and networking between the biological nano-machines within the IoBNT, is one of the major research challenges. In this context, MC has attracted significant research attention to support several health applications. Some of the other important applications of MC i.e., Lab-on-a-Chip (LOC) devices [3], [4], Targeted Drug Delivery (TDD) [5], [6], and the diagnosis and mitigation of infectious diseases at the cellular level [7], [8], [9] are described below.

The detection of biomolecules for LOC has been proposed in [3] using the Radio Frequency inductance capacitance (RF LC) resonator. The principle behind the biomolecule detection was detecting the changes in the RF signal due to the permeability and the resistance of the biomolecule. For LOC application, a relay-assisted MC based on dielectrophoresis was proposed in [4] where the molecules propagate from the transmitter to the receiver under the influence of a periodic electric field generated inside the relay. In this work, the inter-relay segment is modeled using the transmission line technique in which the relay provides drift to the molecules in the direction of the intended receiver. In [10], a wearable susceptometer (on finger) for detecting magnetic nanoparticles has been 
proposed. In this work, a nano-machine beneath the finger (inside body) could sense one type of molecule and release another type of molecule (i.e., magnetic nanoparticles), which can be detected by the wearable susceptometer. The principle behind this system is that the current through the coil changes if the magnetic nanoparticles pass through it. The equivalent circuit for this detector using the induction and the operational amplifier has also been presented therein.

A novel method was proposed in [11] to transmit the digitally encoded Deoxyribonucleic Acid (DNA) using the bacteria-based nano-networks. In this work, motile bacteria moved towards non-motile bacteria that stored the digital information using plasmid (a DNA molecule). The motile bacteria then conjugates with the non-motile bacteria to pick up the stored information. Finally, the motile bacteria deliver the information to another location. The movement of the motile bacteria was governed through a molecular positioning system containing trilateral beacons, emitting chemo-attractants.

For TDD applications, the authors in [5] proposed a method for target delivery of nano-sensors by using the bacteria-based coordination. Their proposed method utilized multi-hop communication along with two different chemicals for guiding the nano-sensors in a particular direction. This setup can be useful in TDD applications where drug delivery inside the cancerous tumor is required. Further, for TDD and monitoring the drug concentration, an implantable drug delivery system was proposed in [6] where the anti-cancer drug molecules were released by the transmitter towards the cancer cells that may form a lump or tumor. The drug molecules are assumed to propagate inside the Extracellular Space (ECS). For analysis purposes, spherical transmitter and receiver models are considered and the Channel Impulse Response (CIR) is derived and verified through particle-based simulation. In this work, the drug concentration profile at any point inside the tumor is also obtained by solving the convolution of the release rate and the derived CIR.

Furthermore, a scheme for reducing the effect of propagation delay in a simultaneous drug delivery system was proposed in [12], where a controller nano-machine sent signaling molecules to the drug-carrying nano-machines which could be at unequal distances from the controller nano-machine. Upon receiving the signaling molecules, the drug-carrying nano-machines were expected to release the drug molecules simultaneously. However, due to unequal distances from the controller nano-machine, the drug-carrying nano-machines could not release the drugs simultaneously. Apart from the TDD applications, various MC systems for health monitoring in IoBNT are also present in the literature. For example, the work in [8] proposed an MC-based health monitoring system which detects the biomarkers of heartattacks through the implanted nano-sensors. The nano-sensors were designed to sense the endothelial cells circulating in the body.
Also, the early detection of neurogenerative diseases such as Alzheimer's was presented in [13] considering the MC. This work considered an intercellular communication system where the calcium molecules were used as signaling molecules and their oscillation patterns were different in the astrocyte (biological tissue) of a person suffering from dementia in comparison to a healthy person. The difference between the other metrics such as the molecular delay, the channel gain as a function of distance (i.e., the number of cells between the transmitter and receiver), and the calcium oscillations were also presented. Further, a Microbiome-Gut-Brain-Axis IoBNT communication network was studied in [14]. Note that the MGBA refers to the bidirectional communication network between the brain and the gastrointestinal tract, which in general includes central, autonomic, and enteric nervous systems of the body, the gastrointestinal tract, and its microbiome (human microbiomes are responsible for launching the immune system, affecting inflammatory homeostasis and immune regulation). Also, electrical and molecular infrastructure to realize the communication through MGBA has been discussed in [14].

For monitoring the viscosity of blood, a wearable smart device was presented in [9] where a transmitter released molecules (in particular aptamers) inside the blood vessel through a needle and these molecules were received by the sensors. These sensors then sense the level of absorption and differentiate between the levels of absorption for high and normal viscosity scenarios. Further, a method for detecting cancer using mobile nano-sensors in the blood vessels was proposed in [15]. In this work, mobile nano-sensors are used to detect the presence of cancer biomarkers in the blood vessels. Finally, these mobile nano-sensors reach a fusion center (FC), which decides the presence or absence of an anomaly after detecting the concentration of biomarkers and comparing against the threshold using the Log-Likelihood Ratio (LLR) test. In all the above studies, the transmitter and receiver were either static or mobile under pure diffusive or flow-induced diffusive channels. Also, the scenarios and applications of MC systems differ in each of the works. Hence, a structured survey of transmission and detection under different scenarios and applications is required.

\subsection{Related works}

A survey on recent advancements in MC was presented in [16] in which microscale and macroscale communication along with modulation techniques, channel modeling, error correction codes, and simulation tools have been discussed. In [17], an MC-based nano-network has been studied for TDD applications. Particularly, the communication between different entities in a simple nanonetwork based TDD has been highlighted. Apart from this, several challenges in modeling the cardiovascular system, extracellular space, and cellular surface as a communication channel were presented. Apart from this, an 
overview of transmitter and receiver nano-systems has been provided in [17]. A survey related to transmitter and receiver architectures has been published in [18], where some of the modulation, coding, and detection techniques have been discussed. In addition to these techniques, a Graphene-based transmitter and bio-Field Effect Transistor (bio-FET)-based receiver have been described to realize the MC based systems.

A cooperative drug delivery system was discussed in [19] where multiple mobile nano-machines communicate and deliver drugs in cooperative manner. More specifically, the leader nano-machines sense the presence of a target and release the attractant molecules in the environment. The follower nano-machines subsequently sense the attractant molecules and move towards the target for releasing drugs. Channel modeling for an MC-based system considering different types of transmitters (e.g., point, volume and ion channel based), and receivers (e.g., passive, fully absorbing and reactive) has been studied in [20]. A survey on biological building blocks for MC has been presented in [21] where the transmitter and receiver for different types of signaling particles (i.e., cations, neurotransmitters, and phosphopeptides) have been described. This work also discussed the biological approach for Inter-Symbol Interference (ISI) mitigation. Further, a survey on modulation techniques for molecular communication has been presented in [22]. In [22], the modulation techniques have been classified as concentration-based, type-based, release-time-based, spatial techniques and higher order modulations. Moreover, the metrics used for evaluating the performance of modulation schemes are also presented therein.

In contrast to these existing survey papers, this survey paper comprehensively focuses only on the transmission and detection techniques present in the existing literature under different channel conditions. It is worth noting that the modeling of the channel is different for different applications. Therefore, this paper also classifies the transmission and detection techniques based on the applications. Broadly we discuss four different scenarios as shown in Fig. 1: (i) static nano-machines communicating in pure-diffusive channel without drift or flow, (ii) static nano-machines under a flow-induced diffusive channel which experiences diffusion as well as drift, (iii) mobile nano-machines under a pure-diffusive channel, and (iv) mobile nano-machines under the flow-induced diffusive channel. Further, we discuss the transmission and detection for relay-assisted-based MC systems, cooperative or distributed-detection-based MC systems, MIMO-based MC systems, and machine-learning-based MC systems under all four possible scenarios described above.

The first static transmitter and receiver scenario is shown in Fig. 2, where nano-machines reach the cell surface receptor and can communicate over the cell surface. Such a configuration of nano-machines is present in the application of TDD over a cell surface [23], [24]. Here the drugs can be delivered to the malignant cell while minimizing the drug delivery to healthy cells. Fig. 3 shows the static nano-machines bound to the endothelial cells. However, the signaling molecules reach the receiver via drift and diffusion inside the blood vessel. This case can be found in the application of blood viscosity monitoring [9] and other health monitoring applications. In Fig. 4 the nano-machines and the signaling molecules are mobile and follow Brownian motion (free diffusion). This scenario can arise in the TDD application within the Extracellular Fluid (ECF) [25] as the movement of transmitter/receiver within ECF is governed by free diffusion. Fig. 5 shows that the nano-machines and signaling molecules are mobile under the influence of drift and diffusion inside the blood vessel. Mobile nano-machines inside the blood vessels can be used for early detection of biomarkers released by cancer cells in the blood vessels [15]. This is also a health monitoring application.

Also, different types of MC systems have been proposed in the literature in which the simplest case considers a single transmitter and receiver. An example of a single transmitter and receiver communicating in a flow-based channel is shown in Fig. 6. A wearable device with a transmitter and receiver is implanted over human skin for monitoring the blood viscosity. The transmitter releases molecules through a needle and these molecules pass through the blood vessel. On the other hand, the statistics of the received signal are monitored at the receiver since the diffusion coefficient of the released molecules varies with viscosity of blood which in turn change the statistics of the received signal. This setup can be useful for detecting the hyper-viscosity syndrome [9].

This simple case is further extended for the MultipleInput Multiple-Output (MIMO) scenario to enhance the data rate by dividing the bit stream among multiple transmit antennas. As shown in Fig. 7, these MIMO-MC systems can also be used for improving the rate of drug delivery by using multiple transmitters and receivers. Here a transmitter (e.g., controller nano-machine) can sense the amount of drug required over the cell surface and sends a signal to the receiver for drug delivery [26]. The receiver carrying drug molecules can deliver the drug molecules at the cell surface after receiving the signaling molecules from the transmitter. This process can be faster if more than one transmitter and receiver are used for drug delivery. Further, note that in diffusion-based MC, the range of communication is limited due to the loss of molecules caused by the random Brownian motion. Therefore, to increase the range of communication, an intermediate relay node is introduced in between the transmitter and the receiver.

On the other hand, cooperative or distributed detection is proposed mostly for abnormality detection problems, where multiple receivers send their individual decision to a Fusion Center (FC) to make a global decision. 


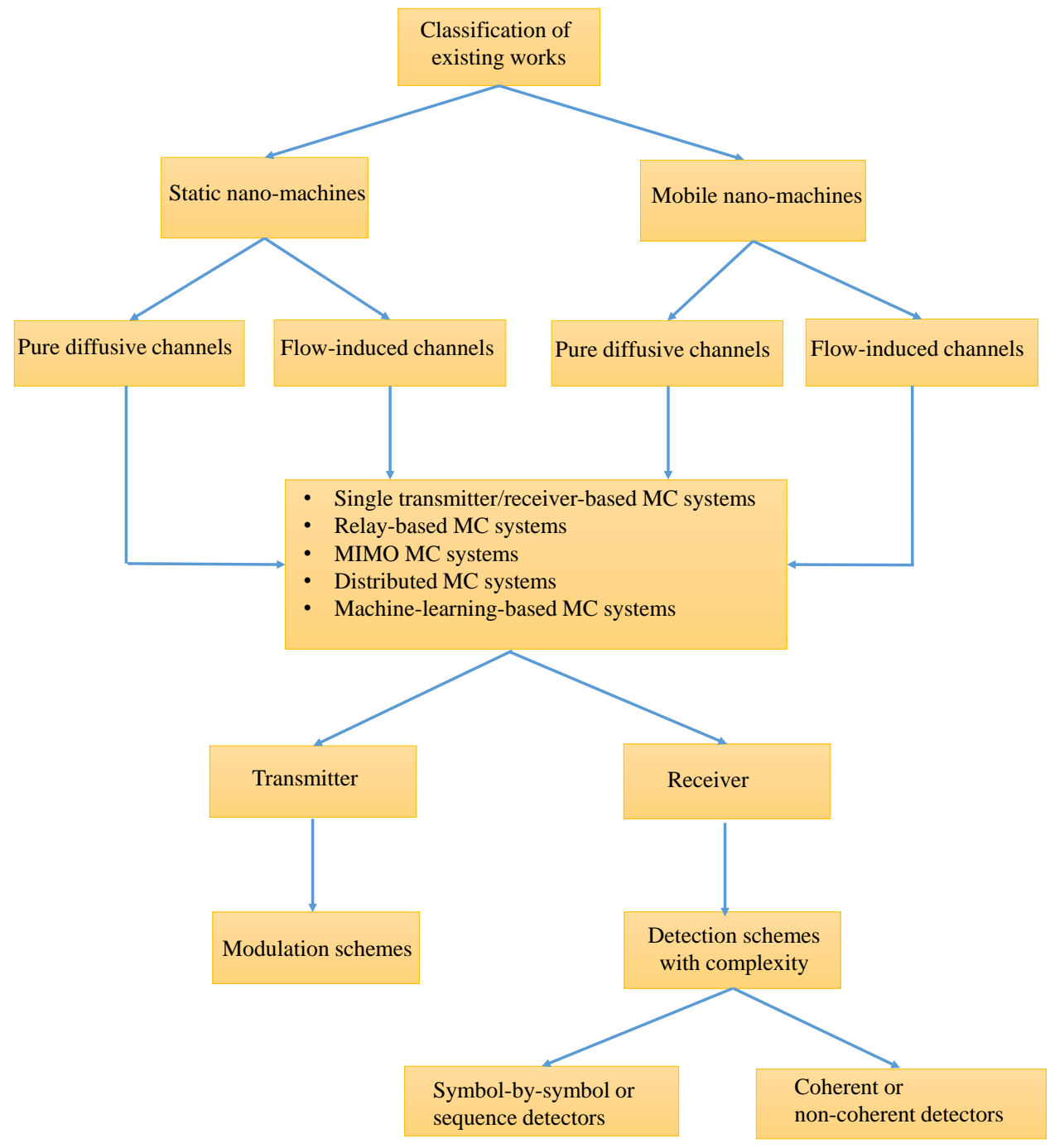

Fig. 1 - Various aspects of MC systems discussed in this paper.

An example of abnormality detection [15] has been shown in Fig. 8. This is a cooperative MC system for detecting the presence of a cancer cell. The cancer cell releases biomarkers inside the blood vessel. The concentration of biomarkers are sensed by multiple nano-sensors and finally the FC placed at a suitable location inside the blood vessel collects the reading of nano-sensors to decide whether cancer cells are present or not. For a global decision at $\mathrm{FC}$, various decision rules at FC (e.g., AND, OR, and $K$ out of $N$ rules) have been proposed in the literature. In the AND rule, the FC makes a positive decision if all the receivers send a positive decision. In the OR rule, if any one of the receivers sends a positive decision then the FC makes a positive decision. In the $K$ out of $N$ rule, the FC makes a positive decision if $K$ out of $N$ receivers send a positive decision. Note that each of the decision rules results in different system performance.
Further, various machine-learning-based MC systems [27], [28], [29], [30] are also proposed in the literature. The advantage of machine-learning-based MC systems is that the receiver does not need any channel knowledge since it can directly learn from the data [31]. These machine learning based algorithms are particularly suitable for detection in a complex environment e.g., more than two receivers in 3-D medium.

Furthermore, every MC system discussed above is intended for different applications. An example showing the possible applications of the different MC systems in IoBNT has been shown in Fig. 9. In this example, if an abnormality detection system detects an abnormality such as cancer cells, it can send molecular signals to the bio-cyber interface. The bio-cyber interface can be a wearable device that can convert molecular signals to electromagnetic (EM) signals [17] and vice versa. The bio-cyber interface further sends the signal to an access point. 


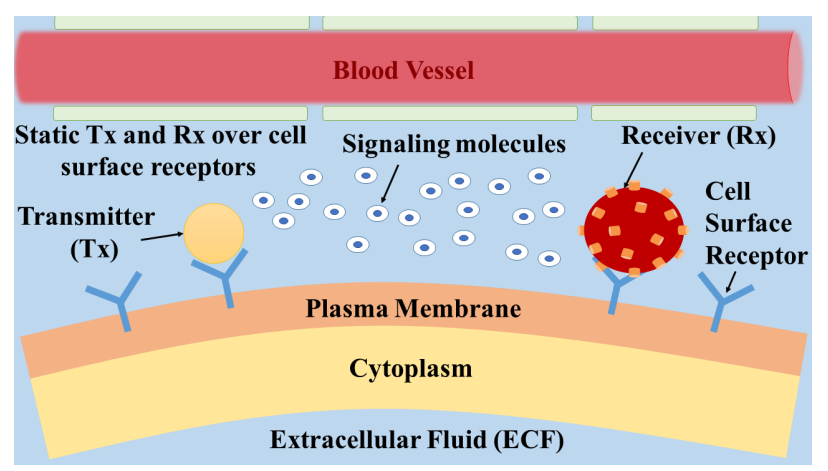

Fig. 2 - Schematic diagram of static transmitter and receiver over cell surface receptors.

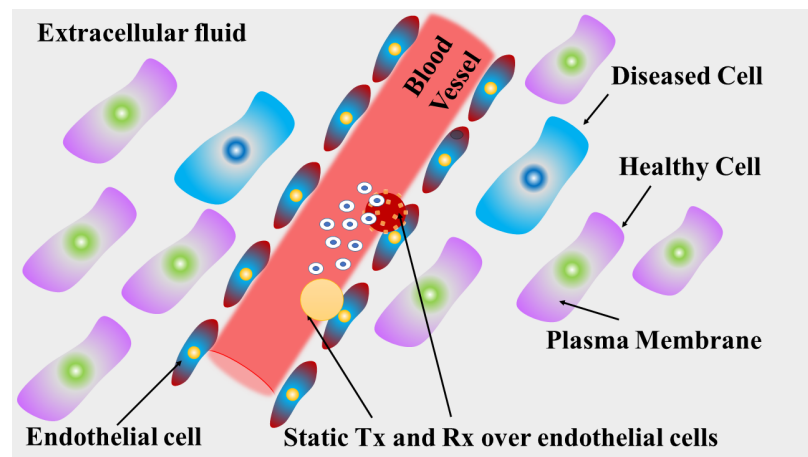

Fig. 3 - Schematic diagram of static transmitter and receiver bound to endothelial cells. Signaling molecules undergo drift and diffusion.

The signal reaches the medical personnel at a remote place through the Internet. The medical personnel can initiate the drug delivery process by sending appropriate commands to the drug delivery system inside the body. The signal propagates in the reverse order for this case. Further, different MC systems have to be used for the aforementioned scenario.

For example, cooperative MC systems are most suitable for abnormality detection and MIMO-MC systems can be used for drug delivery. The requirement of a router to send signals between different networks is also mentioned in [17]. Further, machine-learning-based MC system can be used at the FC and the bio-cyber interface as the computational complexity of machine learning algorithms may not be feasible to implement on the nanomachines with limited computational complexity. Irrespective of the complexity, this survey also covers the transmission and detection schemes proposed for all the aforementioned MC-based systems. Further, the performances and the complexities of some important detection schemes have also been discussed and compared in this survey.

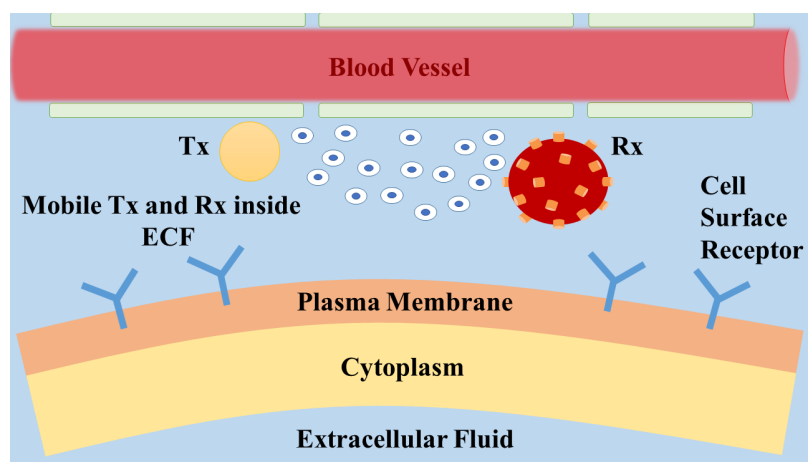

Fig. 4 - Schematic diagram of mobile transmitter and receiver inside extracellular fluid.

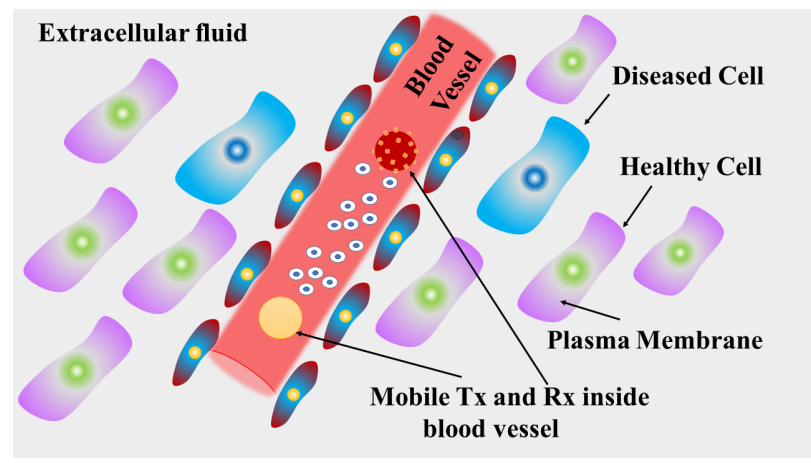

Fig. 5 - Schematic diagram of mobile transmitter and receiver inside the blood vessel. Transmitter, receiver, and signaling molecules propagate under the influence of drift and diffusion.

The rest of the paper is organized as follows: Section 2 describes the transmission and detection techniques when communicating nano-machines are static. Section 3 discusses the transmission and detection techniques for mobile nano-machines. Transmission and detection used in some of the experimental works are presented in Section 4. Challenges of practical transmitter and receiver design as well as future research directions are presented in Section 5. Finally, Section 6 concludes the paper. Also, the complete list of acronyms used in this paper is given in Table 1. 
Table 1 - List of Acronyms

\begin{tabular}{|c|c|c|c|}
\hline Acronym & Description & Acronym & Description \\
\hline Ach & Acetylcholine & MAP & Maximum A posteriori Probability \\
\hline ANN & Artificial Neural Network & MC & Molecular Communication \\
\hline ASK & Amplitude Shift Keying & MF & Matched Filter \\
\hline AWGN & Additive White Gaussian Noise & MIMO & Multiple-Input Multiple-Output \\
\hline $\mathrm{BA}$ & Biological Agent & MISO & Multiple-Input Single-Output \\
\hline BCDA & Block Coordinate Descent Algorithm & MMC & Mobile Molecular Communication \\
\hline $\mathrm{BCZ}$ & $\begin{array}{l}\text { Bovine serum albumin protein conjugated } \mathrm{ZnO} \\
\text { nano-sphere }\end{array}$ & MMSE & Minimum Mean Square Error \\
\hline BER & Bit Error Rate & MoSK & Molecule Shift Keying \\
\hline BFGS & Broyden-Fletcher-Goldfarb-Shanno & mRNA & Messenger Ribonucleic acid \\
\hline BSA & Bovine Serum Albumin & MSSK & Molecular Space Shift Keying \\
\hline CDF & Cumulative Distribution Function & MTSK & Molecule Transition Shift Keying \\
\hline CIR & Channel Impulse Response & NP & Neyman-Pearson \\
\hline CNT & Carbon Nano-Tube & OOK & On-Off keying \\
\hline CSI & Channel State Information & OSK & Order Shift Keying \\
\hline CSK & Concentration Shift Keying & PAM & Pulse Amplitude Modulation \\
\hline CTAT & Count-To-A-Threshold & PDF & Probability Density Function \\
\hline CTMP & Continuous Time Markov Process & PDMS & Polydimethylsiloxane \\
\hline DFE & Decision Feedback Equalizer & PMF & Probability Mass Function \\
\hline DFF & Decision Feedback Filter & PNN & Probabilistic Neural Network \\
\hline DNA & Deoxyribonucleic acid & PPM & Pulse Position Modulation \\
\hline ECF & Extracellular Fluid & PSO & Particle Swarm Optimization \\
\hline ECS & Extracellular Space & QMSSK & Quadrature Molecular Space Shift Keying \\
\hline EGFETs & Electrolyte-Gated Field-Effect Transistors & RF LC & Radio Frequency inductance capacitance \\
\hline FC & Fusion Center & RKHS & Reproducing Kernel Hilbert Space \\
\hline FCM & Fuzzy C-mean & RNN & Recurrent Neural Network \\
\hline FET & Field-Effect Transistor & RS & Reed-Solomon \\
\hline FRET & Forster Resonance Energy Transfer & RTSK & Release Time Shift Keying \\
\hline G-LOD & Generalized-Local Optimum Detector & SBRNN & Sliding Bidirectional Recurrent Neural Network \\
\hline G-LRT & Generalized-Likelihood Ratio Test & SEP & Symbol Error Probability \\
\hline $\mathrm{HCl}$ & Hydrochloric acid & SIMO & Single-Input Multiple-Output \\
\hline IG & Inverse Gaussian & SINR & Signal-to-Interference plus Noise Ratio \\
\hline ILI & Inter-Link Interference & Si-NW & Silicon-Nano Wire \\
\hline IoBNT & Internet-of-Bio-Nano Things & SISO & Single-Input Single-Output \\
\hline ISI & Inter-Symbol Interference & SNR & Signal-to-Noise Ratio \\
\hline LED & Light Emitting Diode & SPIONs & Super-Paramagnetic Iron Oxide Nanoparticles \\
\hline LLR & Log-Likelihood Ratio & SVM & Support Vector Machine \\
\hline LM & Levenberg-Marquardt & SWCNT & Single-Walled Carbon Nano-Tube \\
\hline LMS & Least Mean Square & TDD & Targeted Drug Delivery \\
\hline LOC & Lab-on-a-Chip & TS & Takagi-Sugeno \\
\hline LSTM & Long Short Term Memory & $\mathrm{ZF}$ & Zero Forcing \\
\hline
\end{tabular}

\section{TRANSMISSION AND DETECTION WITH STATIC NANO-MACHINES}

\subsection{Static nano-machines in pure diffusive channel}

\subsubsection{Single transmitter and single receiver- based MC systems}

In [32], authors employed an On-Off Keying (OOK) modulation scheme at the transmitter where a fixed number of molecules were transmitted for bit- 1 and no molecules were transmitted for bit-0. For this setup, optimal and suboptimal detection schemes ${ }^{1}$ were derived by maximizing the mutual information between transmitted and received symbols in the presence of ISI. In this work, perfect synchronization between the transmitter and the receiver is assumed to simplify the analysis. In contrast to OOK, the work in [33] considered a Molecule Shift Keying (MoSKmodulation scheme in which different types of molecules are sent to transmit different symbols. In this work, a linear and time-invariant model for signal propagation is assumed and the noise is modeled as the additive, uncorrelated, and non-stationary random variable with zero mean and variance dependent on the magnitude of the signal.

\footnotetext{
${ }^{1}$ In contrast to suboptimal detection scheme, optimal detection scheme requires a priori probability.
} 


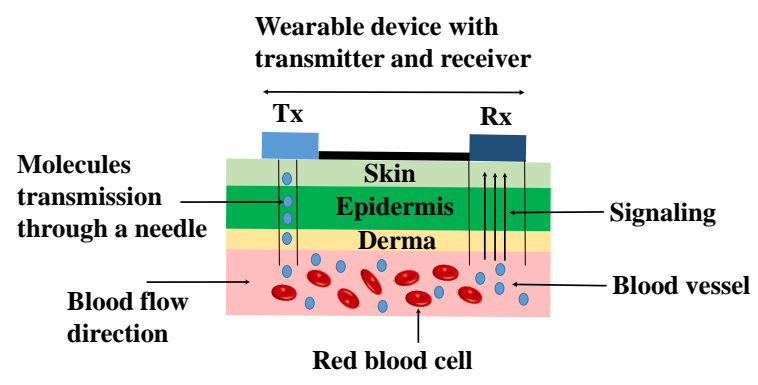

Fig. 6 - Wearable device for monitoring blood viscosity.

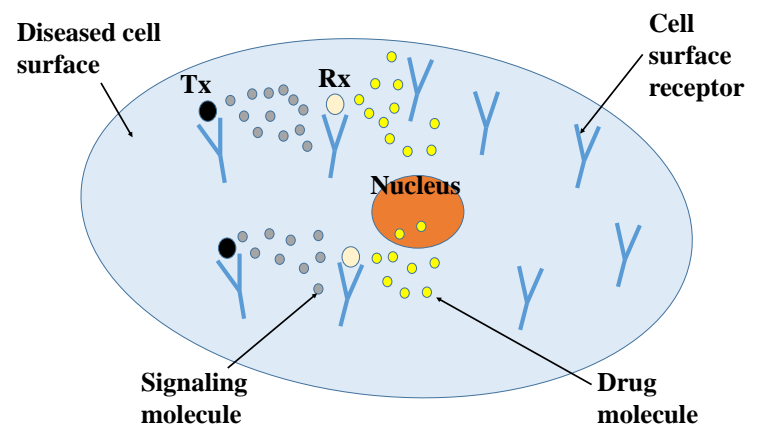

Fig. 7 - Drug delivery over a cell surface using MIMO-MC system.

A general block diagram of an MC system proposed in [33] has been shown in Fig. 10, where $y(t)$ is the received signal, $N_{\mathrm{tx}}$ is the number of transmitted molecules, $p(t)$ is the pulse shaping filter, $h(t)$ is the CIR, and $n(t)$ is the signaldependent noise (other external noise sources can also be included). The received number of molecules is assumed to be binomial which is approximated as a Gaussian distributed random variable to derive the maximum likelihood detector. In addition to the maximum likelihoodbased detector, a sequence detection scheme (i.e., Viterbi detector) is also derived since the channel with ISI adds memory to the signal which is similar to a convolutional encoder using the shift registers.

For improving the BER performance compared to [33], the authors in [34] introduced a noise whitening filter at the receiver for binary MoSK modulation-based transmission. In this work, the reception process was modeled as a Low Pass Filtering (LPF) operation where the LPF transfer function was derived using the ligand-receptor binding equation at the receiver. Moreover, the Brownian and residual noises corrupting the signal have been assumed to be signal-dependent. Finally, the system bit error rate performance is shown to improve by using a noise whitening filter.

The work in [35] derived an energy-based detector considering the binary Pulse Amplitude Modulation (PAM) based transmission using a square pulse instead of an impulse of molecules. For this setup, the mean and variance of the number of received molecules have been cal- culated by integrating the propensity function of concentration signal intensity over a symbol duration. Finally, at the receiver, the threshold-based detection was performed where the test statistic ${ }^{2}$ was shown to be composed of a constant, two Normal random variables, and a Chi-square random variable. Further, in [36], binary and 3-ary PAM transmission schemes have been used. In this work, the detection at the receiver was carried out based on the response generated by the bacteria to the input molecular signals. Four different sampling strategies of the response were studied. These strategies were based on the total response, peak response, positive and negative slopes in which the BER for positive slope sampling was found to be the worst.

In [37], the performance of NP and matched filter (MF) detectors were analyzed for the detection of a nanobiosensor signal corrupted with thermal and shot noises. It is shown that these two detectors result in identical performance in the presence of thermal noise only. However, as the shot noise increases, the NP-based test outperforms the MF detector. Also, when the binary signals to be differentiated, are very close or farther from each other, both NP and MF detectors have identical performance otherwise the NP rule outperforms. Further, binary and quaternary ASK were used as the transmission schemes in [38] and the receiver based on an NP test was proposed. In addition to this, suboptimal detection schemes with reduced complexity were also reported.

On the other hand, OOK modulation and two different methods of detection based on pulse amplitude and pulse energy with fixed threshold have been proposed in [39]. In this work, analytical expressions of communication metrics such as pulse delay (i.e., peak time), pulse amplitude (i.e., the concentration at peak time), and pulse width (i.e., the time difference between two points when concentration falls to $50 \%$ of its peak value) were derived for the pulse amplitude-based detector. Similarly, the analytical expressions of pulse energy (i.e., the integral of concentration with respect to time) and pulse duration (i.e., the time when the energy becomes a specified fraction of total energy) have been derived for a pulse energy-based detector. It is observed therein that the pulse amplitude and energy-based detectors are suitable for a high transmission rate and large transmission distance, respectively.

In [40] the transmitter used a rectangular pulse of concentration. Four different methods of detection have been proposed at the receiver. More specifically, Maximum A posteriori Probability (MAP) and maximum likelihoodbased sequence detection schemes were proposed, which maximize the joint Probability Density Function (PDF) of received samples and the transmitted bits, and use the Viterbi algorithm. Let $b_{j}$ denote the information bit trans-

\footnotetext{
${ }^{2}$ The test statistic is derived by taking the logarithm of the likelihood ratio that uses the Neyman-Pearson (NP) formula.
} 


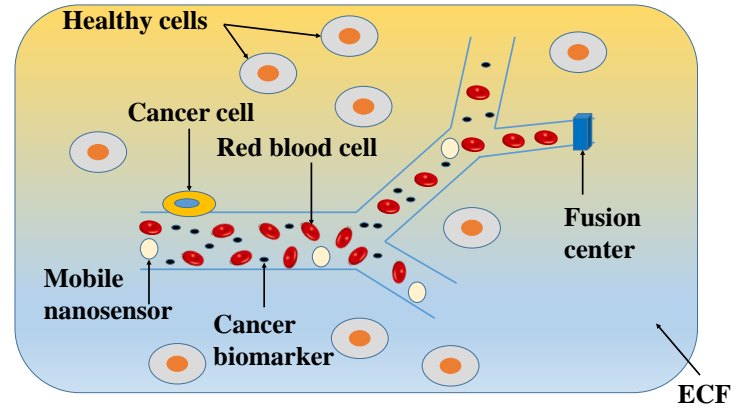

Fig. 8 - Cooperative MC system of mobile nano-sensors and fusion center using biomarker concentration inside blood vessels for detecting cancer cell.

mitted in the $j$ th bit interval. The MAP sequence detection corresponding to the transmitted bit sequence of length $N+1$ can be done according to the following equation

$$
\hat{b}_{\mathbf{0}}^{\boldsymbol{N}}=\underset{\boldsymbol{b}_{\mathbf{0}}^{\mathbf{N}}}{\operatorname{argmax}} f\left(\boldsymbol{y}_{\mathbf{0}}^{\boldsymbol{N}}, \boldsymbol{b}_{\mathbf{0}}^{\boldsymbol{N}}\right),
$$

where $\hat{b}_{0}^{N}$ represents the estimated sequence (of length $N+1)$ for the transmitted sequence $\boldsymbol{b}_{\mathbf{0}}^{\boldsymbol{N}}$, and $f\left(\boldsymbol{y}_{\mathbf{0}}^{\boldsymbol{N}}, \boldsymbol{b}_{\mathbf{0}}^{\boldsymbol{N}}\right)$ is the joint PDF of the received signal samples $y_{0}^{N}$ and the transmitted bits $\boldsymbol{b}_{\mathbf{0}}^{\boldsymbol{N}}$, which can be expressed as

$$
f\left(\boldsymbol{y}_{\mathbf{0}}^{N}, \boldsymbol{b}_{\mathbf{0}}^{\boldsymbol{N}}\right)=\prod_{k=0}^{N} \mathrm{P}\left(b_{k}\right) \prod_{k=0}^{N} f\left(y_{k} \mid \boldsymbol{b}_{\boldsymbol{k}-\boldsymbol{I}}^{\boldsymbol{k}}\right),
$$

if $b_{k}$ 's are independent, $I$ is the ISI length, $\mathrm{P}\left(b_{k}\right)$ is the PMF of the transmitted bit $b_{k}$, and $f\left(y_{k} \mid \boldsymbol{b}_{\boldsymbol{k}-\boldsymbol{I}}^{\boldsymbol{k}}\right)$ denotes the conditional PDF of $y_{k}$.

Further, in [40], a linear equalizer based on the Minimum Mean Square Error (MMSE) criterion and a nonlinear Decision Feedback Equalizer (DFE) were also proposed. Performance was evaluated for time-invariant and time-varying ${ }^{3}$ channels. It is shown that the maximum likelihood-based detector performed better than DFE, and the MMSE equalizer performed the worst among all other detectors. On the other hand, different concentration levels of molecules for binary and quaternary Amplitude Shift Keying (ASK) transmission schemes have been proposed in [41]. At the receiver, a signal detection rule based on NP tests has been derived for both schemes. Performance of both detectors was evaluated with increasing ISI and BER was found to be lower in the case of binary ASK than the quaternary ASK transmission.

A method of ISI mitigation using enzymes in the environment was presented in [24]. For system setup, OOK at the transmitter and a single sample-based detector with a fixed threshold at the receiver were employed. In this work, a lower bound on the number of received molecules

\footnotetext{
${ }^{3}$ Two different cases of time-varying diffusion coefficient were selected: i) $D(t)=2.2 \times 10^{-9}+0.8 \times 10^{-9} \cos (2 \pi t) \mathrm{m}^{2} / \mathrm{s}$, ii) $D(t)=2.2 \times 10^{-9}+$ $0.8 \times 10^{-9} \cos (10 \pi t) \mathrm{m}^{2} / \mathrm{s}$. The channel variation is 5 times faster in the latter case.
}

in the presence of enzymes was derived. Moreover, it was shown that ISI, as well as useful signals, reduce by using enzymes. For analysis purposes, the number of received molecules was shown to be binomial distributed and its approximation as Poisson distribution was shown to be more accurate than the Gaussian distribution when calculating the detection probability at the receiver.

Similar to [24], the work in [42] also used an OOK modulation scheme. However, the number of molecules released by the transmitter was modeled as a random variable whose mean was known to both transmitter and receiver. For signal detection at the receiver, a one-shot detector with three different signal processing schemes based on sampling, correlation (multiplying the received concentration by a correlation function which was constant or based on MF), and ISI cancellation (the difference between the peak concentration or concentration at symbol end time whichever is minimum and the concentration at starting time of the received concentration) were proposed. Each of the schemes results in a different test statistic. In this work, the ISI cancellation approach was found to perform better than the schemes based on sampling and correlation techniques.

In [43], a transmission scheme similar to Release Time Shift Keying (RTSK) was proposed to send a pattern of molecules based on different time-instants. For example, the pattern $\delta_{t, 1}+\delta_{t, 1.5}+3 \delta_{t, 5}$ can be used for transmission of bit-1. As shown in Fig. 11, this pattern implies that one molecule is sent at $t=1 \mathrm{~s}$ and $t=1.5 \mathrm{~s}$ timeinstant, and three molecules are sent at $t=5 \mathrm{~s}$ timeinstant. In this work, transmission, diffusion of molecules, and reception were modeled using the Continuous-Time Markov Process (CTMP). For the reception, a MAP demodulator has been employed which maximizes the posterior probability that a symbol was sent given the history of ligand-receptor complexes formed at the receiver.

Further, the bit transmission based on the release time of the molecule was proposed in [44]. In [44], three different detection schemes based on the maximum likelihood detector, linear detector, and the detector based on the first arrival were considered. The propagation time of a particle was assumed to be Lévy distributed for a purediffusive channel and inverse Gaussian (IG) for a flowinduced diffusive channel. The performance of each detection scheme was evaluated for different propagation profiles where arrival time is modeled as (i) uniformly, (ii) exponentially, (iii) IG, and (iv) Lévy distributed random variable. Further, it is shown that the detection based on the first arrival achieved the performance close to the maximum likelihood-based detection if the noise density has zero mode $\mathrm{e}^{4}$.

Further, for reducing ISI, a novel transmission scheme based on Molecule Transition Shift Keying (MTSK) was

\footnotetext{
${ }^{4}$ The value of a random variable where the PDF is maximum is defined as the mode.
} 


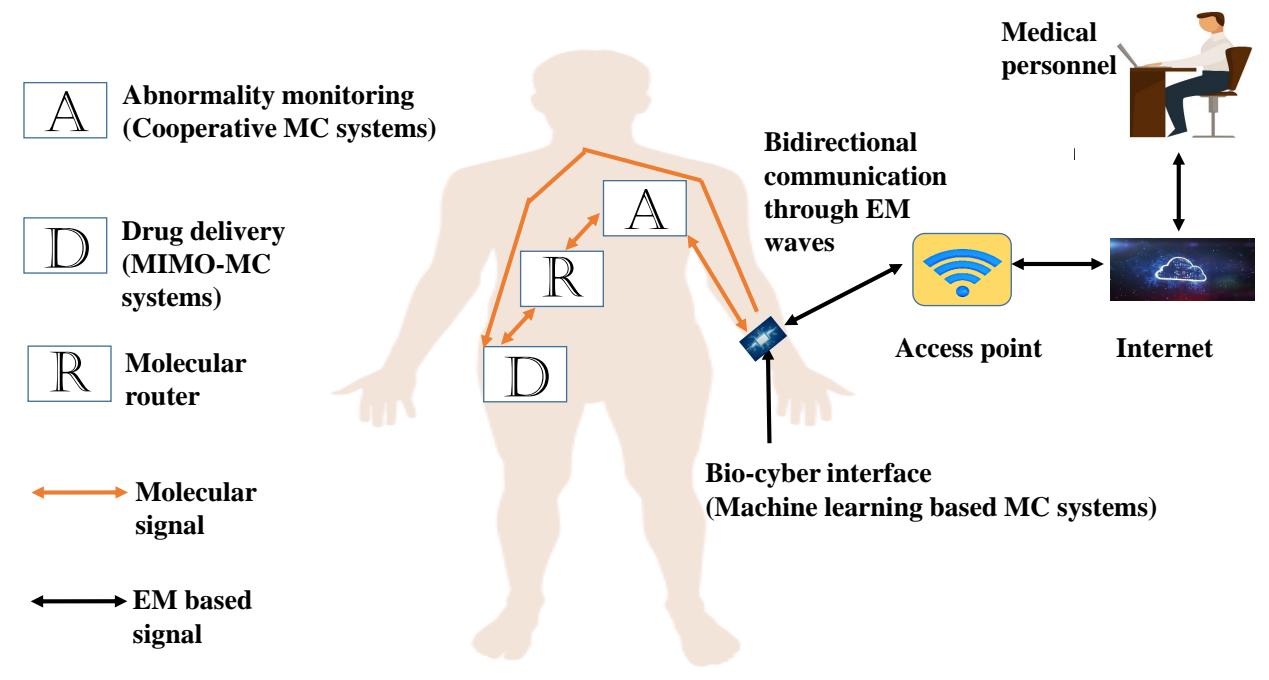

Fig. 9 - Different MC systems for different applications in IoBNT [17].

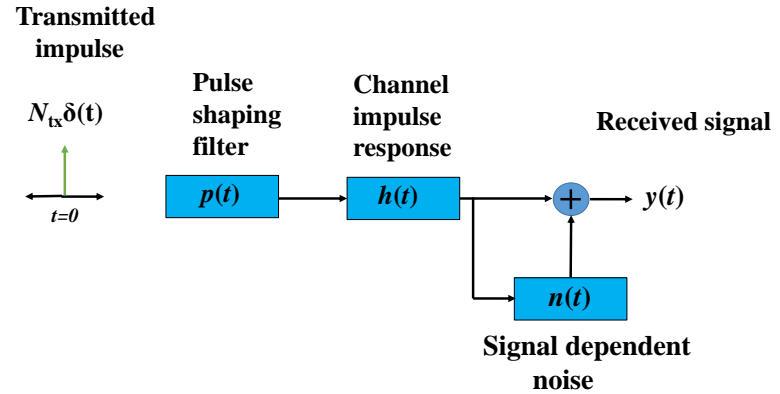

Fig. 10 - A general block diagram of an MC system.

proposed in [45], where type- $A$ and type- $B$ molecules were used for bit- 1 and no molecule for bit- 0 . As shown in Fig. 12, in contrast to the OOK modulation scheme, type- $B$ molecules were released for bit-1 before each bit-0 to reduce the effect of ISI. In addition to the MTSK modulation scheme, receiver-based ISI mitigation using a Decision Feedback Filter (DFF) was proposed where the computational complexity of DFF was independent of the number of filter taps. Further, the DFF is less complex than the conventional DFE and MMSE equalizers. The results showed that DFF significantly reduced the ISI from the received signal before comparing it with a threshold for detection. On the other hand, Impulse Transmission (IM) and pulse transmission (PAM) schemes were proposed in [46]. In this work, the energy-based detection using the binary hypothesis testing was proposed in the presence and absence of ISI. The results therein demonstrated that the IM transmission scheme has a higher probability of detection in comparison to PAM.

A low complexity adaptive threshold detection scheme has been proposed in [47] where the received signal in the current bit-interval was compared with the received signal in the previous bit-interval to decide in the favor of bit- 1 or bit- 0 , which was transmitted using the $00 \mathrm{~K}$ modulation scheme. Further, in [48], memory-less and memory-based receiver designs based on a MAP rule were proposed for 00K modulated symbols. In this work, the received signal PDF was considered as a Gaussian mixture model in presence of ISI and the detection threshold was evaluated iteratively by minimizing the probability of error. Furthermore, the authors in [49] considered rectangular pulse transmission using an OOK modulation scheme, where a non-coherent ${ }^{5}$ detection was proposed which utilized the difference between the accumulated concentration of molecules in successive bitintervals. This scheme also provides ISI mitigation capability. Further, this scheme gives better BER performance than the coherent MAP and MMSE schemes if channel memory length is restricted to 10 .

In [50], an 00K modulation scheme has been employed at the transmitter, while asynchronous peak detection and energy detection schemes with and without decision feedback were proposed at the receiver. In an asynchronous peak detector without decision feedback, all the samples within a symbol interval were compared to find the maximum value, and the maximum value was compared against the threshold for detection. Further, in the case of detection with decision feedback, the expected ISI was subtracted from the total received signal and then asynchronous peak detection was performed. A similar procedure was considered for energy detection with and without decision feedback. It is shown that the energy-based detection with ISI cancellation outperforms the other proposed schemes.

A convex optimization problem that minimizes the error probability for determining the detection threshold

\footnotetext{
${ }^{5}$ In contrast to the coherent detector, a non-coherent detector does not require Channel State Information (CSI) at the receiver nano-machine.
} 


\section{For transmission of bit-1}

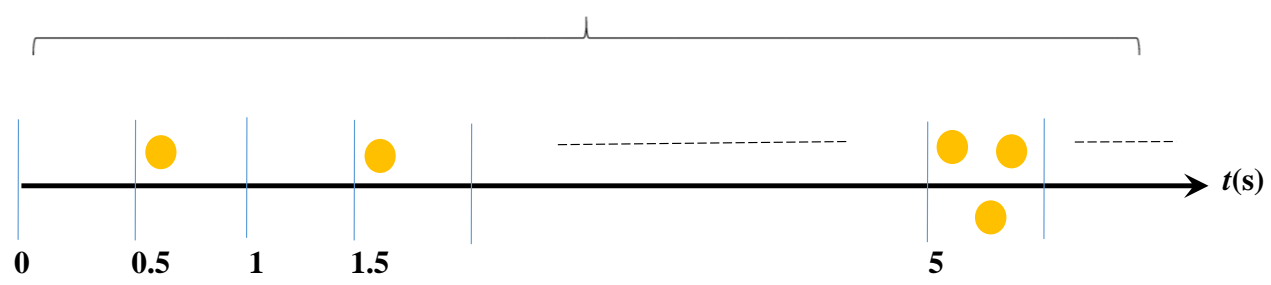

Fig. 11 - Modulation scheme similar to Release Time Shift Keying (RTSK), where the pattern $\delta_{t, 1}+\delta_{t, 1.5}+3 \delta_{t, 5}$ is used for transmission of bit-1.

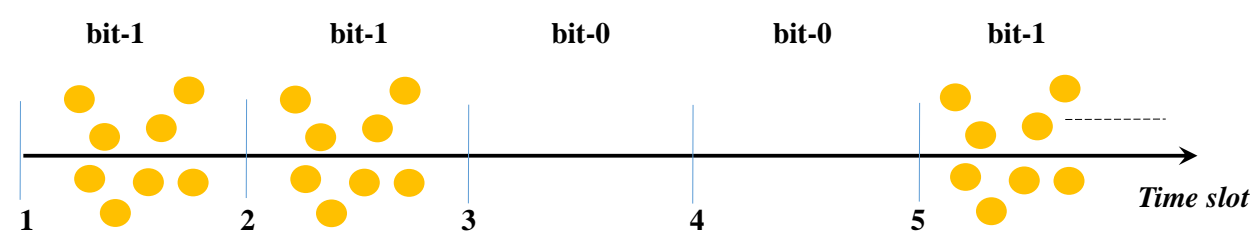

(a) Traditional OOK modulation based transmission

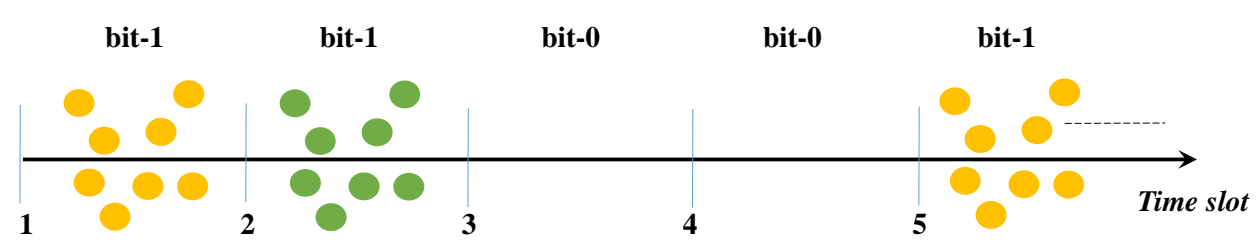

(b) MTSK modulation based transmission

Fig. 12 - MTSK modulation scheme, where two different colors represent two types of molecule.

was proposed in [51]. The logarithmic barrier function was used in optimization. Further, the Newton-Raphson method was employed for updating the threshold in each iteration. Authors in [52] proposed a non-linear and noncoherent signal detection scheme for amplitude modulated symbols, where a combination of different decision metrics was used for detection. The first decision metric was constructed using the convex property of the filtered signal, whereas the second decision metric was constructed using energy ${ }^{6}$ difference between the successive symbols. In Fig. 13, the convex nature of the signal for bit1 transmission can be observed. Further, in Fig. 14, the energy difference is positive if bit- 1 is transmitted (e.g., energy difference of 2 nd and 1st bit-intervals) and negative if bit-0 is transmitted (e.g., energy difference of 3rd and 2nd bit-intervals). Most importantly, the detection threshold at the receiver is obtained adaptively. The proposed scheme was shown to achieve BER performance

${ }^{6}$ Energy in a bit-interval is defined as the sum of all samples within a considered bit-interval.

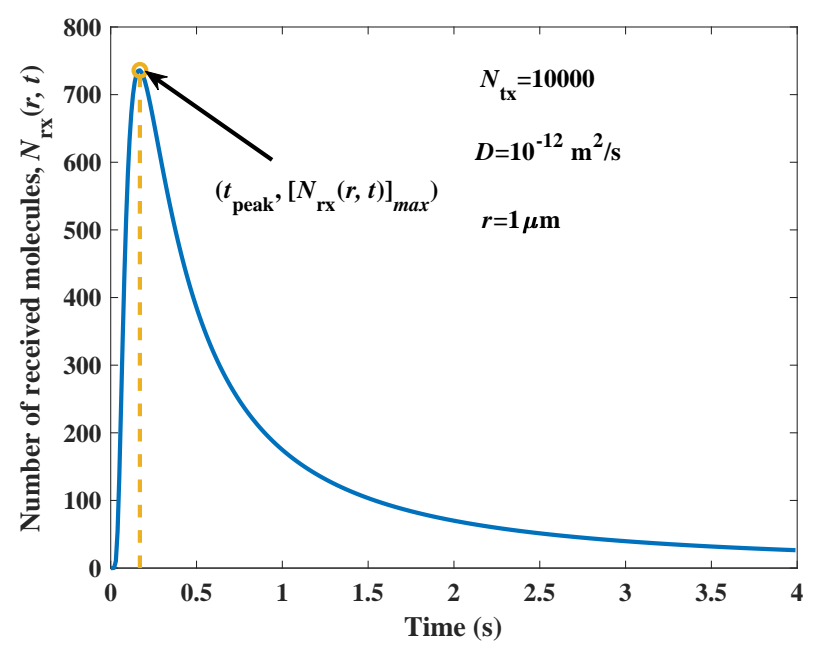

Fig. 13 - Received signal without noise considering MC-based transmission between two nano-machines. 


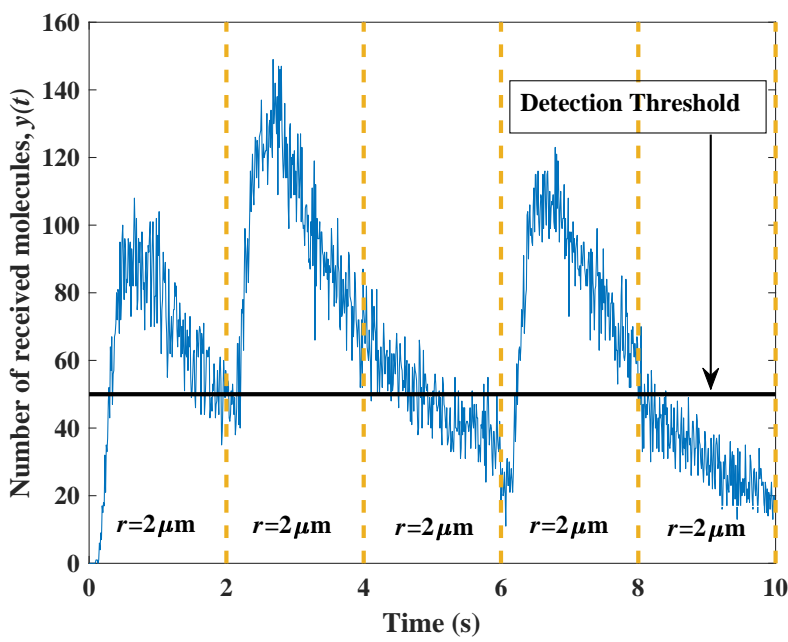

Fig. 14 - Received signal including noise and ISI for the transmitted sequence [ $\left[\begin{array}{lllll}1 & 1 & 0 & 1 & 0\end{array}\right]$. The maximum signal is above the threshold in 3rd and 5th-bit durations leading to incorrect detection.

identical to MAP and MMSE detection schemes at a significantly low Signal-to-Noise-Ratio (SNR) range.

In [53], OOK was used at the transmitter and an adaptive threshold-based detection in presence of noise and ISI was proposed at the receiver. Half of the total received molecules in the previous bit-interval was used as the threshold for detection in the current bit-interval. It is shown that the fixed threshold scheme does not work well since a varying number of molecules (depending on previous bit transmissions) due to ISI is added in the current bit-interval. A transmission scheme similar to [53] was also considered in [54]. However, at the receiver, the authors proposed energy-based and sampling-based detection schemes. For the energy-based detection scheme, an optimal time-interval was determined to minimize the error rate at the receiver. For sampling-based detection, the total number of samples and the sampling point were optimized to minimize the error rate.

The Pulse Position Modulation (PPM) scheme was proposed in [55] where molecules were released at the beginning and middle of the bit-interval in case of bit- 1 and bit- 0 , respectively. A novel low complexity MAX detection scheme was proposed where the time at which the maximum concentration occurs is determined. In the case of bit- 1 and bit- 0 , the maximum concentration occurs before and after the midpoint of the bit-interval, respectively. The performance of the MAX detector was close to the maximum likelihood detector except for scenarios that experience significant ISI. Further, similar to [55], the work in [56] also employed PPM modulation scheme at the transmitter. However, in contrast to [55], two asynchronous detectors were proposed in [56], where the first detection scheme approximated the observation vector to a linear weighted quantity and used a maximum likelihood estimate for detection. In the second method, only the first arriving molecule was considered for detection.
Thus, the first element of the observation vector was set to one, and the rest of the entries were set to zero. Based on this observation vector, a maximum likelihood estimation was carried out at the receiver.

The Reed-Solomon (RS) coding scheme for MC was introduced in [57] to enhance the error rate performance at the receiver. In this work, the system using RS coding was shown to achieve better BER than the system which uses hamming codes. The work in [58] proposed constant weight codes along with a maximum likelihood sequence detection scheme at the receiver. This detection scheme was non-coherent, i.e., CSI free detection.

A soft detection scheme using subtraction and genetic amplifier circuits modeled via chemical reactions was presented in [59]. In this work, LLR was computed assuming noise as Poisson (or Gaussian) distributed random variable. It was found that BER performance under both Poisson and Gaussian models was identical thereby justifying the Gaussian assumption. A sequence detection scheme for $00 \mathrm{~K}$ modulation has been proposed in [60]. Twolayered detection was performed where the first layer found the number of bit-1 in the received sequence of 7 bits. A different group of 7-bit patterns was divided into zones based on a logarithmic metric. Zones containing more number of bit- 1 had a higher metric. Then the second layer found the locations of bit- 1 in a specific zone by using a Pearson correlation metric. This process need not search all the 7-bit states thereby reducing the complexity.

On the other hand, a detector based on the derivative of the received signal considering the 00K-based transmission was proposed in [61]. More specifically, for detection of bit $b_{j}$ at the receiver, all the derivative values within $j$ th time slot of duration $T$ were calculated using the received signal $y(t)$, and then the maximum of these derivative values was compared against the fixed threshold $\omega$ as shown below.

$$
\hat{b}_{j}= \begin{cases}1 & \text { if } \max \frac{y\left(k T_{s}\right)-y\left((k-1) T_{s}\right)}{T_{s}} \geq \omega, \\ 0 & \text { otherwise }\end{cases}
$$

where $j N_{s} \leq k<(j+1) N_{s}$, sampling time $T_{s}$ is defined as $T_{s}=T / N_{s}$ and $N_{s}$ is the number of samples taken in a bit interval. Note that the threshold $\omega$ can be determined empirically, where minimum BER is achieved. Simulation results showed that the proposed detector performed better than the MAP detector at a high data rate or small bit duration.

Further, a preprocessing scheme based on higher order derivatives has been proposed in [62]. Note that higher order derivatives can be used if a high transmission rate is required compared to the first order derivative used in [61]. This is because of the fact that the peak time reduces with an increase in the derivative order. Also, higher order derivative processing offers better ISI mitigation since 
the amplitude of the signal reduces quickly. However, higher order derivatives may amplify the noise. Hence the optimal derivative order is required for a trade-off between the noise amplification and ISI mitigation. For decoding, maximum likelihood sequence detection with reduced channel memory was used in [62].

In [63], a variant of OOK was proposed in which two different types of molecules were considered for transmission of bit-1, and no molecules were released for bit- 0 . For bit-1, the type- $A$ molecule was transmitted at the beginning of a bit-interval, and the type- $B$ molecule was transmitted at the peak time of the type- $A$ molecule. Further, in [63], another modulation scheme was the Order Shift Keying (OSK) in which type- $A$ molecule was transmitted at the beginning of a bit-interval, and the type$B$ molecule was transmitted at the peak time of type- $A$ molecule for bit-1 and the order of molecules transmission was reversed for sending bit- 0 . These procedures reduced the ISI at the receiving nano-machine as type$A$ and type- $B$ molecules were assumed to react together. The schemes proposed are shown in Fig. 15. In addition to these schemes, a suboptimal maximum likelihood detection scheme and ISI neglecting detection scheme were also proposed.

The authors in [64] proposed an optimal detection scheme based on Accelerated-Particle Swarm Optimization (A-PSO). This scheme used an acceleration factor to find the optimal weights $\boldsymbol{w}=\left[w_{j N_{s}}, w_{j N_{s}+1}, \cdots, w_{(j+1) N_{s}}\right]$, such that the error probability is minimum as described below.

$$
\boldsymbol{w}=\underset{\boldsymbol{w}}{\operatorname{argmin}} P_{e} .
$$

Finally, using these optimal weights, a weighted sum detector was employed at the receiver i.e., each sample within a bit-interval was multiplied by a weight and all the weighted samples were added to compare against the threshold $\theta$ for detection,

$$
\hat{b}_{j}= \begin{cases}1 & \text { if } \sum_{k=j N_{s}}^{(j+1) N_{s}} w_{k} y_{k} \geq \theta ; \\ 0 & \text { otherwise }\end{cases}
$$

where $w_{k} \geq 0$. This detector was shown to perform better than the MF detector.

Further, a suboptimal detection scheme was presented in [65], where the CSI was modeled as a Gamma distributed random variable. A non-coherent decision feedback detection was also proposed therein, which utilizes statistical CSI. The proposed decision feedback detector considered a fixed detection window of size $K$ where a constant CSI was assumed. Further, a blind CSI estimation-based detection was also proposed in which CSI estimates were found by averaging over the expected positions of bit-1 and bit- 0 .

In [66], two different detection schemes based on the maximum likelihood criterion were presented for a ligand-receptor type communication. The first technique considered the likelihood of observing bound receptor molecules given the current transmitted bit and the estimate of ISI bits. The other one considered the likelihood of observing the unbound time of receptors for detection. The latter technique experienced better BER performance because the bound state of receptors can provide little information when the receiver saturates due to the ISI.

Further, three different detection techniques based on the number of bound receptors, unbound time (around the sampling time) of the receptors, and bound time of the receptors were proposed in [67]. The detection based on the bound time of the receptors was better in performance than the other two schemes. In this work, an estimator based on the log-likelihood of the ratio of ligand and total concentration was also proposed for the detection. In [68], chemical reaction networks for detecting binary MoSK signals were proposed. In this work, sampling was based on transcription networks and the demodulation process used an integral feedback controller that senses whether a chemical signal is above the threshold or not. The demodulator output is used for detecting symbols using the biological XOR gate.

In [69], OOK with rectangular pulse shaping was used at the transmitter. The transmitted pulse was convolved with the CIR to obtain the received signal. Further, at the receiver, the detection process was carried out in three steps: (i) stochastic resonance-based nonlinear filtering (processing based on local transient features), (ii) calculation of the non-coherent metric, and (iii) threshold-based detection. The decision metric was constructed by adding the metrics of the convex property of the filtered signal, transient shape among the symbols, and the energy difference between the successive symbols.

The derivation of an event (e.g. presence of a cancer cell) detection probability was presented in [70], where the event was defined as the hitting of a single molecule at any one of the multiple receivers within a certain period. The detection probabilities were derived for both degradable and non-degradable molecules. In this work, the centers of the receivers were assumed to be distributed as a Poisson point process. Also, the number of molecules to be transmitted for a specified probability of event detection was derived.

Rectangular pulse-based 00K was used as the modulation in [71]. Further, a weighted sum method with optimal weights was proposed for detection and ISI mitigation at the receiver. Optimal weights were obtained by maximizing the Signal-to-Interference-plus-Noise Ratio (SINR) i.e., differentiating SINR with respect to weights and setting it equal to zero. Also, a block-wise data detection-based iterative method for ISI mitigation was proposed where the expected ISI was subtracted from the total received signal. Simulation results showed that the iterative method outperformed the weighted sum method for ISI mitigation. 

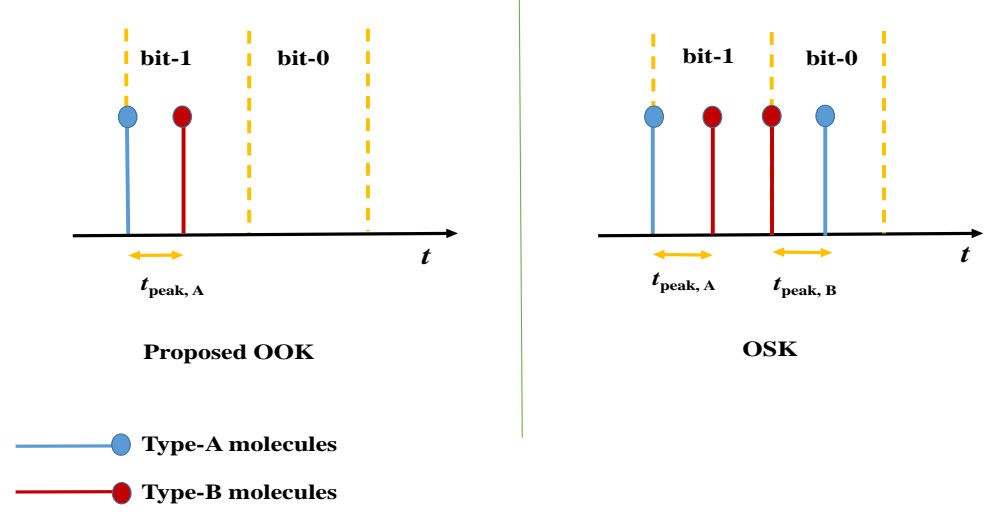

Fig. 15 - A variant of OOK and OSK modulation schemes.

For better ISI mitigation in comparison with [71], a scheme based on non-linear mapping was proposed in [72]. This scheme first calculated the peak time of the CIR and then the CIR before peak time was multiplied by a factor such that the peak value of the CIR should increase. Also, for ISI mitigation, the CIR after peak time was multiplied by a different factor to reduce the CIR tail. Further, for 00K-based transmission, a threshold-based detection was performed using signal energy as a decision metric. In this case, the threshold at the receiver was selected as the mean of the signal energies of the previous $P$ bit-intervals. Note that this selection of threshold is valid only if $P$ is significantly large. Simulation results demonstrated that this non-linear mapping scheme had better BER performance compared to the scheme proposed in [71].

In [73] CSK modulation similar to [74] was used at the transmitter. The detection was done by maximizing the Probability Mass Function (PMF) of the number of received molecules. A similar detection procedure was also presented considering the presence of an additional source. This additional source generated interference which was modeled using a uniformly distributed random variable for mathematical analysis. Also, an optimal detection interval was found, which minimized the error probability. It was shown that the optimal detection interval was less than the transmission bit-interval. Authors in [75] proposed coded modulation for binary OOK and 4-ary CSK. Moreover, at the receiver, threshold-less detection of data symbols was proposed since the threshold was set to zero due to the orthogonal coding scheme. However, the data rate was limited ${ }^{7}$ for the proposed system due to channel coding.

Further, a generalized MoSK modulation was proposed in [76] to improve the data rate using less types of molecules

\footnotetext{
${ }^{7}$ Since the channel coding schemes append some coded bits to the information bits, the amount of information transmitted in a given time is reduced in comparison to the scenario which does not consider channel coding.
}

compared to the conventional MoSK modulation [33]. The generalized MoSK can transmit $\left\lfloor\log _{2}\left(M ! /\left(M_{I} !(M-\right.\right.\right.$ $\left.\left.\left.M_{I} !\right)\right)\right\rfloor$ bits per symbol where $M_{I}$ out of $M$ types of molecules were required to send a symbol. While the MoSK scheme can only transmit $\left\lfloor\log _{2}(M)\right\rfloor$ bits per symbol using $M$ types of molecules. For example, $M=16$ is required in MoSK while $M=6$ and $M_{I}=3$ is required in generalized MoSK to achieve a data rate of 4 bits per symbol. Apart from this, maximum likelihood detection considering the presence/absence of ISI from previous one symbol duration was used at the receiver. The simulation results demonstrated that the proposed modulation scheme with a maximum likelihood detector achieves better error performance compared to MoSK, CSK and D-MoSK schemes.

\subsubsection{Relay-assisted-based MC systems}

The work in [74] proposed CSK modulation where $Q_{1}$ molecules were emitted to transmit bit- 1 and $Q_{0}\left(<Q_{1}\right)$ molecules were emitted to transmit bit- 0 . This reduces the Peak-to-Average-Molecule Ratio that is suitable for nano-machines having limited storage. Further, an estimate-and-forward relaying scheme was proposed, which was shown to perform better than the decode-andforward, and amplify-and-forward relaying schemes. In contrast to decode-and-forward relaying, this improvement arises since the estimate-and-forward relay transmits a soft decision. Also, the estimate-and-forward relay does not amplify the noise as seen in the amplify-andforward relaying scheme. The maximum likelihood estimation of the number of transmitted molecules was performed at the intermediate relay node which was also used to find the error probability at the destination receiver. The optimization of the decision threshold in [74] was similar to the one proposed in [51].

The work in [77] considered an amplify-and-forward relaying scheme to increase the communication range. In this work, a detection scheme based on the MAP rule has been proposed for OOK modulated transmission. The detection threshold was determined by differentiating the 
expected error probability with respect to the threshold and substituting the resulting expression equal to zero. Further, in [78], equal gain combining was used at the final destination which could sense the molecules sent by both the transmitter and the decode-and-forward relay node. In this work, the optimal position of the relay and optimal detection threshold at each of the receivers were derived by using a Block Coordinate Descent Algorithm (BCDA) which minimizes the end-to-end probability of error. Note that single type of molecules transmission at both the nodes causes ILI that in turn decreases the channel capacity. Thus, to improve the channel capacity of a decode-and-forward relay-assisted-based MC system, different types of molecules were used for transmission at the source and the relay in [79]. For decoding, a joint optimal detection threshold problem was also solved using the Gradient Descent algorithm, which minimizes the error probability with respect to thresholds at the intermediate relay and the final destination.

\subsubsection{MIMO-based MC systems}

The work in [80] proposed a binary ASK modulation scheme for the MIMO-MC system. For the proposed system, a Zero-Forcing (ZF)-based equalizer, as well as fixed and adaptive threshold-based detection schemes, have been proposed. Simulation results demonstrated that both ZF and adaptive threshold-based detectors had identical BER performance, however, the fixed thresholdbased detection experienced the worst BER performance at the receiver.

Derivative-based detection for $2 \times 2$ MIMO-MC considering $00 \mathrm{~K}$ modulation was proposed in [81]. In this work, the detection was based on the premise that when a bit- 1 was transmitted the received signal had consecutive positive derivative values due to an initial rise in the number of received molecules, and in the case of bit- 0 , the received signal either had consecutive negative slope values or small positive values due to a weak rising edge caused by the Inter-Link Interference (ILI). Further, for detection in a severe ILI scenario, a ZF detection scheme was proposed in [82] assuming asymmetric topology (different number of transmit/receive antennas). The received signal was multiplied by the Moore-Penrose pseudo inverse of the channel matrix to counter the ILI and also to yield an estimate of the transmitted signal. Simulation results showed that ZF detection with signal sampling at peak times of respective receive antennas achieved lower BER than the ZF detection with signal sampling at the same time for all receive antennas.

A MIMO-MC system where a transmitter could send different types of molecules was proposed in [83]. In this work, optimizing the drug dosage (i.e., number of molecules emitted by the transmitter) was considered. For performance analysis, BER was evaluated for four different configurations i.e., Single-Input Single-Output (SISO), SingleInput Multiple-Output (SIMO), Multiple-Input Single Out- put (MISO), and MIMO. Further, the work in [84] derived approximate analytical expression of hitting probability considering the the presence of two fully absorbing receivers in 3-D medium. Based on this expression, authors demonstrated that a signaling molecule has higher hitting probability if two fully absorbing receivers at a distant location are used instead of a single fully absorbing receiver. Further, considering null and alternative hypotheses with OOK modulated symbols, the area under the ROC curve was derived to analyze the detection performance at both receivers. The insights developed in this work can be further used for designing the detection schemes with two receivers.

\subsubsection{Distributed detection-based MC systems}

The work in [85] considered cooperative detection where more than one receiver detects the 00K modulated transmitted bit and send their decision to an FC, which makes a global decision using the majority, AND, and OR rules. Simulation results demonstrated that the error probability for the majority rule was the lowest. In the noisy scenario, the OR rule performed better for high threshold values. A square pulse transmission based on $00 \mathrm{~K}$ was proposed in [86] for abnormality detection. In this work, the abnormality detection scheme considered a two-tier structure, where several sensor nano-machines sensed the abnormality and sent their decisions to an FC to decide the presence or absence of abnormality. The NP criterionbased generalized likelihood ratio test was used for detection in the first tier and the OR-based fusion rule [85] was used at FC in the second tier.

Authors in [87] formulated a convex optimization problem to determine the threshold at each of the receivers and FC, which minimizes the expected end-to-end probability of error. The performance of the system was evaluated under perfect and noisy reporting by the receivers to the FC. A similar system of sensor nano-machines sending molecular signals to an FC for cooperative abnormality detection was proposed in [88]. In this work, two different transmission schemes emitting the same type of molecules and different types of molecules by sensors to the FC were considered. For detection, LLR was compared against the threshold to decide whether an abnormality is present or not. In addition to this, suboptimal detection schemes for different types of molecules were proposed, where only the maximum value inside the logarithm function was considered. Moreover, the maximal ratio combining of the sensor outputs and two-stage detection were also proposed therein.

Further, to reduce the number of samples and subsequently decision delay at the FC, a detection technique was proposed in [89]. This technique used a sequential average probability ratio test, which employed two different thresholds for binary hypothesis testing. A decision was made in favor of the first hypothesis if LLR was less than the lower threshold otherwise the second hypothesis 
was chosen if LLR was greater than the upper threshold. If the LLR was in between the lower and the upper thresholds, then only one more sample was collected and LLRT was repeated again. A communication system similar to [85] was proposed in [90]. In this case, multiple receivers emitted the same type of molecules to convey information to the FC and at the FC, a simple detection based on a constant threshold was proposed for decoding information bits. This scheme was slightly inferior to the majority rule considered in [85], which however had low complexity.

A communication system based on several nano-sensors sending information to the FC was proposed in [91]. This system was for detecting the presence or absence of target (malignant tissue) considering the unknown secretion rate of biomarkers and unknown location of the malignant tissue. In this work, the LLR test was used at the nano-sensors for detection, whereas the GeneralizedLikelihood Ratio Test (G-LRT) and Generalized-Local Optimum Detector (G-LOD) were proposed at the FC. In contrast to G-LRT, G-LOD was less computationally complex since it maximized the decision variable with respect to target location only. A MIMO communication system was proposed in [92] where multiple transmitters sent different molecules to the receivers. A signal processing scheme to find the global peak out of various impulse responses received at a single receiver was presented. Further, an NP test was performed at each of the receivers to decide in favor of bit-1 or bit-0. Finally, the central node i.e., FC collected all the individual decisions from each receiver and made the decision using $K$ out of $N$ rule i.e., if $K$ or more than $K$ receivers decide bit- 1 then FC decides bit-1.

On the other hand, three different variations of maximum likelihood detection were proposed in [93]. In a perfect reporting scenario: i) the $\mathrm{FC}$ assesses each observation by the receiver to calculate the likelihood. This is termed as Full-maximum likelihood. ii) The FC assigns equal weight to all the samples within a bit-interval at each receiver. This is Limited-maximum likelihood detection. In noisy reporting, the FC adds all the observations that arrive from different receivers and finds the likelihood. All the receiver's use single molecule and decode-andforward strategy to send information to the FC. Hence this technique was termed as SD-maximum likelihood. SDmaximum likelihood had the worst performance and the Full-maximum likelihood detection performed the best.

Abnormality detection and monitoring schemes using sensor networks with FC have been presented in [94]. Sensors used the 00K modulation scheme to transmit information to the FC. After collecting observations from all the sensors, the FC makes a final decision on the state of abnormality. Note that this system was based on a partially observable Markov decision process (nonhomogeneous Markov model). In this model, the system state was defined as the location and time of occurrences of abnormalities at multiple sites, and how they propa- gate. Different situations in the system were mapped to some states of the Markov process. Further, the probability for each state was calculated and updated. Based on these probabilities, the changes in the system were monitored. More specifically, the spreading of changes in the environment (e.g. gradual tumor growth) was studied for monitoring. On the other hand, suboptimal detectors based on myopic policy were proposed. For detection, the aim was to minimize the delay in reporting to the FC. Furthermore, binary and non-binary stopping time scenarios were investigated.

In [95], two maximum likelihood detection schemes (single molecule type and multiple molecule types) at FC based on decode-and-forward relaying and an maximum likelihood detection scheme based on amplify-andforward relaying was proposed. It was shown that the multiple molecule type scheme outperformed the other two proposed schemes for the considered cooperative MC system.

\subsubsection{Machine-learning-based MC systems}

An adaptive threshold-based detection using an Artificial Neural Network (ANN) was proposed in [27]. In this work, the Gradient-Descent algorithm was used for weight optimization. Moreover, elements of the output vector of the neural network were used as an adaptive threshold for detecting the bits, e.g., if the number of received molecules in $j$ th bit duration was $N_{\mathrm{rx}_{j}}$ and $o_{j}$ was the corresponding output of ANN then $N_{\mathrm{rx}_{j}}>o_{j}$ was the rule for detecting bit-1 and vice-versa. In this work, BER performance better than state-of-the-art detectors was claimed at a reduced number of transmitted molecules.

In [96], curve fitting for the received molecules is done, where the Least Mean Square (LMS) method is used to update the polynomial coefficients. Moreover, the weighted sum of polynomial coefficients is used as the adaptive threshold for detecting the information in the presence of ISI. Further, Takagi-Sugeno (TS) fuzzy model has been employed, which can approximate complex nonlinear systems with fewer rules and higher modeling accuracy, and express the local dynamics of each fuzzy rule. The TS model, which is comprised of an optimized structure (the number of rules and inputs), optimized parameters for membership function. This strategy can minimize the error between the fuzzy output and the desired output. This work also proposed an ANN detector with two hidden layers, which combines the adaptive fuzzy threshold (in the first hidden layer) and polynomial approximation (in the second hidden layer).

Further, for a CSK-based modulation scheme, a deep learning-based detector was proposed in [28] that minimized the difference between detected and transmitted symbol vectors. The detector had 70 neurons in the first hidden layer and 10 neurons in the second hidden layer. This detector performed well at SNR values of $18 \mathrm{~dB}$ and 
$15 \mathrm{~dB}$ for ISI and ISI free scenarios respectively. Furthermore, to reduce the number of hidden layer neurons, a different architecture of the feed-forward ANN detector has been proposed in [29] where the training of a neural network was carried out for zero-bit, one-bit, and $K$-bit memory receivers using the number of received particles and the corresponding transmitted bit. The number of hidden layers was 10 with 5 neurons in each layer. Moreover, the Levenberg-Marquardt (LM) optimization algorithm has been used for updating the weights. The detection was based on whether the probability of observing a bit-1 given the number of received particles and previously estimated bits was greater or less than 0.5 .

Further, two different detectors using feed-forward NN having one hidden layer of 16 nodes and RNN having one Long Short-Term Memory (LSTM) layer of 16 nodes at a fusion center in a distributed detection system was proposed in [97]. This system was proposed for a binary hypothesis testing to detect the presence or absence of an abnormality considering 00K-based transmission. These detectors were trained using the received signal at the FC from all the sensors and the corresponding hypothesis. Adam optimizer and Gradient Descent with momentum were used for feed-forward NN and RNN, respectively. These schemes avoid the requirement of an analytical channel model and CSI estimation particularly for distributed MC systems. It was shown that an RNN detector performed well than the feed-forward NN. Table 2 summarizes the modulation and detection techniques in static MC without drift in the channel.

\subsection{Static nano-machines in flow-induced diffusive channel}

\subsubsection{Single transmitter and single receiver- based MC systems}

In [98], a MAP rule-based signal detection scheme with ISI cancellation was presented for binary and M-ary transmissions. The ISI cancellation was achieved by subtracting the expected ISI molecules from the number of received molecules in the current bit-interval. In this work, positive drift velocity has been assumed and the PDF of first hitting time of a molecule is considered as IG. The maximum likelihood sequence detection along with two variations of a weighted sum detector i.e., equal weight detector and MF detector were proposed in [99] for different types of flow present in the environment. It is shown that the maximum likelihood sequence detection outperformed the other two detectors for most of the scenarios. However, both weighted detectors performed well if a mild flow was present opposite to the direction of information flow. Further, the work in [105] considered OOK modulation and proposed an MF detector which maximizes the SINR to calculate the optimal weights to be multiplied by each sample within a bit-interval. However, the selection of the detection threshold was not specified therein.
In [112], binary and 4-ary amplitude modulation schemes have been presented. Apart from these two schemes, authors also proposed a third modulation scheme where symbols 01 and 10 were considered as 1 , and 00 and 11 were considered as 0 and 2, respectively. For each scheme, Symbol Error Probability (SEP) expression was derived considering maximum likelihood detection at the receiver. Authors demonstrated that in positive drift scenario, SEP for all three schemes decreased with an increase in flow velocity. Moreover, SEP performance of the third modulation scheme was in between the other two modulation schemes. Extending the previous work [99], the authors in [113] proposed Viterbi sequence detection to obtain a lower bound on the BER. Further, a family of weighted sum detectors (i.e., MF and equal weight detectors) were also proposed and their BER performances were compared with the Viterbi detector. Analytical expressions were also derived for different weighted sum detectors. Further, ISI mitigation by using the enzymes in the environment was also included to enhance the performance at the receiver.

In [100], an 00K modulation scheme was considered for transmission. However, for detection, the variance of the propagation time of molecules was calculated and subtracted from the current time to find the emitting time of the molecules. This detection process was asynchronous. In this work, the PDF of propagation time for superior vena cavae at $120 \mathrm{~mm} / \mathrm{s}$ blood flow velocity and capillaries at $790 \mu \mathrm{m}$ were shown. Further, for performance analysis, the error probability expression in terms of the Chi-square Cumulative Distribution Function (CDF) is derived. Finally, the authors showed that the variance of propagation time PDF was lesser in superior vena cavae than the capillaries. Therefore, the communication in superior vena cavae was slightly more reliable than in capillaries. In [101], MoSK modulation was used for transmission where $N$ molecules of type- $A$ and type- $B$ were released for sending bit- 1 and bit-0, respectively. A novel asynchronous detector named Count-To-AThreshold (CTAT) was designed which counted both types of molecules and then decided in favor of a particular bit if the number of molecules corresponding to that bit was above a predefined threshold. In the presence of ISI, this detector resulted in a better performance than the simple binary detector, which compared the number of type- $A$ received molecules with type- $B$ received molecules.

The work in [103] proposed a binary timing-based modulation scheme for conveying the information between two communicating nodes. Also, three different detection schemes were proposed at the receiver. The first detection scheme was based on MAP criteria where a conditional PDF of output was maximized for detection; however, it was very complex as the multiplication of several IG distributions were required. The second technique was 
Table 2 - Summary of transmission and detection in static MC without drift

\begin{tabular}{|c|c|c|c|c|c|}
\hline Reference & Modulation & Detection & $\begin{array}{l}\text { Symbol- } \\
\text { by-symbol } \\
\text { (Sbs)/Sequence } \\
\text { (Seq) detector }\end{array}$ & $\begin{array}{l}\text { Coherent/ } \\
\text { Non-coherent } \\
\text { detection }\end{array}$ & Complexity \\
\hline [33] & MoSK & $\begin{array}{l}\text { i) MAP detection without ISI } \\
\text { ii) Viterbi detection with ISI }\end{array}$ & $\begin{array}{l}\text { Sbs for i) and } \\
\text { Seq for ii) }\end{array}$ & Coherent & $\begin{array}{l}\text { i) High } \\
\text { ii) Very high }\end{array}$ \\
\hline [35] & Binary PAM & Log LRT for energy detection scheme & Sbs & Coherent & High \\
\hline [37] & OOK & $\begin{array}{l}\text { NP test (for separating very close bi- } \\
\text { nary signals) and MF detector }\end{array}$ & Sbs & Coherent & Low \\
\hline [39] & OOK & $\begin{array}{l}\text { Amplitude and energy detection with } \\
\text { fixed threshold }\end{array}$ & Sbs & Non-coherent & Low \\
\hline [34] & MoSK & $\begin{array}{l}\text { MAP detection with noise whitening } \\
\text { filter }\end{array}$ & Sbs & Coherent & High \\
\hline [40] & $\begin{array}{l}\text { Rectangular pulse } \\
\text { based OOK }\end{array}$ & $\begin{array}{l}\text { Adaptive receiver based on steepest } \\
\text { descent algorithm for i) MAP detec- } \\
\text { tion ii) Maximum likelihood detection } \\
\text { iii) MMSE equalizer iv) DFE }\end{array}$ & $\begin{array}{l}\text { Seq for i), ii) and } \\
\text { Sbs for iii), iv) }\end{array}$ & Coherent & $\begin{array}{l}\text { i) Very high } \\
\text { ii) High } \\
\text { iii) Low } \\
\text { iv) Moderate }\end{array}$ \\
\hline [41] & $\begin{array}{l}\text { Binary and quater- } \\
\text { nary ASK }\end{array}$ & $\begin{array}{l}\text { Log LRT for binary and M-ary detec- } \\
\text { tion }\end{array}$ & Sbs & Coherent & High \\
\hline [43] & $\begin{array}{l}\text { Modulation based } \\
\text { on chemical reac- } \\
\text { tion rate }\end{array}$ & MAP rule using CTMP & Sbs & Coherent & Moderate \\
\hline [45] & MTSK & DFF & Sbs & Coherent & High \\
\hline [86] & OOK & $\begin{array}{l}\text { Cooperative detection with GLRT at } \\
1 \text { st tier and OR fusion rule at } 2 \text { nd tier }\end{array}$ & Sbs & Coherent & High \\
\hline [52] & $\begin{array}{l}\text { Rectangular } \\
\text { pulsed based OOK }\end{array}$ & $\begin{array}{l}\text { Nonlinear adaptive threshold detec- } \\
\text { tion based on local geometry and en- } \\
\text { ergy difference of received signal }\end{array}$ & Sbs & Non-coherent & Low \\
\hline [60] & $\begin{array}{l}\text { Rectangular } \\
\text { pulsed based OOK }\end{array}$ & $\begin{array}{l}\text { Two layered detection for reduc- } \\
\text { ing the number of sequences to be } \\
\text { searched }\end{array}$ & Seq & Non-coherent & High \\
\hline [27] & OOK & $\begin{array}{l}\text { ANN with Gradient-Descent opti- } \\
\text { mization for weights }\end{array}$ & Seq & Non-coherent & High \\
\hline [61] & $\mathrm{OOK}$ & Derivative based detection & Sbs & Non-coherent & Moderate \\
\hline [63] & OOK and OSK & $\begin{array}{l}\text { Suboptimal maximum likelihood de- } \\
\text { tector and ISI neglecting detector }\end{array}$ & Sbs & Non-coherent & Moderate \\
\hline [96] & OOK & $\begin{array}{l}\text { ANN with fuzzy threshold detection } \\
\text { and polynomial approximation }\end{array}$ & Sbs & Non-coherent & High \\
\hline [29] & OOK & ANN detection with LM optimizer & Sbs & Non-coherent & Moderate \\
\hline [71] & $\begin{array}{l}\text { Rectangular pulse } \\
\text { based OOK }\end{array}$ & $\begin{array}{l}\text { Binary detection with iterative } \\
\text { method of ISI mitigation }\end{array}$ & Sbs & Non-coherent & Moderate \\
\hline
\end{tabular}

average detection i.e., an average of the output was calculated then fed to the MAP detector for reducing the complexity of the receiver. In the third technique, first-order arrival time was used as the test statistic.

In [104], the transmitter emitted molecules using the OOK modulation scheme. In this work, the channel was similar to the vein and at the receiver, maximum likelihood sequence detection was used. The BER performance was evaluated in the presence of a periodic force generated due to heart pumping, where periodic force was modeled as a sinusoidal wave. Also, the performance evaluation was done considering different sinusoidal phases and the different size of transmitted molecules. Simulation results demonstrated that performance improvement can be achieved by the appropriate selection of release time of molecules and their size.
Synchronous and asynchronous sensing techniques were proposed in [114]. In this work, two different nanonetworks (each consisting of a single transmitter and receiver pair) were assumed inside a blood vessel. At the receiver, energy detection was employed for detecting the molecules. Besides, sensing based on long and short sensing windows in the case of synchronous sensing was also proposed. Moreover, the Bayesian approach was used to derive the likelihood ratio test in the case when the two networks were not synchronized. In [115], sampling time i.e., the number of samples taken at the receiver was optimized to minimize the error probability with OOK modulated transmission. For decoding, an energy detector was used at the receiver. 
Table 3 - Summary of transmission and detection in static MC with drift

\begin{tabular}{|c|c|c|c|c|c|}
\hline Reference & Modulation & Detection & $\begin{array}{l}\text { Symbol- } \\
\text { by-symbol } \\
\text { (Sbs)/Sequence } \\
\text { (Seq) detector } \\
\end{array}$ & $\begin{array}{l}\text { Coherent/ Non- } \\
\text { coherent detection }\end{array}$ & Complexity \\
\hline [98] & $\begin{array}{l}\text { Binary and } \text { M-ary } \\
\text { quantity based } \\
\text { modulation }\end{array}$ & MAP detection & Sbs & Coherent & High \\
\hline [99] & Binary PAM & Weighted sum detector & Sbs & Coherent & Low \\
\hline [100] & OOK & $\begin{array}{l}\text { Detection based on variance } \\
\text { of arrival times of molecules }\end{array}$ & Sbs & Coherent & Moderate \\
\hline [101] & MoSK & CTAT & Sbs & Coherent & Low \\
\hline [102] & OOK & $\begin{array}{l}\text { i) Optimal distributed detec- } \\
\text { tion using weighted Log LRT } \\
\text { ii) } K \text { out of } N \text { fusion rule at FC }\end{array}$ & Sbs & Coherent & $\begin{array}{l}\text { i) High } \\
\text { ii) Low }\end{array}$ \\
\hline [103] & $\begin{array}{l}\text { Binary timing } \\
\text { based modulation }\end{array}$ & $\begin{array}{l}\text { i) MAP detection } \\
\text { ii) Average detection } \\
\text { iii) Order statistic detection }\end{array}$ & Sbs & Coherent & $\begin{array}{l}\text { i) High } \\
\text { ii) Low } \\
\text { iii) Moderate }\end{array}$ \\
\hline [104] & OOK & $\begin{array}{l}\text { Maximum likelihood se- } \\
\text { quence detection }\end{array}$ & Seq & Coherent & Very high \\
\hline [105] & OOK & MF detector & Sbs & Coherent & Low \\
\hline [106] & OOK & SBRNN with ADAM optimizer & Seq & Non-coherent & High \\
\hline [107] & MSSK and QMSSK & $\begin{array}{l}\text { i) Maximum count decoding } \\
\text { ii) Maximum likelihood se- } \\
\text { quence detection } \\
\text { iii) Maximum likelihood de- } \\
\text { tection }\end{array}$ & $\begin{array}{l}\text { Seq for i), ii) and } \\
\text { Sbs for iii) }\end{array}$ & $\begin{array}{l}\text { Non-coherent in i) } \\
\text { and Coherent in ii), } \\
\text { iii) }\end{array}$ & $\begin{array}{l}\text { i) Low } \\
\text { ii) High } \\
\text { iii) Moderate }\end{array}$ \\
\hline [108] & $\begin{array}{l}\text { Release time shift } \\
\text { keying with con- } \\
\text { volutional coding }\end{array}$ & $\begin{array}{l}\text { Viterbi detection with asym- } \\
\text { metric metric }\end{array}$ & Seq & Coherent & High \\
\hline [30] & OOK & Parzen-PNN based detection & Sbs & Non-coherent & High \\
\hline [109] & OOK & $\begin{array}{l}\text { Detection based on i) MAP } \\
\text { criterion ii) Mean square er- } \\
\text { ror iii) Error probability min- } \\
\text { imization }\end{array}$ & Sbs & Non-coherent & $\begin{array}{l}\text { i) Moderate } \\
\text { ii) Low } \\
\text { iii) High }\end{array}$ \\
\hline [110] & $\begin{array}{l}\text { Rectangular pulse } \\
\text { based OOK }\end{array}$ & $\begin{array}{l}\text { Sparse dictionary learning } \\
\text { and Kernel LMS algorithm }\end{array}$ & Sbs & Non-coherent & High \\
\hline [111] & CSK & Fuzzy C-means clustering & Sbs & Non-coherent & Moderate \\
\hline
\end{tabular}

The channel modeling for active and passive receivers was proposed in [116], where reception probability for a passive receiver and the PDF of first hitting time at an absorbing point located on an infinite plane were derived. In addition to this, the reception probability of a molecule was also derived for a receptor on an absorbing wall. Also, maximum likelihood sequence detection using hard decision Viterbi decoding was used at the receiver. In [122], maximum likelihood detection and the detection based of first arrival time of molecules were considered for release time modulation at the transmitter. Authors showed that for the scenarios when the number of released molecules is small, the detector based on first arrival performed very close to the maximum likelihood detector. On the other hand, if the number of released molecules are large, the maximum likelihood detector outperformed the first arrival-time-based detector.

The maximum likelihood sequence detection has been proposed for the M-ary transmission scheme in [123]. In this work, it is shown that the performance can be enhanced by using a shift register of $\log _{2} \mathrm{M}$ bits at the transmitter. The state of shift register determined the number of molecules to be emitted by the transmitter. A sliding bidirectional recurrent neural network has been proposed in [106] for sequence detection. This procedure can be useful when the channel model is unknown. In this work, a sliding window is used over which the estimated PMFs are averaged to find the final PMF.

Super-Paramagnetic Iron Oxide Nanoparticles (SPIONs) were used as the information particles in [124]. In this work, OOK modulation and simple threshold-based detection were used at the transmitter and receiver, respectively. Interestingly, an external magnetic field was used to guide the particles towards the receiver. Thus, the movement of particles was studied under different magnetic field gradients and the system performance was characterized in terms of error rate under different particle size distributions. It is worth noting that this scheme 
Table 4 - Comparison of different modulation schemes proposed in the literature

\begin{tabular}{|l|l|l|l|l|l|}
\hline Reference & Modulation & Abbreviation & Based on & $\begin{array}{l}\text { ISI miti- } \\
\text { gation }\end{array}$ & $\begin{array}{l}\text { Types of } \\
\text { molecule }\end{array}$ \\
\hline \hline $\begin{array}{l}{[32],[39],[24],[50],[61],} \\
{[77],[117],[118],[119]}\end{array}$ & On-off keying & OOK & Concentration & No & 1 \\
\hline$[73],[74],[75],[120]$ & Concentration shift keying & n-CSK & Concentration & No & 1 \\
\hline$[33],[68],[101]$ & Molecular shift keying & n-MoSK & Molecule type & Medium & $n$ \\
\hline$[35],[36]$ & Pulse amplitude modulation & PAM & Concentration & No & 1 \\
\hline$[55],[56]$ & Pulse position modulation & PPM & Molecule release time & No & 1 \\
\hline$[45]$ & $\begin{array}{l}\text { Molecular transition shift key- } \\
\text { ing }\end{array}$ & n-MTSK & $\begin{array}{l}\text { Concentration and } \\
\text { molecule type }\end{array}$ & Yes & $2 \mathrm{n}$ \\
\hline$[63]$ & Order shift keying & OSK & Order of molecules & Yes & 2 \\
\hline$[107]$ & Molecular space shift keying & MSSK & $\begin{array}{l}\text { Spatial position } \\
\text { molecule type }\end{array}$ & Yes & 1 \\
\hline$[107]$ & $\begin{array}{l}\text { Quadrature molecular space } \\
\text { shift keying }\end{array}$ & QMSSK & Yes & 2 \\
\hline$[121]$ & $\begin{array}{l}\text { Depleted-molecular shift key- } \\
\text { ing }\end{array}$ & D-MoSK & Molecule type & Yes & $\mathrm{n} / \log _{2} \mathrm{n}$ \\
\hline
\end{tabular}

can be useful in a negative drift environment where the molecules move away from the receiver. In [108], release time shift keying combined with convolution coding and Viterbi decoding based on asymmetric distance metric was proposed. The basic premise of employing this new metric was the asymmetry of the molecular channel, unlike the symmetric Additive White Gaussian Noise (AWGN) channel. In this work, encoders and decoders based on biological circuits were also proposed, where liposomes capable of receiving and sending molecules were used.

A release time-based modulation scheme was used in [125] for a microfluidic channel with drift. In this scheme, a molecule generator for synthesizing molecules and an oscillator to control their release time were used at the transmitter. On the other hand, the receiver used an oscillator that was not synchronized to the transmitter and NAND gate which could be designed using messenger Ribonucleic acid (mRNA) cells for generating any logical function. For decoding, the receiver employed a maximum likelihood-based detection scheme. The authors demonstrated that in the case of negligible background noise, the variance of the arrival time of molecules follows a non-central Chi-squared distribution. However, for the case when the background noise was present, the PDF of the sample variance of the arrival time was shown to be a linear weighted function of non-central Chi-squared distributions, where the weighting factors followed a hypergeometric distribution.

In [120], three-dimensional molecular cooperative communication system performance was analyzed under the flow-induced diffusive channel. In this work, binary CSK has been used at the transmitter and the equal gain combining was used at the receiver, which combined the signals received from the transmitter and the intermediate cooperative nano-machine. The LRT-based rule was used to determine the detection threshold at the cooperative nano-machine. Moreover, the detection threshold at the final destination was obtained by minimizing the endto-end error probability using the Gradient-Descent algorithm. In addition to decision thresholds, the optimal number of molecules to be transmitted by the source and the cooperative nano-machine was also determined using the Gradient-Descent algorithm. A method for estimating various system parameters in the presence of drift was proposed in [126]. In this work, maximum likelihood estimation was used where the log-likelihood function was maximized to find the diffusion coefficient, the distance between the transmitter and the receiver, and the drift velocity of the medium. The Cramer-Rao lower bound as well as the mean-squared error in the estimation of different parameters were also determined.

A linear filter-based receiver design was proposed in [127] for the time-varying Poisson channel. In this work, a sampled average of expected observed molecules for bit1 and bit- 0 was found over all permutations of sequences. Then, the ratio of these sampled average values was calculated to evaluate filter coefficients. A worst-case filter design was also proposed therein where all previous bits were either 1 or 0 . Finally, filter design based on linear programming was also discussed for a high transmission rate. A fuzzy clustering algorithm called Fuzzy C-mean (FCM) was proposed in [111] where each bit was detected based on its closeness to the center of a particular cluster. The metrics used in this work for calculating the center of the clusters were similar to the ones considered in [69].

\subsubsection{Relay-assisted-based MC systems}

An MC system based on decode-and-forward relaying was analyzed in [128] considering positive drift velocity. For communication and also to avoid interference at the intermediate relay node, type- $A$ molecules were used by the transmitter in the first phase and type- $B$ molecules were used by a relay node in the second phase. The error proba- 
bility expression at the destination was derived which was shown to be a function of detection thresholds employed at the relay and destination nodes. Therefore, the authors determined: (i) the threshold at the relay node by using the likelihood-ratio test and (ii) the threshold at the destination by minimizing the end-to-end error probability. This end-to-end error probability minimization problem was formulated as a quasi-convex optimization problem, which was solved using the bisection method.

The work in [129] proposed an estimate-and-forward relaying scheme under the flow-induced diffusive channel. In this scheme, estimating the number of transmitted molecules at the relay node was done by maximizing the joint PDF of all observations. The Newton-Raphson method was used for this purpose. Moreover, the analytical expression of error probability was derived considering maximum likelihood and energy detectors at the receiver. Simulation results demonstrated that the decodeand-forward relaying achieves low BER if the relay is located close to the source. On the other hand, estimateand-forward relaying works better if the relay is located close to the destination. The work in [109] considered an amplify-and-forward relaying scheme to increase the range of communication. Three different detection techniques were presented considering 00K-based transmission. In particular, the detection based on mean-squared error, the detection based on MAP, and the decision rule which minimized the error probability were considered.

\subsubsection{MIMO-based MC systems}

In [130], the MISO system has been considered where multiple transmitters were assumed to send information in different time slots using OOK modulation. Apart from ISI, ILI was also considered while analyzing system performance. In this work, symbol duration and the number of transmitted molecules were jointly optimized. For this purpose, multi-objective optimization was transformed to a single objective optimization by using the weighted sum method. This system was claimed to be useful for drug release management where the requirement of various drugs in different dosages was present.

Various index modulation schemes utilizing antenna separation were proposed in [107]. The first scheme considered Molecular Space Shift Keying (MSSK) modulation, where the transmitter uses each of its antennas to transmit different bit-streams. The second scheme considered Quadrature Molecular Space Shift Keying (QMSSK) where two different MSSK modulators were used at the transmitter and each modulator sent a different type of molecule thus providing channels orthogonal to each other. The schematic representation of both MSSK and QMSSK schemes are given in Fig. 16. Also, a combination of MSSK and QMSSK was proposed as the third modulation scheme. Three different detection schemes i.e., maximum-count decoding, maximum likelihood sequence detection, and symbol-by-symbol maximum likelihood detection were studied.
In maximum-count decoding, one receiver antenna index is found that receives the maximum number of molecules. Corresponding to that antenna index, an $n$-bit sequence is decoded where $n=\log _{2}\left(n_{t}\right)$ and $n_{t}$ is the number of transmit antennas. In maximum likelihood sequence detection, the likelihood function corresponding to a particular symbol sequence is maximized. Towards this, the maximum likelihood sequence detector generates the trellis to obtain the likelihood of $n_{r}^{L}$ antenna index vectors, where $n_{r}$ and $L$ represent the number of receiver antennas and channel memory length, respectively. On the other hand, the symbol-by-symbol maximum likelihood detection decodes the symbol by maximizing the sum of likelihood functions at each of the receiver antennas.

\subsubsection{Distributed detection-based MC systems}

A distributed detection scheme was proposed in [131] and [102], where many sensors sent molecules to an FC to decide the presence or absence of a biological agent (BA). In this work, the emission by the BA was modeled as the Kolmogorov-Feller diffusion process (colloidal Brownian motion under drift). The weighted log-likelihood ratio test was the optimal fusion rule for binary hypothesis testing. This rule is called the Chair-Varshney rule under both the NP and Bayesian framework. Further, a suboptimal fusion rule was also presented therein. Event detection in anomalous diffusion with drift was studied in [132] for an FC-based distributed nano-network. Considering OOK modulated transmission, the detection at FC was carried out using the binary hypothesis testing with LLR. In this work, time-slot optimization based on reinforcement learning was also proposed to increase the throughput of the network.

\subsubsection{Machine-learning-based MC systems}

High-dimensional metric combining was proposed in [30] for a non-coherent detection scheme. In this work, the first metric was constructed using the rising edge property of the signal, the second metric was the minimum inflection considering successive time slots and the third metric was the energy difference in successive time slots. At the receiver, the sum of these metrics was used for detection, which was shown to be insensitive to ISI and imperfect synchronization. Parzen-probabilistic Neural Network (PNN) was trained using the high-dimensional metric and corresponding symbols to obtain the approximate likelihood PDFs instead of exact likelihood PDFs for detection. Also, Parzen-PNN was claimed to be less complex than the back-propagation based ANNs and radial basis function-based neural networks.

A non-linear equalizer, which mitigates ISI and counters the non-linearity of the channel has been proposed in [110]. In this work, lower-to-higher dimensional mapping was carried out using a machine learning algo- 

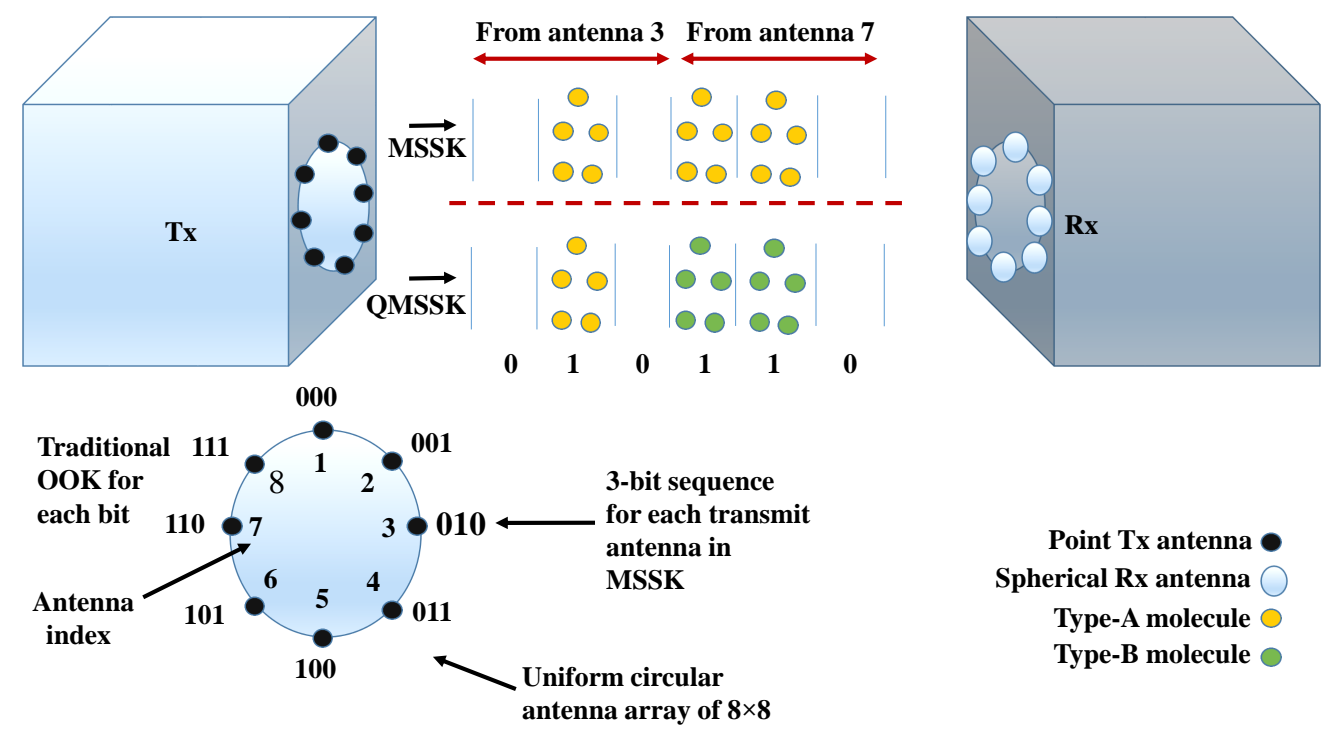

Fig. 16 - MSSK and QMSSK modulation schemes. In $n_{t}$-QMSSK scheme, first $\log _{2} n_{t}$ bits sent using type- $A$ molecules and last log $n_{t}$ bits sent using type- $B$ molecules. $n_{t}=8$ is used.

rithm, Reproducing Kernel Hilbert Space (RKHS). Moreover, sparse dictionary learning and the Kernel LMS algorithm were used by the receiver for detection. Also, the stochastic Gradient-Descent approach was used for updating the weights.

\subsection{Performance and complexity comparison of different detection techniques for static MC}

If $N_{s}$ denotes the total number of samples taken by the receiver in a bit interval then the computational complexity of the linear MMSE method is $O\left(N_{s}^{3}\right)$. The complexity of the coherent MAP method [40], [98] is $O\left(2^{N_{s}}\right)$. The derivative-based detector [61] offers less complexity and better BER than the MAP detector [40]. Further, the noncoherent detector based on concentration difference in [49] has a computational complexity of $O\left(N_{s}^{2}\right)$. Furthermore, the non-linear detector in [69] offers less computational complexity than the coherent MAP and MMSE detectors. With the channel coding scheme used in [58], the complexity of maximum likelihood sequence detection is $O(K \log (\log (S)))$ where $K$ is the codeword length and $S$ is the number of available symbols at the transmitter. To reduce the complexity of decoding the convolutional codes, Viterbi detector with asymmetric distance metric has been proposed in [108]. The complexity of the receiver in [75] is $O(K / 2)$.

Both decision feedback and the blind detectors had a linear complexity in $n$ in [65] but the blind detector is less complex than the decision feedback detector since it does not needs the calculation of the complex decision metric and the statistical CSI. Here $n$ denotes the sequence length to be decoded. The CSI free detector proposed in [69] gives a complexity of $O\left(S N_{s}\right)$. The MSSK mod- ulation helps to mitigate the ILI while the QMSSK mitigates ISI significantly in MIMO systems [107]. Further, a simple maximum count decoding introduced in [107] offers less computational complexity than other existing detection schemes for MIMO systems. Also, the complexity of estimate-and-forward relaying is the highest but it gives improved performance than decode-and-forward, amplify-and-forward relaying. The complexity of decodeand-forward relaying increases with the modulation order [74] while amplify-and-forward relaying offers the least computational complexity.

Further, the non-linear receiver based on sparse dictionary learning and the Kernel LMS algorithm [110] gives a complexity of $O\left(\left|D_{m}\right|\right)$ where $\left|D_{m}\right|$ is the number of observations present in the dictionary at convergence. Furthermore, a low complexity detection $\approx O\left(N_{s}^{2}\right)$ was proposed in [72]. In [111], the non-coherent detection based on Fuzzy-C means clustering gives a computational complexity of $O\left(S N_{k}\right)$ that is significantly less than the coherent MAP detection. Here $N_{k}$ is the number of data points in a cluster. Time complexity of the ANN based detector proposed in [27] was shown as $O\left(\sum_{i=1}^{d} n_{i-1} s_{i}^{2} f_{i} m_{i}^{2}\right)$ where $i$ is the index of a layer, $d$ is the number of layers, $n_{i-1}$ is the number of input channels of the $i$ th layer, $s_{i}$ is the spatial size of filter, $f_{i}$ is the number of filters in the $i$ th layer, and $m_{i}$ is the spatial size of the output feature. Further, in the Parzen-PNN technique proposed by [30], the complexities related to computation, time and storage are $O\left(d N_{s}\right), O(d)$, and $O\left(d N_{s}\right)$, respectively. Here, $d$ denotes the dimension of the metrics and $d=3$ was used in [30]. The Parzen-PNN-based detector is less complex than the ANN-based detectors. Table 3 summarizes the modulation and detection techniques in static MC with drift in the channel. 
Further, Table 4 compares the various modulation schemes in terms of their ISI mitigation capability and the types of molecules required. In traditional 00K, no ISI mitigation is possible while the MoSK modulation suppresses the ISI moderately. The types of the molecule required in MoSK increase with the modulation order n. The MTSK [45] modulation also mitigates the ISI but the types of the molecule required are larger than MoSK. Further, OSK [63] has a good ISI mitigation capability and the types of molecules required are only 2. D-MoSK [121] is a modified version of MoSK modulation that needs fewer types of the molecule than MoSK for a given modulation order. Also, MSSK and QMSSK [107] modulations use antenna separation to suppress the ISI and the ILI, and the types of the molecule required are less than most of the modulation schemes.

\subsection{Challenges in detection and possible solutions}

\subsubsection{Noise and ISI in diffusive MC channel}

The major challenge in detection arises due to noise and ISI experienced in the diffusing MC channel even for the static scenario where the distance between transmitter and receiver is constant. The number of received molecules $N_{\mathrm{rx}}(r, t)$ at time $t$ and at a distance $r$ from a point transmitter is given by [133]

$$
N_{\mathrm{rx}}(r, t)=\frac{N_{\mathrm{tx}} V_{\mathrm{rx}}}{(4 \pi D t)^{3 / 2}} e^{-r^{2} / 4 D t},
$$

where $N_{\mathrm{tx}}$ represents the number of transmitted molecules, $V_{\mathrm{rx}}$ is the volume of the receiver, $D$ is the diffusion coefficient of a signaling molecule. Since the distance $r$ between the communicating nano-machines is constant, the time at which $N_{\mathrm{rx}}(r, t)$ is maximum, can be obtained by differentiating (6) with respect to $t$ and set it equal to zero, as

$$
t_{\text {peak }}=\frac{r^{2}}{6 D}
$$

Also, the peak amplitude can be obtained by substituting (7) in (6)

$$
\left[N_{\mathrm{rx}}(r, t)\right]_{\max }=\left(\frac{3}{2 \pi e}\right)^{3 / 2} \frac{N_{\mathrm{tx}}}{r^{3}} .
$$

It can be observed from (8) that the peak amplitude is independent of the diffusion coefficient $D$; however, varies inversely as the third power of distance $r$. For the case when $r$ and $D$ are constant, and the noise and ISI are absent, as shown in Fig. 13, then detection at the receiver can be easily performed by sampling at a fixed peak time $t_{\text {peak }}$ and comparing $N_{\text {rx }}\left(r, t_{\text {peak }}\right)$ against a threshold.

The threshold can be obtained by applying MAP-based rule [80] to the PDF of the received signals, as shown in Fig. 17. According to the MAP-based rule, the optimal threshold required for detection in $j$ th bit-interval for
Gaussian distributed signals is given by

$$
\lambda_{j}=0.5 \frac{\mu_{j 1}+\mu_{j 0}}{\mu_{j 1}-\mu_{j 0}} \sigma^{2} \ln \left(\frac{\mathrm{P}\left(b_{j}=1\right)}{\mathrm{P}\left(b_{j}=0\right)}\right),
$$

where $\sigma^{2}$ is the AWGN variance. Further, for Poisson distributed signals, the detection threshold in $j$ th bit-interval [133] can also be obtained by substituting PDF/PMF equations in LRT expression (shown in Fig. 17) as

$$
\lambda_{j}=\frac{\ln \left(\frac{\mathrm{P}\left(b_{j}=1\right)}{\mathrm{P}\left(b_{j}=0\right)}\right)+\mu_{j 1}-\mu_{j 0}}{\ln \left(\mu_{j 1}\right)-\ln \left(\mu_{j 0}\right)} .
$$

In (9) and (10), the quantities $\mu_{j 1}$ and $\mu_{j 0}$ denote the mean of received signals corresponding to the transmission of bit- 1 and bit- 0 , respectively. The probabilities $\mathrm{P}\left(b_{j}=1\right)$ and $\mathrm{P}\left(b_{j}=0\right)$ represent the a priori probabilities for bit1 and bit-0, respectively. For the case when the noise and ISI are present, as shown in Fig. 14, sampling at $t_{\text {peak }}$ can cause incorrect detection. The received signal including ISI (for OOK at transmitter) is given as

$$
y(t)=\sum_{j=0}^{\infty} b_{j} N_{r x}\left(r, t-j T_{b}\right)+n(r, t),
$$

where $b_{j} \in\{0,1\}$ is transmitted bit in $j$ th bit-interval, $T_{b}$ is the bit duration and $n(r, t)$ is the signal dependent noise whose variance is given as [39]

$$
\sigma^{2}[n(r, t)]=\frac{3}{4 \pi r_{\mathrm{rx}}^{3}} N_{\mathrm{rx}}(r, t),
$$

where $r_{\mathrm{rx}}$ is the radius of the receiver.

Fig. 14 shows the received signal perturbed by the noise and ISI for the transmitted bit sequence $\left[\begin{array}{lllll}1 & 1 & 0 & 1 & 0\end{array}\right]$. In the $3 \mathrm{rd}$ and 5 th bit-intervals the transmitted bit was 0 , but the maximum signal in those bit-intervals is above the threshold that lead to incorrect decoding if we employ an asynchronous peak detector. In the MC channel, the noise can be filtered out, as shown in [34] and maximum likelihood sequence-based detection can be used at the receiver to enhance the system performance under ISI. Also, MMSE equalizer and DFE can be considered for ISI mitigation $[40,45]$. It is worth noting that these equalizers are less complex than the maximum likelihood sequencebased detection. Moreover, the detection in the presence of time varying diffusion coefficient ${ }^{8}$ can also be done by using a channel estimator proposed in [40].

\subsubsection{Unknown channel model}

If the channel model is not known at the receiver then non-coherent detection schemes such as [52] can be employed, which relies on the local geometry of the received signal and the energy difference between received signals

\footnotetext{
${ }^{8} \mathrm{~A}$ time-varying diffusion coefficient can exist in multilayered channels such as alveolar-blood barrier [134].
} 


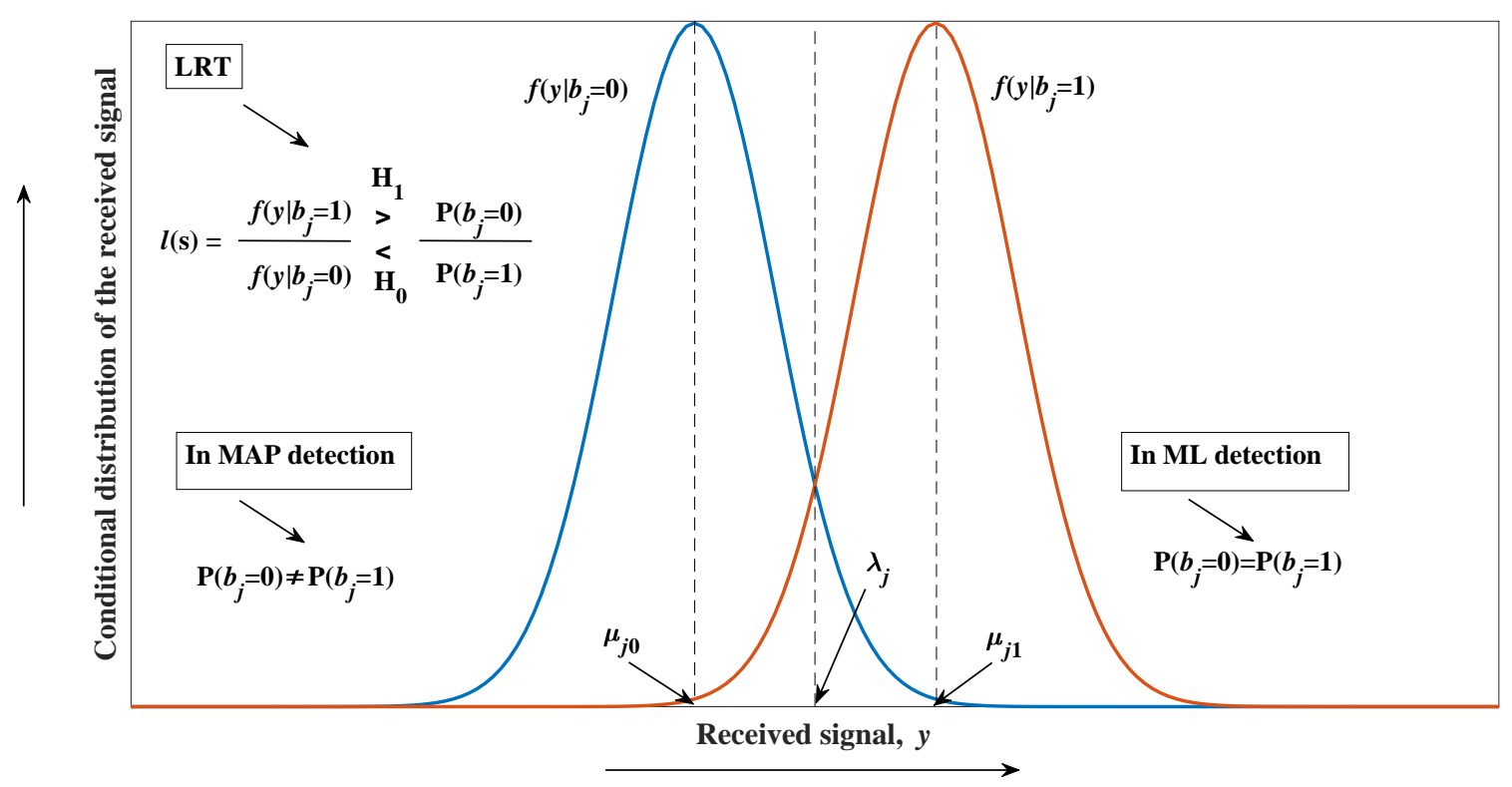

Fig. 17 - Conditional distribution of the received signal.

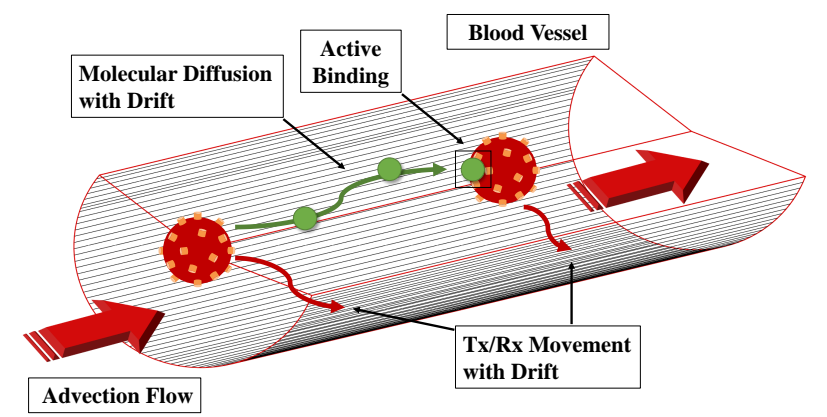

Fig. 18 - Mobile nano-machines under drift and diffusion inside the blood vessel.

in consecutive bit intervals. Further, ANN-based detectors can also be very robust [106] under unknown channel conditions. These detectors can be trained on the transmitted bit sequence and the corresponding received signal or features extracted from the received signal. Also, unsupervised clustering [111] based on a fuzzy clustering algorithm can be useful in unknown channel conditions.

\section{TRANSMISSION AND DETECTION WITH MOBILE NANO-MACHINES}

This section presents various transmission and detection schemes for mobile nodes. Performance and complexity comparison of the detection schemes are also discussed. An illustration of mobile nano-machines inside the blood vessel is shown in Fig. 18.

\subsection{Mobile nano-machines in pure diffusive channel}

\subsubsection{Single transmitter and receiver-based mo- bile MC systems}

A single sample detector at a fixed sampling time in each bit-interval was proposed in [117] for diffusion-based MMC, where the OOK modulation scheme was considered at the transmitter. In this work, dynamic CIR was modeled by using the effective diffusion coefficient value ${ }^{9}$ in the expression of static CIR. No noise and ISI were considered while analyzing the system performance in terms of error rate. The error probability of the order of $10^{-4}$ was obtained in the case of the low diffusion coefficient of the transmitter (i.e., $10^{-12} \mathrm{~m}^{2} / \mathrm{s}$ ). However, an increase in the diffusion coefficient by 100 times led to degradation in BER performance by approximately 100 times indicating that the fixed sampling time does not work well for MMC.

A non-coherent detection scheme based on the local convexity of the received signal has been proposed in [135]. In this work, the received signal was filtered using a moving average filter. Three convexity metrics have been found and added together to yield the final decision metric. This decision metric was compared with a threshold to decide in favor of bit- 1 or bit- 0 . Note that the convexity metric was higher in the case of bit-1. Also, the upper and lower bounds on the detection threshold were evaluated. Finally, the authors showed that the complexity of the convexity-based detector was lower than the MAP and MMSE detection schemes, however, the proposed detection scheme achieved the identical BER values as the MAP and MMSE detectors at a higher SNR regime.

\footnotetext{
${ }^{9}$ Effective diffusion coefficient is calculated as the sum of diffusion coef-
} ficients of signaling molecules and the receptors. 
Two methods of adaptive threshold detection for 0OK transmission were proposed in [118]. The adaptive threshold calculation was based on determining the average distance variation within a symbol interval. The distance was calculated using the diffusion equation (6). The first adaptive detection scheme was based on a comparison of received concentration with the adaptive threshold. In this scheme, the adaptive threshold was selected as half of the maximum value of the reconstructed signal during the interval where the last bit- 1 was detected. Thus, the decision rule to detect the transmitted bit in the $j$ th bit interval can be expressed as

$$
\hat{b}_{j}= \begin{cases}1 & \text { if } h_{j, \text { peak }} \geq h_{*, \text { peak }} / 2 \\ 0 & \text { otherwise }\end{cases}
$$

where $*$ denotes the index of the last bit- 1 detected, and $h_{j, p e a k}$ is the peak value of reconstructed signal using average distance between the transmitter and the receiver in $j$ th bit interval.

The second detection scheme was peak time-based detection in which the peak time of the current bit-interval was compared with 1.6 times the peak time of the reconstructed signal where the last bit-1 was detected. Moreover, ISI mitigation was also performed by subtracting the reconstructed signals of previous bit-intervals from the total received signal in the current bit-interval. Simulation results demonstrated that these detection schemes perform well if the coherence time of the channel is large, however they fail to give satisfactory performance if the coherence time is small (i.e., around 1-bit interval).

To overcome the limitations of this work, another detection scheme was proposed in [136] in which modified concentration shift keying was used where some molecules for bit- 0 were also transmitted. This could help the receiver to find the distance using the diffusion equation even when bit- 0 was transmitted and the adaptive threshold was calculated based on the reconstructed signals of previous bit duration unlike [118] where the threshold calculation based on the bit duration in which the last bit-1 was transmitted. The detection rule in [136] is given by

$$
\hat{b}_{j}= \begin{cases}1 & \text { if } \max \left(x_{r_{k}, k}[j]\right) \geq \frac{f_{1, \max }(j-1, t)-f_{0, \max }(j-1, t)}{\ln \frac{f_{1, \max }(j-1, t)}{f_{0, \max }(j-1, t)}} \\ 0 & \text { otherwise. }\end{cases}
$$

where $x_{r_{k}, k}[j]$ is the ISI mitigated signal in $j$ th bit interval, $r_{k}$ is the distance between the transmitter and the receiver at $k$ th sample, $f_{1, \max }(j-1, t)$ and $f_{0, \max }(j-1, t)$ are the peak values of the reconstructed signals for bit- 1 and bit- 0 respectively in $(j-1)$ th bit interval. It is shown that the detection scheme in [136] performs well for a coherence time of $\approx 2$-bit duration.
Further, a signal detection scheme with initial distance estimation was proposed in [137]. In this scheme, a pilot signal was released before information transmission which assisted to estimate the stochastic distance based on maximizing the likelihood of the received signal in the first step. In the second step, maximum likelihood estimation was used to find the initial distance by maximizing the likelihood function based on the PDF of distance. Authors employed a MAP-based detection scheme at the receiver and based on this, the expression for optimal threshold was obtained. Furthermore, an adaptive detection scheme for OOK modulation was proposed in [138], where the decision threshold in the current bit-interval was selected as the weighted sum of the received signals in the previous two bit-intervals. The weights were obtained by minimizing the error rate at the receiver nanomachine. Also, a pre-coding scheme was presented to combat ISI, where the transmitter scaled the number of released molecules if two consecutive bit-1 were transmitted. The probability of error was further minimized to determine the optimal scaling factor. The proposed technique outperformed the fixed threshold technique and the one presented in [47] where the received signal in the previous bit-interval was considered as an adaptive threshold.

Optimal LLR based decision rule was derived at the receiver in [139]. Based on the derived optimal rule, the probability of error with respect to the number of transmitted molecules and channel capacity with increasing external interference was also evaluated. A detection scheme based on slope values within a symbol interval was proposed in [140] considering 00K based transmission at the transmitter. In this work, Einsteins' law of diffusion was used to find the CIR. Moreover, the detection threshold was set to zero as the slope value was positive for bit- 1 and negative for bit-0. Cell-to-cell communication based on calcium signaling was proposed in [141]. In this work, intra-cellular and intercellular calcium signaling was studied for mobile cells in an environment composed of cytoplasm. Based on the external diffusion model of calcium, the transmission of calcium-based on concentration and waveform was also studied. Moreover, the decay of calcium signals in the environment and the number of activated cells as a percentage of sender cells were analyzed.

The work in [142] studied cluster formation by a set of mobile nano-machines using the attractant molecules. This work considered that the angular movement of nanomachines was in the direction of the maximum concentration gradient and the concentration sensed by nanomachines was governed by their sensitivity. The number and size of clusters were shown to be controlled by the sensitivity of nano-machines to the concentration of attractants. An MMC system for drug delivery was proposed in [143] where drug carriers and diseased cells were considered as transmitters and receivers, respectively. Using the PDF of stochastic CIR and the reception probability 
of a single molecule, the authors demonstrated that the controlled drug release mechanism was shown to be better than the constant drug release. Moreover, the PDF of the dynamic distance between the transmitter and the receiver was used to optimize the release profile at the transmitter, the time duration of a frame, and the detection threshold at the receiver.

A non-coherent iterative detection has been proposed in [144] considering the 1-D motion of transmitter and receiver, where the PDF of first hitting time was obtained in terms of effective diffusion coefficient [117]. In this work, ISI mitigation was also implemented by subtracting the average ISI concentration from the total received signal in the current symbol-interval. Another non-coherent detection scheme for 00K was proposed in [145], where the decision metric was calculated as the difference of the signal energies received in current and previous bitintervals. This technique is useful for the scenarios that experience strong ISI because it makes the energy difference positive in the case of bit-1 and negative in the case of bit- 0 .

In [133], the statistical nature of CIR in the case of MMC was investigated, where the mean and variance of the noiseless and noisy received signal were derived. The noisy received signal was shown to be Poisson-Log normal distributed which could be approximated as Poisson distributed in some cases. In this work, the PDF of the dynamic distance was also found. Moreover, binary hypothesis testing was done at the receiver for three different detection thresholds i.e. fixed threshold, half threshold, and optimal threshold according to MAP rule for Poisson distributed signals. Simulated results demonstrated that the MAP-based optimal thresholding scheme achieved the lowest BER and on the other hand, the half threshold scheme performed the worst.

In [146], two transmission techniques based on CSK and Manchester coding were presented, where bit- 1 and bit- 0 were represented as symbol [ 10 l 0 ] and [0 1], respectively. Moreover, at the receiver, a concentration differencebased detection was proposed which used the maximum concentration difference within a bit-interval for detection of the transmitted bit using CSK. For Manchester coding at the transmitter, the detector employed a decision metric based on concentration difference between successive bits and the detection threshold was found using the MAP rule.

In [119], OOK modulation has been used at the transmitter and an adaptive detection scheme based on local convexity of the received signal in the case of bit- 1 and concavity of the received signal in the case of bit- 0 was proposed for the receiver. In this work, the average of the received signal was found where the average was calculated for the two sampling times within which the signal was convex. For example, $0.1 \mathrm{~s}$ and $0.3 \mathrm{~s}$ in Fig. 13. This average value was later subtracted from the peak received signal within a bit-interval for designing a convexity metric. Moreover, an adaptive threshold was obtained as the weighted sum of convexity metrics of the bit-intervals in which the last, the second last, and the third last bit-1 were detected.

\subsubsection{Relay-assisted-based mobile MC systems}

Forster Resonance Energy Transfer (FRET ${ }^{10}$ )-based MMC has been proposed in [147] for detecting tumors. In this work, authors considered two different types of network configuration: (i) FRET-based mobile sensor and actuator network in which molecular sensing and actuating tasks were carried out at the molecular level, (ii) FRET-based mobile ad-hoc network with source, relay, and destination nodes, where the source transmits information to the relay node or destination in a probabilistic manner. Detection probability and detection time of a single message were analyzed, where message propagation is modeled using the Markov-chain process.

\subsubsection{MIMO-based mobile MC systems}

In [148], MIMO-MC was proposed for both static and dynamic transmitter and receiver scenarios. Before sending a block of data, a training sequence was sent for channel estimation. Channel estimation was done using the maximum likelihood CIR and the least square CIR estimators, where the latter was less complex to implement. In this work, the training sequence was selected which maximized the Cramer-Rao bound of the CIR. The authors also studied the performance of DFE, MMSE-DFE, and ZF-DFE equalizers for ISI/ILI mitigation. Moreover, to avoid cross-talk, time interleaving was used, where different gates at the transmitter released molecules at different time intervals within a bit duration. For performance evaluation, the authors showed that MSEs for the least-squares CIR estimator and maximum likelihood CIR estimator were $16 \mathrm{~dB}$ and $14 \mathrm{~dB}$, respectively, at a training sequence of length 250 bits. Further, with a decrease in training sequence length from 250 bits to 16 bits, the MSEs for least squares and maximum likelihood CIR estimators increased to 25 and $23 \mathrm{~dB}$, respectively.

\subsubsection{Machine-learning-based mobile MC systems}

Various symbol-by-symbol and sequence detectors based on neural network ${ }^{11}$ for binary and M-ary amplitude modulation schemes were proposed in [31]. Specifically, Recurrent Neural Network (RNN), Bidirectional RNN (BRNN), and Sliding BRNN (SBRNN) with LSTM cells were used, where SBRNN was shown to perform well for a coherence time of $\approx 1$-bit duration.

\footnotetext{
${ }^{10}$ FRET is an energy transfer mechanism observed in fluorophores.

${ }^{11} \mathrm{~A}$ significant advantage of neural network-based detectors is that they can perform well in time-varying channels where CSI is difficult to obtain at the receiving node.
} 
Besides, a comparison with Viterbi sequence detection was also presented in [31].

Further, the work in [149] proposed an ANN-based detection scheme using Broyden-Fletcher-Goldfarb-Shanno (BFGS) algorithm where received signal was filtered then the filtered signal, slope values and the concentration difference values of the filtered signal were used for training and detection by the NN. Filtering the received signal was done by partitioning the bit interval into $P$ equal subintervals and averaging the signal in each partition as described below.

$$
\phi_{i}=\frac{1}{N_{p}} \sum_{k=j N_{s}+l o w}^{j N_{s}+h i g h} y_{j}[k],
$$

where $i=0,1,2, \cdots, P-1$ and $N_{p}$ is the number of samples within a partition. The low and high parameters are defined as, low $=i N_{p}$ and high $=(i+1) N_{p}-1$ for $\phi_{i}$. Using (15), the filtered signal in $j$ th bit interval can be written as $\boldsymbol{\phi}_{\boldsymbol{j}}=\left[\phi_{0}, \phi_{1}, \cdots, \phi_{P-1}\right]$. Based on this filtered signal, the slope vector $s_{j}$ and concentration difference [146] vector $\boldsymbol{d}_{\boldsymbol{j}}$ can be obtained as $\boldsymbol{s}_{\boldsymbol{j}}=\left[s_{0}, s_{1}, \cdots, s_{P-2}\right]$, where $s_{i}=\phi_{i+1}-\phi_{i}$ and $\boldsymbol{d}_{\boldsymbol{j}}=\left[d_{0}, d_{1}, \cdots, d_{P-2}\right]$, where $d_{i}=\phi_{i+1}-\phi_{0}$. Thus, the features $z_{j}$ (input to the detector) for the three different techniques were based on (a) the filtered signal, (b) slope, and (c) concentration difference:

$$
z_{\boldsymbol{j}}= \begin{cases}\boldsymbol{\phi}_{\boldsymbol{j}} & \text { for (a), } \\ {\left[s_{0}, s_{1}, \cdots, s_{P-2}, E\left(\boldsymbol{s}_{\boldsymbol{j}}\right), \operatorname{var}\left(\boldsymbol{s}_{\boldsymbol{j}}\right)\right]} & \text { for (b), } \\ {\left[d_{0}, d_{1}, \cdots, d_{P-2}, E\left(\boldsymbol{d}_{\boldsymbol{j}}\right), \operatorname{var}\left(\boldsymbol{d}_{\boldsymbol{j}}\right)\right]} & \text { for (c), }\end{cases}
$$

where $E(\cdot)$ and $\operatorname{var}(\cdot)$ denote the expectation and the variance. In this work, the transmitted bits are represented in the following manner. For bit- $0, \boldsymbol{p}_{\boldsymbol{j}}=\left[\begin{array}{ll}0 & 1\end{array}\right]^{T}$ and for bit- $1, \boldsymbol{p}_{\boldsymbol{j}}=\left[\begin{array}{ll}1 & 0\end{array}\right]^{T}$. Note that the values inside $\boldsymbol{p}_{\boldsymbol{j}}$ represent the Probability Mass Function (PMF) of the transmitted bit. If $L$ consecutive bits are transmitted then $P_{L}=$ $\left[\boldsymbol{p}_{1}, \boldsymbol{p}_{2}, \cdots, \boldsymbol{p}_{\boldsymbol{L}}\right]$ represents the sequence of transmitted bits and $Z_{L}=\left[z_{1}, z_{2}, \cdots, z_{L}\right]$ be the corresponding features then the training data set is represented by $\left(P_{L}, Z_{L}\right)$. The output of the NN is the vector $\hat{\boldsymbol{p}}_{\boldsymbol{j}}=N N\left(\boldsymbol{z}_{\boldsymbol{j}} ; \boldsymbol{W}\right)$, where $W$ represents the weights and biases in the NN. Finally, the bits are estimated according to the following criterion

$$
\hat{b}_{j}=\arg \max _{b_{j} \epsilon S} \hat{\boldsymbol{p}}_{\boldsymbol{j}},
$$

where $\hat{\boldsymbol{p}}_{\boldsymbol{j}}=\left[\operatorname{Pr}\left(b_{j}=1 \mid z_{\boldsymbol{j}}\right) \operatorname{Pr}\left(b_{j}=0 \mid z_{\boldsymbol{j}}\right)\right]^{T}$ is the estimated PMF vector.

Furthermore, a detection scheme based on GenerativeAdversarial-Network (GAN) has been proposed in [150]. This network learned from the channel transition probability. For fine tuning the transition probabilities, the scheme in [150] combined the use of pilot symbols and generating training data in real time to track time-varying channels. Most importantly, this scheme avoids retraining the network from the beginning.

\subsection{Mobile nano-machines in flow-induced diffusive channel}

\subsubsection{Single transmitter and single receiver- based mobile MC systems}

A quaternary modulation scheme for reducing ISI was proposed in [121], where type- $A$ and type- $B$ molecules were used for transmission. In this scheme, no molecules were sent for symbol [0 0], type-A molecules were sent for [0 1], type-B molecules were sent for [1 0], and both types molecules were sent for symbol [1 1]. This scheme was termed as Depleted-MoSK (D-MoSK). For decoding purposes, maximum likelihood detection was used at the receiver. Individual thresholds for both types of molecules were used at the receiver e.g. if the number of type-A molecules was above a certain threshold $t_{a}$ and the number of type-B molecules was less than the threshold $t_{b}$ then the transmitted symbol was decoded as [ $\left[\begin{array}{ll}0 & 1\end{array}\right]$.

On similar lines, other symbols were decoded. Simulation results showed that the D-MoSK modulation scheme resulted in a better BER performance than MoSK. Further, a novel modulation based on permutation of different molecules was proposed in [153] to achieve better performance than D-MoSK in the presence of strong ISI. In this modulation scheme, different types of molecules were sent at different instants within a symbol duration. If $M$ different types of molecules are chosen then $M$ ! permutations exist and in this case a total $\left[\log _{2} M\right.$ ! $\rfloor$ bits per symbol can be sent. At the receiver, maximum likelihood detection was performed. This modulation scheme achieved better BER performance than MoSK and D-MoSK schemes for a large ratio of number of transmitted molecules to information bits per symbol.

In [154], a nano-sensor network for in-body applications has been proposed in which various nano-machines used repellent molecules to move away from one another. This process is suitable for searching a target inside the body. Further, attractant molecules were used by the nanomachines to come closer once the target is detected. In this work, a non-diffusion ${ }^{12}$-based MC has been assumed and the gradient of adhesive molecules which could bind to the inner wall of the blood vessel were used for guiding the mobile nano-machines. This kind of coordination can be useful where loss of molecules due to diffusion is high. More specifically, the aim of this work was to evaluate the number of nano-machines near to the target and the number of nano-machines hitting the target.

Furthermore, a leader follower-based MMC has been discussed in [155]. After finding the target location, the leader nano-machines released adhesive molecules which could be sensed by the follower nano-machines for moving towards a target (e.g., cancer cells) for various purposes such as drug delivery etc. Maximum likelihood esti-

\footnotetext{
${ }^{12}$ In non-diffusion-based MC, molecules disperse quickly in the environment, which has dominant flow and limited diffusion.
} 
Table 5 - Summary of transmission and detection in MMC with and without drift

\begin{tabular}{|c|c|c|c|c|c|}
\hline Reference & Modulation & Detection & $\begin{array}{l}\text { Symbol- } \\
\text { by-symbol } \\
\text { (Sbs)/Sequence } \\
\text { (Seq) detector }\end{array}$ & $\begin{array}{l}\text { Coherent/ } \\
\text { Non-coherent } \\
\text { detection }\end{array}$ & Complexity \\
\hline [117] & OOK & Single sample detection & Sbs & Non-coherent & Low \\
\hline [135] & OOK & Local convexity detection & Sbs & Non-coherent & Moderate \\
\hline [118] & OOK & $\begin{array}{l}\text { Adaptive threshold-based detection } \\
\text { with distance estimation in intervals } \\
\text { with bit-1 (Valid for large coherence } \\
\text { time) }\end{array}$ & Sbs & Coherent & Very high \\
\hline [136] & MCSK & $\begin{array}{l}\text { Adaptive threshold detection with } \\
\text { distance estimation in every bit- } \\
\text { interval (Valid for small coherence } \\
\text { time) }\end{array}$ & Sbs & Coherent & Very high \\
\hline [31] & $\begin{array}{l}\text { Binary and M- } \\
\text { ary amplitude } \\
\text { modulation }\end{array}$ & $\begin{array}{l}\text { Recurrent neural network detection } \\
\text { schemes }\end{array}$ & Sbs and Seq & Non-coherent & High \\
\hline [144] & OOK & Iterative detector & Sbs & Non-coherent & High \\
\hline [139] & OOK & LLR test & Sbs & Coherent & Moderate \\
\hline [148] & $\begin{array}{l}\text { OOK } \\
\text { MC) }\end{array}$ & $\begin{array}{l}\text { i) DFE, ii) MMSE-DFE, iii) ZF-DFE, and } \\
\text { iv) Least squares DFE detector }\end{array}$ & Sbs & Coherent & $\begin{array}{l}\text { i) Very high } \\
\text { ii) High } \\
\text { iii) High } \\
\text { iv) Very high }\end{array}$ \\
\hline [138] & OOK & Adaptive threshold detector & Sbs & Non-coherent & Moderate \\
\hline [145] & OOK & Energy difference based detector & Sbs & Non-coherent & Low \\
\hline [133] & CSK & $\begin{array}{l}\text { Binary detector with i) Fixed thresh- } \\
\text { old, ii) Half threshold, iii) Optimal } \\
\text { threshold obtained using MAP rule }\end{array}$ & Sbs & Non-coherent & $\begin{array}{l}\text { i) Low } \\
\text { ii) Low } \\
\text { iii) High }\end{array}$ \\
\hline [146] & $\begin{array}{lr}\text { MCSK, } & \text { Manch- } \\
\text { ester } & \text { coded } \\
\text { MCSK } & \end{array}$ & $\begin{array}{l}\text { Concentration difference detector } \\
\text { based on i) Difference within a bit- } \\
\text { interval, ii) Difference in successive } \\
\text { bit-intervals }\end{array}$ & Sbs & Non-coherent & Low \\
\hline [137] & OOK & $\begin{array}{l}\text { Single sample detector with initial } \\
\text { distance estimation based on pilot } \\
\text { signal }\end{array}$ & Sbs & Coherent & Very high \\
\hline [119] & OOK & $\begin{array}{l}\text { Convexity detector with detection } \\
\text { threshold as weighted sum of earlier } \\
\text { convexity metrics }\end{array}$ & Sbs & Non-coherent & High \\
\hline [151] & OOK & $\begin{array}{l}\text { Cooperative detection with OR/AND } \\
\text { fusion rules at FC }\end{array}$ & Sbs & Coherent & High \\
\hline [152] & OOK & Log LRT & Sbs & Coherent & High \\
\hline [121] & $\begin{array}{l}\text { OOK, } \\
\text { Depleted- } \\
\text { MoSK } \\
\text { MoSK) }\end{array}$ & Maximum likelihood detection & Sbs & Coherent & Moderate \\
\hline
\end{tabular}

mate of various parameters was also obtained. These parameters were related to resistance offered to the motion of nano-machines, noise effects on the motion and the impact of gradient of attractants' concentration.

\subsubsection{Relay-assisted-based mobile MC systems}

In [152], a decode-and-forward relaying scheme was proposed for MMC in the presence of drift. In this work, Log LRT was used at the receiver and relay to determine the detection threshold. Also, an optimal number of transmitted molecules at each of the transmitting nodes was found to minimize the end-to-end error probability. A relay-assisted MMC in the presence of drift was studied in [156]. In this work, optimal LRT-based detection was performed at the receiver in each time slot. Further, for performance analysis through the analytical framework, a closed-form expression for channel capacity was also derived, where a decrease in capacity was reported with an increase in interference due to other sources of transmission and due to an increase in the diffusion coefficient of the receiver. 
Table 6 - BER performance of different detection schemes

\begin{tabular}{|c|c|c|c|c|c|}
\hline Reference & Modulation & Detection & Lowest BER & $\begin{array}{l}\text { SNR/Number } \\
\text { of transmitted } \\
\text { molecules }\end{array}$ & $\begin{array}{l}\text { Distance } \\
\text { between } \\
\text { transmitter } \\
\text { and receiver }\end{array}$ \\
\hline [33] & MoSK & $\begin{array}{l}\text { i) MAP detection without ISI } \\
\text { ii) Viterbi detection with ISI }\end{array}$ & $\begin{array}{l}\text { i) } 5 \times 10^{-3} \\
\text { ii) } \approx 10^{-5}\end{array}$ & $4 \mathrm{~dB} / 10^{3}$ & $100 \mu \mathrm{m}$ \\
\hline [34] & MoSK & $\begin{array}{l}\text { MAP detection with noise whitening } \\
\text { filter }\end{array}$ & $7 \times 10^{-6}$ & $10 \mathrm{~dB} / 2.5 \times 10^{4}$ & $600 \mu \mathrm{m}$ \\
\hline [40] & $\begin{array}{l}\text { Rectangular } \\
\text { pulse based } \\
\text { OOK }\end{array}$ & $\begin{array}{l}\text { Adaptive receiver based on steep- } \\
\text { est descent algorithm for i) Maxi- } \\
\text { mum likelihood sequence detection } \\
\text { ii) MMSE equalizer iii) DFE with de- } \\
\text { cision metric iv) DFE with quantizer }\end{array}$ & $\begin{array}{l}\text { i) } 0 \\
\text { ii) } 3 \times 10^{-5} \\
\text { iii) } 10^{-6} \\
\text { iv) } 3 \times 10^{-6}\end{array}$ & $16 \mathrm{~dB} / 4 \times 10^{9}$ & $0.05 \mu \mathrm{m}$ \\
\hline [45] & MTSK & $\begin{array}{l}\text { i) MMSE equalizer } \\
\text { ii) DFF }\end{array}$ & $\begin{array}{l}\text { i) } \approx 10^{-4} \\
\text { ii) } 3 \times 10^{-4}\end{array}$ & 500 & $5 \mu \mathrm{m}$ \\
\hline [52] & $\begin{array}{l}\text { Rectangular } \\
\text { pulsed based } \\
\text { OOK }\end{array}$ & $\begin{array}{l}\text { Non linear adaptive threshold detec- } \\
\text { tion based on local geometry and en- } \\
\text { ergy difference of received signal }\end{array}$ & $2 \times 10^{-4}$ & $8 \mathrm{~dB}$ & $9 \mathrm{~nm}$ \\
\hline [60] & $\begin{array}{l}\text { Rectangular } \\
\text { pulsed based } \\
\text { OOK }\end{array}$ & $\begin{array}{l}\text { Two layered detection for reduc- } \\
\text { ing the number of sequences to be } \\
\text { searched }\end{array}$ & $10^{-5}$ & $9 \mathrm{~dB} / 10^{4}$ & $3 \mu \mathrm{m}$ \\
\hline [29] & OOK & ANN detection with LM optimizer & $\approx 10^{-4}$ & $31 \mathrm{~dB}$ & $0.5 \mu \mathrm{m}$ \\
\hline [105] & OOK & MF detector & $2 \times 10^{-4}$ & $4 \times 10^{4}$ & $0.5 \mu \mathrm{m}$ \\
\hline [30] & OOK & Parzen-PNN based detection & $5 \times 10^{-4}$ & $10 \mathrm{~dB}$ & $2 \mu \mathrm{m}$ \\
\hline [111] & CSK & Fuzzy C-means clustering & $\approx 10^{-3}$ & $11 \mathrm{~dB} / 3 \times 10^{4}$ & $0.5 \mu \mathrm{m}$ \\
\hline [135] & OOK & Local convexity detection & $2 \times 10^{-4}$ & $31 \mathrm{~dB}$ & $0.065 \mu \mathrm{m}$ \\
\hline [31] & $\begin{array}{l}\text { Binary and M- } \\
\text { ary amplitude } \\
\text { modulation }\end{array}$ & $\begin{array}{l}\text { Recurrent neural network detection } \\
\text { schemes }\end{array}$ & $5 \times 10^{-4}$ & - & - \\
\hline [137] & OOK & $\begin{array}{l}\text { Single sample detector with initial } \\
\text { distance estimation based on pilot } \\
\text { signal }\end{array}$ & $4 \times 10^{-3}$ & $\begin{array}{l}3 \times 10^{4} \text { for pilot } \\
\text { signal and } 4 \times 10^{4} \\
\text { for information }\end{array}$ & $1 \mu \mathrm{m}$ \\
\hline
\end{tabular}

\subsubsection{Distribution detection-based mobile $M C$ systems}

In [157], 1-D MMC with drift was considered, where multiple cooperative nano-machines were used between the source and the destination. Channel capacity and the probability of bit error expressions were derived at the destination using the probabilities of detection and false alarm. Authors' analysis demonstrated that the optimal decision threshold at the destination depends on the probabilities of detection and false alarm of the last cooperative nano-machine.

A cooperative detection strategy was presented in [151], where several cooperative nano-machines sent their decisions to an FC about the presence or absence of an abnormality inside the blood vessel. In this work, each of the nano-machines and the FC were assumed to be mobile under the influence of both diffusion and drift. For performance analysis, the PDF of the first hitting time of molecule at the FC was also described, where the concept of effective diffusion coefficient was used as described in [117]. OR (if one of the nano-machines sent a positive report) and AND (if all the nano-machines sent a positive report) rules were used at the FC for making the global decision about the presence or absence of abnormality.
Authors demonstrated that for a higher probability of false alarm, OR rule performed better than AND rule and vice-versa. This is because in case of high chances of error, all the nano-machines may not be able to make the correct decision, and hence performance of the AND rule degrades. Further, to reduce the error probability, a cooperative abnormality detection scheme was proposed in [158]. In this scheme, a sensor could be activated if it detects an abnormality itself or it receives signaling molecules from other sensors that detected abnormality. Finally, an FC collected responses from all the sensors and checked the activation flag of all the sensors to decide the presence or absence of abnormality. Optimal threshold at the FC was derived by minimizing the error probability.

\subsection{Performance and complexity comparison of different detection techniques for mobile MC}

Computational complexity of the non-coherent detector proposed in [145] is $O\left(N_{s}\right)$ that is much less than the detection schemes such as coherent MAP method and MMSE [40], [98]. 
The complexity of the local convexity detector proposed in [135] is given by $O(l)+O\left(l^{2}\right)$, where $l$ is the length of a convexity metric. $O(l)$ is due to the calculations of the convexity metric and the threshold, $O\left(l^{2}\right)$ is the complexity due to the moving average operation. Further, the computational complexity of maximizing the likelihood in [137] was $O(\log (N))$. This was based on the Newton-Raphson method. This technique for detection was less complex than [118] (complexity is $O\left(S^{3}\right)$ ), as the CIR reconstruction and threshold determination in every bit interval was not required for detection. Instead authors in [137] used statistical characteristics of the CIR to estimate the initial distance and set the threshold for all bit intervals in advance for detection. Furthermore, the complexity in [119] was $O(S)$ that is also less than the technique presented in [118].

In [148], ZF-DFE and MMSE-DFE have computational complexity of $O\left(B M n_{t}^{2}\right)$, where $B$ is the block length, $M$ is the number of channel taps in an $n_{t} \times n_{t}$ MIMO system. Whereas a least squares DFE detector has a complexity of $O\left(B M 2^{n_{t}}\right)$. Thus, a least squares DFE detector has higher complexity but better BER performance than ZFDFE and MMSE-DFE. If channel memory is denoted as $M$, $n$ denotes the length of the sequence to be decoded, $L$ is the window length of SBRNN. $N$ is the number of states with highest log-likelihood values among $2^{M}$ states that are kept at each time instance in the beam search Viterbi algorithm. Then, as discussed in [31], $N=2^{M}$. Computational complexities of the Viterbi detector, RNN and SBRNN are given by $O(N n), O(n)$, and $O(L(n-L+1))$, respectively. It can be observed that $\mathrm{RNN}$ is most efficient in terms of computational complexity. SBRNN and beam search VD can have similar complexity. Complexity of traditional VD grows exponentially with memory length $M$.

The ANN detector proposed in [29] is less complex than the ANN-based detector in [31] as the number of hidden layers' neurons in the former technique are significantly less compared to the latter technique. Further, DFF proposed in [45] has a complexity of $O(M)$ whereas MMSE equalizer has a complexity of $O\left(M^{3}\right)$. Table 5 summarizes the modulation and detection techniques in $\mathrm{MMC}$ with or without drift in the channel. Further, Table 6 shows the lowest BER obtained in different detection schemes at a particular SNR. The distance between the transmitter and the receiver is also mentioned.

\subsection{Challenges in detection and possible solu- tions}

\subsubsection{Tracking the dynamic distance and deter- mining the adaptive threshold}

It can be seen from (7) and (8) that both peak time and peak amplitude depend on the distance between the transmitter and the receiver. One can also notice in Fig. 19 that the peak time decreases and peak amplitude in-

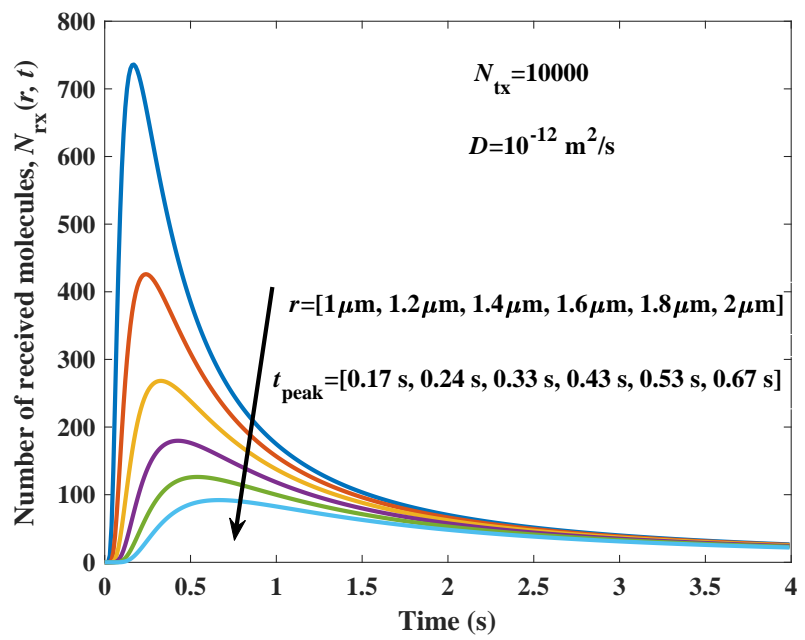

Fig. 19 - Comparison of the received signal without noise at different distances between the transmitter and the receiver. Respective peak times are also shown

creases as the distance between communicating nanomachines decreases. When the distance is $1 \mu \mathrm{m}$, the peak time and peak amplitude are around $0.017 \mathrm{~s}$ and 735 , respectively. However, when the distance is $2 \mu \mathrm{m}$, the peak time and amplitude are around $0.675 \mathrm{~s}$ and 92 , respectively. Hence, sampling at a fixed time (c.f. (7)) [117] is not suitable for detection under varying (or dynamic) distance over time, which arises due to mobility. For example, sampling at $t_{\text {peak }}$ in case of $r=1 \mu \mathrm{m}$ gives maximum signal but amplitude of signal becomes very less at the same time for $r=2 \mu \mathrm{m}$, as shown in Fig. 19. The impact of distance on the received signal (perturbed by the noise and ISI) has been shown in Fig. 20. The received signal is given by

$$
y(r(t), t)=\sum_{j=0}^{\infty} b_{j} N_{r x}\left(r(t), t-j T_{b}\right)+n(r(t), t) .
$$

In (18), the distance $r(t)$ is assumed to be time-varying, unlike (11) where distance $r(t)=r \forall t$ is constant. For this simulation, the transmitted sequence is considered as [ $\left[\begin{array}{lllll}1 & 1 & 0 & 1 & 0\end{array}\right]$ and the distance in each bit-interval is progressively increasing. It can be observed that in the 4 th bit-interval, the received signal for the transmitted bit-1 goes below the threshold, that causes incorrect detection. Hence, the threshold at the receiver should not be fixed (as considered in static MC). To address this issue, the decision threshold should be adaptive and dependent upon the dynamic distance. Moreover, for detection, the estimation of distance at the receiver under static and mobile conditions is also an important issue, which has been addressed in some of the works such as [118], [136], [137], [159], [160].

Under a mobility condition, as described in [135], the detection based on local convexity of the received signal (in case of bit-1 transmission) can be used since the convexity persists even if the peak time and peak amplitude change (c.f. Fig. 19). 


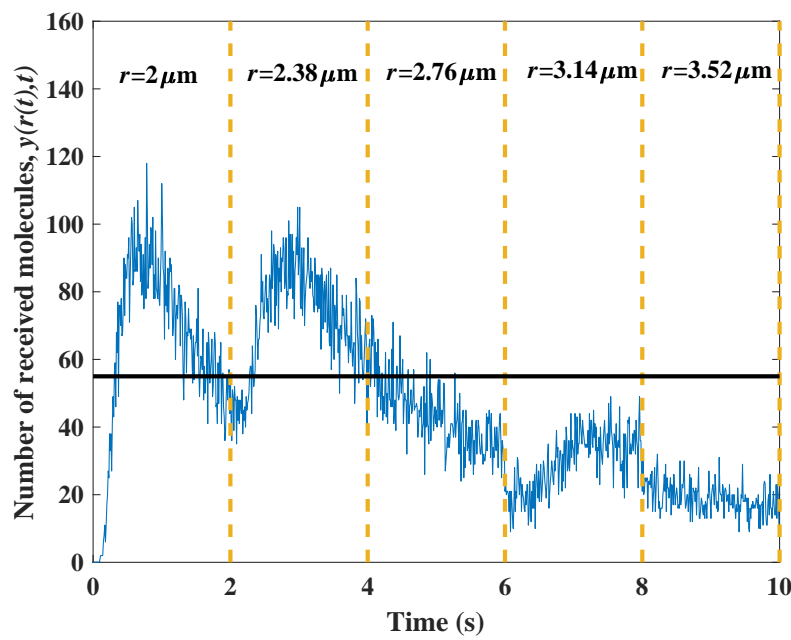

Fig. 20 - Received signal including noise and ISI for the transmitted sequence $\left[\begin{array}{lllll}1 & 1 & 0 & 1 & 0\end{array}\right]$. The maximum signal is below the threshold in 4 th bit duration for bit-1 leading to incorrect detection.

The detection based on the convexity metric is also suitable for the system experiencing only ISI, where noise can be filtered by using a moving average filter as described in [135]. Further, to improve the performance, the ISI mitigation technique described in [118] can be considered under mobile scenarios. In this work, dynamic distance is estimated using (6) if the number of received molecules $N_{\text {rx }}(r, t)$ is known. This estimated distance is further used to reconstruct the received signal (within a bit-interval) and subtract it from the total received signal for ISI mitigation. Note that the distance estimation using (6) in [118] is not correct if bit-0 is transmitted as $N_{\text {tx }}$ is zero for bit- 0 .

Hence, in [118], the adaptive threshold for detection in the current bit-interval was calculated using the distance estimated in the bit-interval when the last bit- 1 was detected. If the coherence time of the channel is small then the channel can change considerably between the current bitinterval and the bit-interval in which the last bit-1 was detected. Hence, the detection threshold can be wrong. This problem can be solved by transmitting some molecules for bit-0, as well [136]. Considering the non-zero number of molecules for bit-0, the adaptive threshold in the current bit-interval can be obtained using the distance estimated in the previous bit-interval. Also, for MMC in a drift-based environment, threshold determination has been shown in [152].

\subsubsection{Multiple parameters estimation}

If the number of transmitted molecules $N_{\text {tx }}$ and the diffusion coefficient $D$ change along with the distance $r$ then the estimate of these parameters over time is difficult to obtain using (6). In [161], a method for estimating multiple parameters has been shown. If we consider that $N_{\mathrm{tx}}$, $D$, and $r$ are constant within a bit-interval then three samples of the received signal within a bit-interval will be re- quired as there are three unknown variables to be determined. By solving three different equations similar to (6) with three samples of the received signal $N_{\mathrm{rx}_{1}}, N_{\mathrm{rx}_{2}}$, and $N_{\mathrm{rx}_{3}}$, three unknown parameters i.e., $N_{\mathrm{tx}}, D, r$ can be estimated. However, for a mobile transmitter and receiver, as the distance $r$ changes at each sampling time, we cannot estimate five unknown variables i.e., $N_{\mathrm{tx}}, D, r_{1}, r_{2}, r_{3}$ using three samples of the received signal $N_{\mathrm{rx}_{1}}, N_{\mathrm{rx}_{2}}$, and $N_{\mathrm{rx}_{3}}$. Hence, non-coherent detectors [119] and ANN detectors [31] are more suitable in this case.

\subsubsection{Unknown channel model}

If channel model is not known then the adaptive threshold-based detection techniques used in [118] and [136] will not yield good performance because (6) can not be used for distance estimation. Therefore, to deal with unknown channel model, non-coherent detection techniques proposed in [145], [146], [119] can be considered. In [146], amplitude difference was used as the decision metric, whereas energy difference was used as the decision metric in [145]. Also, the technique proposed in [145] is suitable for the scenario when the system experiences strong ISI. Further, to improve the detection performance in noisy and unknown channel scenarios, ANN detectors [31] can also be considered as one of the possible solutions.

\subsubsection{Synchronization}

For a static MC scenario, few synchronization techniques such as blind synchronization [161], [162], synchronization using peak observation time and threshold triggering [163], and reference broadcast synchronization considering molecule synthesis time [164] have been proposed. Note that these synchronization schemes proposed for static MC cannot be used in MMC scenarios. Moreover, synchronization is more challenging in mobile MC due to the time-varying distance between the communicating nano-machines. It is also worth noting that joint detection and synchronization have to be done to realize practical mobile MC systems. Recently, two synchronization schemes based on the least-square method and peak time have been proposed for mobile MC in [165]. However, these techniques rely on a known channel model and will not work if extended for unknown channel models e.g., channel model with multiple fully-absorbing receiver nano-machines. Considering limited computational resources for nano-machines, investigation of novel asynchronous detection techniques for mobile MC is required. 


\section{EXPERIMENTAL WORKS}

\subsection{Microscale experiments}

A microfluidic chip for detecting a Deoxyribonucleic acid (DNA) molecule was proposed in [166]. This chip was fabricated using multilayer soft lithography, which consists of two layers of silicone rubber i.e., Polydimethylsiloxane (PDMS), a flow layer with several micro-wells, and a control layer. Possibilities of nano-resonators made of Graphene sheets and Carbon Nano-Tubes (CNTs) for transportation and detection of molecules at very low concentration were explored in [167]. These materials detect the shift in wave velocity (inside CNT) and resonant frequencies due to the presence of external molecules. The motion of water molecules inside the CNT channel was demonstrated in this work. Moreover, nano-pumping action to initiate the molecule flow was also shown. This setup could be helpful for the design of transmitters in MC.

Silicon-Nano-Wire (Si-NW)-based biomolecule detection was presented in [168]. In [168], a faster response of Si-NW than the Ion-Sensitive Field Effect Transistor (ISFET) was shown. Also, the sensitivity of the Si-NW sensor was revealed to be dependent upon the $\mathrm{pH}$ level of the surroundings, the geometry of the sensor, and its doping level. In [169], detection of femtograms of mercury-ion was proposed using Bovine Serum Albumin (BSA) protein conjugated $\mathrm{ZnO}$ nano-sphere (BCZ). In this work, a logarithmic variation of conductance of BCZ was demonstrated with the concentration of mercury-ions. Further, the formation of a complex between BCZ and mercury was confirmed through fluorescence spectroscopy. Note that the proposed work in [169] has several biomedical applications such as detection of diseases related to ingestion of mercury and other heavy metals, which are toxic for a human body.

A microscale modulator was proposed in [170], which converted an optical signal from a Light-Emitting Diode (LED) to a chemical signal consisting of protons. The proposed system was similar to one considered in [171], where incident light on bacteria released the $\mathrm{H}^{+}$ions. On the other hand, a pH sensor was employed at the receiver. For this system, channel estimation schemes based on short-duration pilot signals and long duration previously detected data were proposed. Moreover, the optimal maximum likelihood detector and suboptimal derivativebased detector were also derived. Simulation results showed that the system can achieve a bit rate of 1 bit per minute. A Graphene-based micro-resonator for individual molecule detection of DNA, dopamine, and nicotine was proposed in [172]. An ingestible pill with the bidirectional wireless interface was proposed in [173] for inbody nucleic acid-sensing. In this work, transmit and received powers of the order of micro-watts was demonstrated. Also, a receiver mode sensitivity of $-59 \mathrm{dBm}$ with BER of $10^{-3}$ was shown at the speed of 1 megabits per second (Mbps).

\subsection{Macroscale experiments}

An experimental setup was presented in [174] to model the diffusive channel. In this setup, a spray of isopropyl alcohol was used as a transmitter and a metal oxide sensor was used as a receiver that generates a voltage based on the concentration of alcohol received over the air. The distance between the transmitter and the receiver was considered from 2 to 5 meters and spraying duration was also varied from 50 milliseconds to 200 milliseconds under different trials. Moreover, the response of the receiver was averaged over these trials and the unknown coefficients in the diffusion equation were found by minimizing the difference between the experimental observations and the theoretically expected response.

A $2 \times 2$ MIMO-MC system was developed in [175] and [176], where two alcohol sprays and two chemical sensors were used at the transmitter and receiver, respectively. In this work, a micro-controller for transmission and two micro-controllers for decoding the text message was used. Simulation results showed a data rate improvement of 1.7 times with respect to the SISO system. Signal-to-inter link interference of 14.567 was calculated using the MIMO test bed when the transmitter and receiver were separated by $90 \mathrm{~cm}$ and antenna separation was $40 \mathrm{~cm}$. Such communication systems could be useful for structural high speed health monitoring for smart cities and also for the transmission of signals to robots in a subterranean (i.e, under the earth surface) region where implementing radio frequency communication is challenging.

An experimental setup was demonstrated in [177] in which a peristaltic pump is used as a transmitter and a $\mathrm{pH}$ sensor is employed at the receiver. The peristaltic pump used acid to transmit bit- 0 and base to transmit bit-1 over the channel which contains water in a silicon tube. In the case of an acidic signal, the receiver generated positive voltage and for the base signal, the receiver generated negative voltage. For detection, two different techniques were proposed in which Support Vector Machine (SVM) and RNN are used. The SVM-based detection scheme uses a linear relationship between the input and the output. Also, a slope-based detector was used which measures the rate of change of $\mathrm{pH}$ within a symbol interval. It was shown that $\mathrm{RNN}^{13}$ performs better among other detectors for a short symbol duration. On the other hand, slope-based detection performed the worst. Hence, training based detectors are suitable for cases where the channel model is not known.

In [178], the authors used a test bed that was similar to [174]. In this setup, isopropyl alcohol spray and metal oxide sensor was used for transmission and reception,

\footnotetext{
${ }^{13} \mathrm{RNN}$ was trained using the slope values and corresponding bit patterns.
} 
respectively. At the transmitter, to control the function (turn on/off spray), an Arduino Uno microprocessor was connected. A computer was used for uploading the control codes to the microprocessor. On the other hand, the receiver consisted of an MQ-3 semiconductor metal oxide gas sensor and a microprocessor, which was used to read the sensor data. Finally, for decoding the transmitted information, the detection algorithm was implemented on the computer connected to the microprocessor. A modified slope-based detection was proposed in [178] in which the number of positive and negative slope values within a bit duration was counted to decide in favor of bit- 1 or bit- 0 . It was shown that in the case of bit- 1 , $80 \%$ of the slope values were positive. In this work, the number of samples within a bit duration was set as 10 and the detection threshold was considered as 8 based on $80 \%$ criterion.

An experimental MMC system was proposed in [179] in which the mobile receiver was able to sense the gradient of alcohol molecules formed in the environment. This gradient was formed by spraying the molecules by the target. The mobile receiver was a two-wheeled vehicle controlled by an Arduino-Uno microprocessor. A metal oxide sensor was mounted on the vehicle to sense the concentration of alcohol molecules. A bio-inspired algorithm similar to bacterial chemotaxis was used by the receiver to move towards the target. It was shown that this algorithm was more efficient than a random walk process in terms of the time required to reach the target.

In [180], an ethanol electrical sprayer was used as a transmitter. To create a flow-based channel, a table fan was used to provide velocity to the transmitted molecules. Moreover, at the receiver, the detection was based on an adaptive threshold mechanism where the decision threshold was calculated using the first sample of each time slot. The proposed detection scheme was claimed to be better than the one proposed in [178], which obtains the decision threshold in terms of the number of positive slope values of the received signal. A test bed based on proton pumping bacteria was proposed in [171], where incident light on a plasmid inside bacteria generated protons which were later sensed by a $\mathrm{pH}$ sensor at the receiver.

An experimental setup for validating the theoretical models was proposed in [181]. In this work, Hydrochloric acid $(\mathrm{HCl})$ controlled by a solenoid valve was used as the transmitter and a pH sensor was deployed as the receiver. Moreover, a glass pipe containing water was considered as the channel for communication between the transmitter and the receiver. At the receiver, adaptive thresholdbased detection was performed considering the channel memory of the previous one bit or two bits. Interestingly, COMSOL simulations of the experimental setup were also carried out to compare against the experimental data. An experiment similar to [174] was also carried out in [182]. However, in this work, multilevel modulation was pro- posed where different symbols were sent by varying the time duration of releasing the molecules. A slope-based detection along with experimentally validated channel and noise models were also presented. A test bed using SPIONs as the information particles was proposed in [183]. In this work, SPIONs were sent into a pipe containing the water, and a susceptometer was used for detecting the particles. The change in inductance was investigated under the various distribution of particles passing. A MAP demodulator based on chemical reactions has been presented in [184]. It was shown that a gene promoter circuit DCS2 found in yeast can be used for MAP-based demodulation.

A transmitter using acetone and methanol for signaling with $N_{2}$ gas as a carrier and a mass spectrometer with a quadrupole mass analyzer as detector were used in [185]. Tangential and longitudinal diffusion coefficients were taken into account for the analysis of amplitude, energy, and SNR of the received signal. An implantable glucose sensor was proposed in [186] in which InGaZnO (IGZO)-based Electrolyte-Gated Field-Effect Transistors (EGFETs) were considered as the sensor material. This material has better field-effect mobility and a large on-off ratio than previously used materials e.g., Graphene and SiNW. The basic principle of sensing in [186] was to sense the $\mathrm{pH}$ value due to the $H^{+}$ions generated by the reaction of glucose (to be sensed) and glucose oxidase (i.e., the material immobilized on the sensor). A test bed showing its effectiveness was also implemented. Also, RNN and module-based decoding where each module consists of ANN were used for the detection of $\mathrm{pH}$ value. Simulation results demonstrated that deep learning-based algorithms achieve better BER performance in unknown and noisy channels.

In [187] a channel coding scheme was proposed to mitigate ISI. In the codeword, the following three properties were used: i) Sending consecutive bit- 1 was not allowed, ii) The codeword always started with bit- 0 , and iii) At-least one bit-1 should be present in each codeword. Also, an adaptive threshold was used to decode each codeword, where the threshold scaling factor was estimated by sending the pilot signal before information transmission. The proposed technique was also tested experimentally where alcohol spray and alcohol sensor was employed as the transmitter and receiver, respectively. Authors demonstrated that $\mathrm{BER} \approx$ $5 \times 10^{-2}$ can be obtained with this setup. Particle image velocimetry and planar laser-induced fluorescence were proposed in [188] for tracking the changes in molecular signals as well as the channel parameters responsible for a change in the molecular signal.

A cooperative RNN was proposed in [189] in which RNN had forward and backward components. A merged layer with the time-varying property was designed to combine the outputs of forward and backward RNN components. The proposed network was tested experimentally on a 
test bed structure similar to [177]. The BER in training and testing data sets was $\approx 0.032$ and $\approx 0.074$, respectively. The proposed network achieved BER less than SBRNN and a simple detector, where $\mathrm{pH}$ difference of last and first values within a bit-interval was used as the decision metric. Table 7 shows a brief description of the experimental works and concluding remarks on the outcomes of the experiments.

\section{CHALLENGES IN PRACTICAL DESIGN OF TRANSMITTER AND RECEIVER, AND FUTURE RESEARCH DIRECTIONS}

\subsection{Transmitter design}

Most of the existing works consider the transmitter to be an ideal point source that can release molecules in the direction of the receiver. This is a very weak assumption, which has been employed for analytical tractability to analyze the system performance. However, for realizing a practical spherical transmitter, the following issues must be taken into consideration:

\subsubsection{Energy}

The transmitter requires some energy for its operation for example encoding the transmitted information, controlling the number of emitted molecules, etc. Therefore, it should have the capability of harvesting energy. For health applications within IoBNT, this energy harvesting can be done by using the chemical molecules present in the environment. For example, glucose is the natural source of energy in all organisms. Similarly, the transmitter can also use some chemical to energize itself.

\subsubsection{Molecule synthesis}

The transmitter is expected to release the signaling molecules to communicate with the receiver. Hence, the very first requirement is the synthesis of molecules in the required amount. To achieve this, a controlled chemical reaction is required. On the other hand, the signaling molecules/drug particles can also be loaded beforehand so that there is no requirement for molecule synthesis. Thus, for biomedical applications e.g., drug delivery, the required number of chemical molecules should be calculated before inserting the nano-machine inside the human body.

\subsubsection{Modulator}

As discussed in the previous sections, various types of modulation schemes have been proposed in the literature, e.g., to transmit information, the number of released molecules is varied, or the release time of the molecules is varied. Hence, a unit at the transmitter is required to control the concentration or release time of the molecules.

\subsubsection{Molecule release mechanism}

The transmission of molecules requires a pumping action. Therefore, nano-pumps, as described in [167], can be used for this purpose. Moreover, by applying mechanical loads to the CNTs, molecular flows can also be achieved.

\subsubsection{Biocompatibility}

Since these nano-machines are expected to perform their task inside the human body, they should be biocompatible and should not produce any immunological response or toxic effects while performing their task in an environment of living cells. For example, a biocompatible polymer like chitosan conjugated with anticancer agents is used for gradual drug release in cancerous tissues. Ensuring biocompatibility requires interdisciplinary research.

\subsection{Receiver design}

The types of receiver considered in the literature are mostly passive receiver, absorbing receiver, and reactive receiver. Passive receivers are assumed to be a hypothetical sphere transparent to the arriving molecules. This implies that the passive receiver can count the number of molecules within its closed boundary without affecting their propagation in the environment. In contrast to the passive receiver, an absorbing receiver can count and absorb the molecules once they hit the surface. On the other hand, reactive ${ }^{14}$ receivers have receptors on their surface that can bind to the molecules through a reversible reaction. In such a scenario, counting the number of bound receptors can provide the information of received molecules.

Note that passive and absorbing receivers are difficult to develop in practice. Thus, a reactive receiver can be considered for the practical design of the receiver. It is also important to note that the functioning of the reactive receiver is similar to the cells which can bind respective ligands and provide transduction of extracellular signals to intracellular signals. Bio-FET can also be used as a receiver to realize MC.

\subsubsection{Receptor design}

Since the receiver is expected to bind the signaling molecules at its receptor, the design of receptors is important. The selectivity and sensitivity of the receptors to the intended molecule can be done by selecting the proper material for the receptor. Selectivity means the receptor should bind only that molecule, which is used for signaling. Sensitivity means the response time of the receptors. The response time should not be very high otherwise it will limit the data rate of the MC system. As discussed in [18], aptamers and DNAs can be appropriate to be used as receptors. Various types of aptamers and corresponding ligand/signaling molecules are available [190], [191], [192].

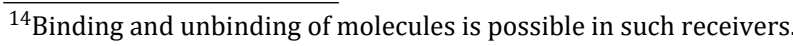


Table 7 - System description and important conclusions in experimental works

\begin{tabular}{|c|c|c|}
\hline Reference & System description & Conclusion \\
\hline [168] & Silicon-nano wire (Si-NW) based biomolecule detection & $\begin{array}{l}\text { Faster response of Si-NW than the Ion-Sensitive } \\
\text { Field-Effect Transistor (ISFET) was shown. }\end{array}$ \\
\hline [169] & $\begin{array}{l}\text { Detection of femtograms of Mercury-ion using BSA protein } \\
\text { conjugated } \mathrm{ZnO} \text { nano-sphere (BCZ) }\end{array}$ & $\begin{array}{l}\text { i) Logarithmic variation of conductance of BCZ with } \\
\text { the concentration of Mercury-ions. } \\
\text { ii) Useful for biomedical applications such as detec- } \\
\text { tion of toxic heavy metals in body. }\end{array}$ \\
\hline [170] & $\begin{array}{l}\text { i) Conversion of optical signal from LED to chemical signal } \\
\left(\mathrm{H}^{+} \text {ions }\right) \\
\text { ii) Detection of } \mathrm{H}^{+} \text {ions using pH sensor }\end{array}$ & $\begin{array}{l}\text { i) Derivative-based detection was used to obtain a bit } \\
\text { rate of } 1 \text {-bit/min. } \\
\text { ii) System can work as microscale modulator. }\end{array}$ \\
\hline$[174]$ & $\begin{array}{l}\text { i) Transmitter - Spray of isopropyl alcohol } \\
\text { ii) Channel - Air } \\
\text { iii) Receiver - Metal oxide sensor }\end{array}$ & $\begin{array}{l}\text { i) Study of diffusion over the air } \\
\text { ii) Determines and validates the channel model ex- } \\
\text { perimentally. }\end{array}$ \\
\hline [176] & $\begin{array}{l}\text { i) } 2 \times 2 \text { MIMO-MC system using two alcohol sprays and two } \\
\text { chemical sensors }\end{array}$ & $\begin{array}{l}\text { i) } 1.7 \text { times data rate improvement w.r.t. SISO sys- } \\
\text { tem. } \\
\text { ii) Useful for applications like structural high speed } \\
\text { health monitoring in smart cities. }\end{array}$ \\
\hline$[177]$ & $\begin{array}{l}\text { i) Transmitter - Peristaltic pump using acid to transmit bit- } \\
0 \text { and base to transmit bit-1 } \\
\text { ii) Channel - Water in a silicon tube } \\
\text { iii) Receiver- RNN-based detection }\end{array}$ & $\begin{array}{l}\text { Training-based detectors are suitable if channel } \\
\text { model is not known. }\end{array}$ \\
\hline [179] & $\begin{array}{l}\text { i) Transmitter - Alcohol spray } \\
\text { ii) Channel - Air } \\
\text { iii) Mobile receiver - Metal oxide sensor mounted on a ve- } \\
\text { hicle }\end{array}$ & $\begin{array}{l}\text { Better target (transmitter) detection by the receiver } \\
\text { using bio-inspired algorithm than the random walk } \\
\text { process. }\end{array}$ \\
\hline [186] & $\begin{array}{l}\text { i) Implantable glucose sensor is proposed } \\
\text { ii) InGaZnO-based EGFET is used as the sensor material } \\
\text { iii) Detection of } H^{+} \text {ions generated by the reaction of glucose } \\
\text { using RNN }\end{array}$ & $\begin{array}{l}\text { i) Deep learning-based detection performs better } \\
\text { than conventional threshold based detectors in un- } \\
\text { known and noisy channels. } \\
\text { ii) EGFETs have better field-effect mobility and a } \\
\text { large on-off ratio than Si-NW. }\end{array}$ \\
\hline [187] & $\begin{array}{l}\text { i) Transmitter - Alcohol spray } \\
\text { ii) Channel - Air } \\
\text { iii) Receiver- Alcohol sensor }\end{array}$ & $\begin{array}{l}\text { i) Channel coding for better ISI mitigation. } \\
\text { ii) Adaptive threshold for decoding of the code-word. }\end{array}$ \\
\hline [189] & $\begin{array}{l}\text { i) Transmitter - Peristaltic pump using acid to transmit bit- } \\
0 \text { and base to transmit bit-1 } \\
\text { ii) Channel - Water in a silicon tube } \\
\text { iii) Receiver- Cooperative RNN-based detection }\end{array}$ & Better BER performance than SBRNN. \\
\hline
\end{tabular}

\subsubsection{Transduction unit}

In bio-FET, the channel between drain and source electrodes is the transduction unit for generating an electrical signal based on the molecular signal at the receptors. Single-Walled CNTs (SWCNTs), Si-NW [168], graphene [167] could be used as the transduction unit. The SWCNT for Acetylcholine (Ach) receptor has been discussed in [193] to detect Ach. Si-NW-based transduction unit for re- ceptor protein in prostate cancer detection has been pre- sented in [194]. The graphene-based transducer channel for a glucose oxidase receptor in a glucose sensing device has been discussed in [195]. The conductivity of trans- ducers such as SWCNT, Si-NW and graphene varies with the number of molecules bound to the receptors. This in turn changes the current flow between source and drain of bio-FET [18] thus providing transduction capability to these materials. SWCNT and Si-NW are one dimensional structures but graphene is a two dimensional material that can provide large spatial coverage leading to an increase in the number of receptors that can be functionalized on its surface.

\subsubsection{Signal processing unit}

After the transduction unit, the processing of the generated electrical signal is required for the detection of information. Since a nano-machine is expected to be a simple device with limited computational capability, simple and less-complex detectors such as derivative-based detection [61], asynchronous detection [50] are more suitable. However, the limitation of these detectors is that they cannot perform well under high noise and mobile transmitter/receiver scenarios. Moreover, the non-coherent detector can also be used in challenging environments where the channel model is difficult to obtain. A noncoherent detector based on local convexity [135] that uses a moving average operation for noise reduction and ANN- 
based detection can be robust detectors [31] as they do not require CSI and are also capable of working in the high noise environment.

\subsection{Future research directions}

Within IoBNT, multiple transmitters and receivers have to work together to perform complex tasks, including sensing and actuation. However, exact analytical channel models considering multiple fully absorbing receivers in the medium are not available in the existing literature due to mathematical intractability of corresponding diffusion equations. In this context, the work in [196] considered two fully absorbing receivers in a 3-D medium and derived an approximate first hitting time distribution to demonstrate the mutual dependency between receivers. However, this approximation is only valid when $r_{1}>3 a$, $r_{2}>3 a$, and $R>3 a$, where $r_{1}, r_{2}, R$, and $a$ denote the distance between the transmitter and first receiver, distance between the transmitter and second receiver, distance between the first and second receiver, and the radius of the receiver, respectively. In this derivation, the radius of each receiver is assumed to be identical.

The analysis was further extended for an underlay-based cognitive paradigm in [197] where both primary and secondary link performances were evaluated by employing a simple molecule control mechanism at the secondary transmitter. In this work, the radius of each receiver is assumed to be different and the impact of molecule degradation over time was also considered while analyzing the system performance. However, the analyses in [196] and [197] is restricted only for two fully absorbing receivers and cannot be easily extended for more than two receivers. Thus, development of exact analytical channel models involving multiple fully absorbing receivers in a 3-D medium is still an open problem.

Further, many MC systems [33], [40], [45], [118], [148], [136], [144] that are proposed in the literature are coherent. These coherent MC systems require CSI and suffers from the drawback of complex channel estimation. Note that the channel in these systems can be very unpredictable with very short coherence times. Hence the MC systems that use pilot signal [137], [148], [187] for estimating the CSI, are not very suitable. Also, the receivers that can detect the information for fast-varying channels (i.e., short coherence time) are required to build practical MC systems. Machine/deep learning-based MC systems that do not need CSI and perform well in fastvarying channels [31] are suitable in such complex scenarios. Also, machine/deep learning-based algorithms do not rely on accurate channel models and can be classified as non-coherent schemes. Hence, the possibility of implementing these algorithms should be explored to address IoBNT applications with static [30], [186], [189] and mobile MC [106], [31].
In this context, one of the possible research areas is neural network detection considering the channel model which is not known or difficult to obtain. Further, the performance of different first order and second order algorithms [198] used for optimizing the neural network detectors should be investigated.

On the other hand, most of the detection schemes assume perfect synchronization between the transmitter and the receiver. However, for practical MC systems, joint synchronization and detection or asynchronous detection has to be performed, as shown in [163] and [50], respectively. In this context, novel low-complexity schemes should be devised to perform asynchronous detection or detection with synchronization at the fully absorbing receiver, especially for a flow-induced mobile MC where each of the nano-machines are considered to be mobile in diffusive channel along with drift. Moreover, the state of the art detectors [45], [52], [118], [146] considered perfect synchronization while analyzing the system performance. Thus, the performance evaluation of these detectors considering synchronization error is still lacking in the current literature.

\section{CONCLUSION}

For the IoBNT applications such as drug delivery, in-body health monitoring, etc, the nanoscale and microscale devices are expected to perform collaborative tasks using MC. However, the MC system performance in these applications significantly depends on the transmission and detection schemes employed at the transmitter and receiver nano-machines (or bio-nano-machines), respectively. This survey, therefore, presented the transmission and detection techniques existing in the current literature for static and mobile nano-machines under purediffusive and flow-induced diffusive channels. In each category, different types of MC system such as SISO, MIMO, relay-assisted, and FC-based cooperative detection scheme have been discussed to support several health applications within IoBNT. Various coherent and noncoherent detection schemes are presented under each category. The detectors have also been classified based on symbol-by-symbol detection and sequence detection. Also, the performance and the complexities of some detection techniques are discussed. Further, several challenges in detection have been described under various scenarios. Experimental works related to $\mathrm{MC}$ are also presented. At the end of this survey, some major challenges related to the practical design of the transmitter and receiver along with future research directions have been added. 


\section{REFERENCES}

[1] Tadashi Nakano, Andrew W Eckford, and Tokuko Haraguchi. Molecular communication. Cambridge University Press, 2013.

[2] Ian F Akyildiz, Max Pierobon, Sasi Balasubramaniam, and Y Koucheryavy. "The internet of bionano things". In: IEEE Communications Magazine 53.3 (2015), pp. 32-40.

[3] Young Il Kim, Yoonkwon Park, and Hongkoo Baik. "A novel detection for biomolecule using RF LC resonator". In: SENSORS, 2007 IEEE. IEEE. 2007, pp. 728-731.

[4] Puneet Manocha, Gitanjali Chandwani, and Soumen Das. "Characterization of Dielectrophoresis Based Relay-Assisted Molecular Communication Using Analogue Transmission Line". In: IEEE Access 8 (2020), pp. 33352-33359.

[5] Wei-Kang Hsu, Xiaojun Lin, and Mark R Bell. "Deep-target delivery of nanosensors with bacteria-inspired coordination". In: GLOBECOM 2017-2017 IEEE Global Communications Conference. IEEE. 2017, pp. 1-6.

[6] Muneer M Al-Zu'bi and Ananda Sanagavarapu Mohan. "Modelling of implantable drug delivery system in tumor microenvironment using molecular communication paradigm". In: IEEE Access 7 (2019), pp. 141929-141940.

[7] Ian F Akyildiz, Maysam Ghovanloo, Ulkuhan Guler, Tevhide Ozkaya-Ahmadov, A Fatih Sarioglu, and Bige D Unluturk. "PANACEA: An internet of bionanothings application for early detection and mitigation of infectious diseases". In: IEEE Access 8 (2020), pp. 140512-140523.

[8] Youssef Chahibi and Ilangko Balasingham. "An intra-body molecular communication networks framework for continuous health monitoring and diagnosis". In: 2015 37th Annual International Conference of the IEEE Engineering in Medicine and Biology Society (EMBC). IEEE. 2015, pp. 4077-4080.

[9] Luca Felicetti, Mauro Femminella, and Gianluca Reali. "A molecular communications system for live detection of hyperviscosity syndrome". In: IEEE Transactions on NanoBioscience (2020).

[10] Steven Kisseleff, Robert Schober, and Wolfgang $\mathrm{H}$ Gerstacker. "Magnetic nanoparticle based interface for molecular communication systems". In: IEEE Communications Letters 21.2 (2016), pp. 258-261.
[11] Federico Tavella, Alberto Giaretta, Triona-Marie Dooley-Cullinane, Mauro Conti, Lee Coffey, and Sasitharan Balasubramaniam. "DNA molecular storage system: Transferring digitally encoded information through bacterial nanonetworks". In: IEEE Transactions on Emerging Topics in Computing (2019).

[12] Tania Islam, Ethungshan Shitiri, and Ho-Shin Cho. "A Simultaneous Drug Release Scheme for Targeted Drug Delivery Using Molecular Communications". In: IEEE Access 8 (2020), pp. 91770-91778.

[13] Michael Taynnan Barros, Walisson Silva, and Carlos Danilo Miranda Regis. "The multi-scale impact of the Alzheimer's disease on the topology diversity of astrocytes molecular communications nanonetworks". In: IEEE Access 6 (2018), pp. 78904-78917.

[14] Ian F Akyildiz, Jiande Chen, Maysam Ghovanloo, Ulkuhan Guler, Tevhide Ozkaya-Ahmadov, Massimiliano Pierobon, A Fatih Sarioglu, and Bige D Unluturk. "Microbiome-gut-brain axis as a biomolecular communication network for the Internet of Bio-NanoThings". In: IEEE access 7 (2019), pp. 136161-136175.

[15] Reza Mosayebi, Arman Ahmadzadeh, Wayan Wicke, Vahid Jamali, Robert Schober, and Masoumeh Nasiri-Kenari. "Early cancer detection in blood vessels using mobile nanosensors". In: IEEE transactions on nanobioscience 18.2 (2018), pp. 103-116.

[16] Nariman Farsad, H Birkan Yilmaz, Andrew Eckford, Chan-Byoung Chae, and Weisi Guo. "A comprehensive survey of recent advancements in molecular communication". In: IEEE Communications Surveys \& Tutorials 18.3 (2016), pp. 1887-1919.

[17] Uche AK Chude-Okonkwo, Reza Malekian, Bodhaswar T Maharaj, and Athanasios V Vasilakos. "Molecular communication and nanonetwork for targeted drug delivery: A survey". In: IEEE Communications Surveys \& Tutorials 19.4 (2017), pp. 3046-3096.

[18] Murat Kuscu, Ergin Dinc, Bilgesu A Bilgin, Hamideh Ramezani, and Ozgur B Akan. "Transmitter and receiver architectures for molecular communications: A survey on physical design with modulation, coding, and detection techniques". In: Proceedings of the IEEE 107.7 (2019), pp. 1302-1341.

[19] Tadashi Nakano, Yutaka Okaie, Shouhei Kobayashi, Takahiro Hara, Yasushi Hiraoka, and Tokuko Haraguchi. "Methods and applications of mobile molecular communication". In: Proceedings of the IEEE 107.7 (2019), pp. 1442-1456. 
[20] V. Jamali, A. Ahmadzadeh, W. Wicke, A. Noel, and R. Schober. "Channel Modeling for Diffusive Molecular Communication-A Tutorial Review". In: Proceedings of the IEEE 107.7 (2019), pp. 1256-1301. DOI: 10 . 1109 / JPROC . 2019. 2919455.

[21] Christian A Söldner, Eileen Socher, Vahid Jamali, Wayan Wicke, Arman Ahmadzadeh, HansGeorg Breitinger, Andreas Burkovski, Kathrin Castiglione, Robert Schober, and Heinrich Sticht. "A survey of biological building blocks for synthetic molecular communication systems". In: IEEE Communications Surveys \& Tutorials (2020).

[22] M. Ş. Kuran, H. B. Yilmaz, I. Demirkol, N. Farsad, and A. Goldsmith. "A Survey on Modulation Techniques in Molecular Communication via Diffusion". In: IEEE Communications Surveys Tutorials 23.1 (2021), pp. 7-28. DOI: 10 . 1109 / COMST . 2020.3048099.

[23] Bhushan S Pattni and Vladimir P Torchilin. "Targeted drug delivery systems: Strategies and challenges". In: Targeted drug delivery: Concepts and design. Springer, 2015, pp. 3-38.

[24] Adam Noel, Karen C Cheung, and Robert Schober. "Improving receiver performance of diffusive molecular communication with enzymes". In: IEEE Transactions on NanoBioscience 13.1 (2014), pp. 31-43.

[25] Rakesh K Jain and Triantafyllos Stylianopoulos. "Delivering nanomedicine to solid tumors". In: $\mathrm{Na}$ ture reviews Clinical oncology 7.11 (2010), p. 653.

[26] Mauro Femminella, Gianluca Reali, and Athanasios V Vasilakos. "A molecular communications model for drug delivery". In: IEEE transactions on nanobioscience 14.8 (2015), pp. 935-945.

[27] Ghalib H Alshammri, Walid KM Ahmed, and Victor B Lawrence. "Adaptive Batch Training Rule-Based Detection Scheme for ON-OFF-Keying DiffusionBased Molecular Communications". In: 2018 IEEE 13th Nanotechnology Materials and Devices Conference (NMDC). IEEE. 2018, pp. 1-4.

[28] S. Sharma, D. Dixit, and K. Deka. "Deep Learning based Symbol Detection for Molecular Communications". In: 2020 IEEE International Conference on Advanced Networks and Telecommunications Systems (ANTS). 2020, pp. 1-6. DOI: 10 . 1109 / ANTS50601.2020.9342782.

[29] X. Qian, M. Di Renzo, and A. Eckford. "Molecular Communications: Model-Based and Data-Driven Receiver Design and Optimization". In: IEEE Access 7 (2019), pp. 53555-53565.
[30] Zhuangkun Wei, Weisi Guo, Bin Li, Jerome Charmet, and Chenglin Zhao. "High-dimensional metric combining for non-coherent molecular signal detection". In: IEEE Transactions on Communications 68.3 (2019), pp. 1479-1493.

[31] Nariman Farsad and Andrea Goldsmith. "Neural network detection of data sequences in communication systems". In: IEEE Transactions on Signal Processing 66.21 (2018), pp. 5663-5678.

[32] Ling-San Meng, Ping-Cheng Yeh, Kwang-Cheng Chen, and Ian F Akyildiz. "Optimal detection for diffusion-based communications in the presence of ISI". In: 2012 IEEE Global Communications Conference (GLOBECOM). IEEE. 2012, pp. 3819-3824.

[33] Hoda ShahMohammadian, Geoffrey G Messier, and Sebastian Magierowski. "Optimum receiver for molecule shift keying modulation in diffusionbased molecular communication channels". In: Nano Communication Networks 3.3 (2012), pp. 183-195.

[34] Hoda ShahMohammadian, Geoffrey G Messier, and Sebastian Magierowski. "Modelling the reception process in diffusion-based molecular communication channels". In: 2013 IEEE International Conference on Communications Workshops (ICC). IEEE. 2013, pp. 782-786.

[35] Mohammad Upal Mahfuz, Dimitrios Makrakis, and Hussein T Mouftah. "Strength based receiver architecture and communication range and rate dependent signal detection characteristics of concentration encoded molecular communication". In: 2012 Seventh International Conference on Broadband, Wireless Computing, Communication and Applications. IEEE. 2012, pp. 28-35.

[36] A Ozan Bicen, Caitlin M Austin, Ian F Akyildiz, and Craig $\mathrm{R}$ Forest. "Efficient sampling of bacterial signal transduction for detection of pulseamplitude modulated molecular signals". In: IEEE Transactions on Biomedical Circuits and Systems 9.4 (2015), pp. 505-517.

[37] Wanzhi Qiu, Thanh Cong Nguyen, and Efstratios Skafidas. "Detection of weak nano-biosensor signals corrupted by shot noise". In: 2013 13th IEEE International Conference on Nanotechnology (IEEE-NANO 2013). IEEE. 2013, pp. 305-310.

[38] Mohammad Upal Mahfuz, Dimitrios Makrakis, and Hussein T Mouftah. "A comprehensive analysis of strength-based optimum signal detection in concentration-encoded molecular communication with spike transmission". In: IEEE Transactions on NanoBioscience 14.1 (2015), pp. 67-83. 
[39] Ignacio Llatser, Albert Cabellos-Aparicio, Massimiliano Pierobon, and Eduard Alarcón. "Detection techniques for diffusion-based molecular communication". In: IEEE Journal on Selected Areas in Communications 31.12 (2013), pp. 726-734.

[40] Deniz Kilinc and Ozgur B Akan. "Receiver design for molecular communication". In: IEEE Journal on Selected Areas in Communications 31.12 (2013), pp. 705-714.

[41] Mohammad U Mahfuz, Dimitrios Makrakis, and Hussein T Mouftah. "A comprehensive study of sampling-based optimum signal detection in concentration-encoded molecular communication". In: IEEE Transactions on NanoBioscience 13.3 (2014), pp. 208-222.

[42] Ling-San Meng, Ping-Cheng Yeh, Kwang-Cheng Chen, and Ian F Akyildiz. "On receiver design for diffusion-based molecular communication". In: IEEE Transactions on Signal Processing 62.22 (2014), pp. 6032-6044.

[43] Chun Tung Chou. "A Markovian approach to the optimal demodulation of diffusion-based molecular communication networks". In: IEEE Transactions on Communications 63.10 (2015), pp. 3728-3743.

[44] Yonathan Murin, Mainak Chowdhury, Nariman Farsad, and Andrea Goldsmith. "Diversity gain of one-shot communication over molecular timing channels". In: GLOBECOM 2017-2017 IEEE Global Communications Conference. IEEE. 2017, pp. 1-6.

[45] Burcu Tepekule, Ali E Pusane, H Birkan Yilmaz, Chan-Byoung Chae, and Tuna Tugcu. "ISI mitigation techniques in molecular communication". In: IEEE Transactions on Molecular, Biological and Multi-Scale Communications 1.2 (2015), pp. 202-216.

[46] Mohammad Upal Mahfuz. "Achievable strengthbased signal detection in quantity-constrained PAM OOK concentration-encoded molecular communication". In: IEEE Transactions on NanoBioscience 15.7 (2016), pp. 619-626.

[47] Martin Damrath and Peter Adam Hoeher. "Lowcomplexity adaptive threshold detection for molecular communication". In: IEEE Transactions on NanoBioscience 15.3 (2016), pp. 200-208.

[48] Yeganeh Zamiri-Jafarian and Saeed Gazor. "Receiver design for diffusion-based molecular communication: Gaussian mixture modeling". In: 2016 IEEE International Conference on Communications (ICC). IEEE. 2016, pp. 1-6.

[49] Bin Li, Mengwei Sun, Siyi Wang, Weisi Guo, and Chenglin Zhao. "Low-complexity noncoherent signal detection for nanoscale molecular communications". In: IEEE Transactions on NanoBioscience 15.1 (2015), pp. 3-10.
[50] Adam Noel and Andrew W Eckford. "Asynchronous peak detection for demodulation in molecular communication". In: 2017 IEEE International Conference on Communications (ICC). IEEE. 2017, pp. 1-6.

[51] Satish K Tiwari, Tadi Ravi Teja Reddy, and Prabhat K Upadhyay. "Error performance optimization using logarithmic barrier function in molecular nanonetworks". In: IEEE Communications Letters 21.11 (2017), pp. 2408-2411.

[52] Bin Li, Chenglin Zhao, and Weisi Guo. "Non-linear signal detection for molecular communications". In: GLOBECOM 2017-2017 IEEE Global Communications Conference. IEEE. 2017, pp. 1-6.

[53] Ghalib H Alshammri, Mohammed S Alzaidi, Walid KM Ahmed, and Victor B Lawrence. "Lowcomplexity memory-assisted adaptive-threshold detection scheme for ON-OFF-keying diffusionbased molecular communications". In: 2017 IEEE 38th Sarnoff Symposium. IEEE. 2017, pp. 1-6.

[54] Georgia D Ntouni, Vasileios M Kapinas, and George K Karagiannidis. "On the optimal timing of detection in molecular communication systems". In: 2017 24th International Conference on Telecommunications (ICT). IEEE. 2017, pp. 1-5.

[55] Amin Zare, Ali Jamshidi, and Alireza KeshavarzHaddad. "Receiver design for pulse position modulation technique in diffusion-based molecular communication". In: 2017 IEEE 4th International Conference on Knowledge-Based Engineering and Innovation (KBEI). IEEE. 2017, pp. 0729-0733.

[56] Qingchao Li. "The clock-free asynchronous receiver design for molecular timing channels in diffusion-based molecular communications". In: IEEE Transactions on NanoBioscience 18.4 (2019), pp. 585-596.

[57] Maheshi B Dissanayake, Yansha Deng, Arumugam Nallanathan, EMN Ekanayake, and Maged Elkashlan. "Reed solomon codes for molecular communication with a full absorption receiver". In: IEEE Communications Letters 21.6 (2017), pp. 1245-1248.

[58] Vahid Jamali, Arman Ahmadzadeh, Nariman Farsad, and Robert Schober. "SCW codes for optimal CSI-free detection in diffusive molecular communications". In: 2017 IEEE International Symposium on Information Theory (ISIT). IEEE. 2017, pp. 3190-3194.

[59] Alessio Marcone, Massimiliano Pierobon, and Maurizio Magarini. "The Gaussian approximation in soft detection for molecular communication via biological circuits". In: 2017 IEEE 18th International Workshop on Signal Processing Advances in Wireless Communications (SPAWC). IEEE. 2017, pp. 1-6. 
[60] Mohammed S Alzaidi, Walid KM Ahmed, and Victor B Lawrence. "A Novel Reduced Complexity Detection Scheme for ON-OFF-Keying DiffusionBased Molecular Communications". In: 2018 International Conference on Computing, Electronics \& Communications Engineering (iCCECE). IEEE. 2018, pp. 195-200.

[61] Hao Yan, Ge Chang, Zhongke Ma, and Lin Lin. "Derivative-based signal detection for high data rate molecular communication system". In: IEEE Communications Letters 22.9 (2018), pp. 1782-1785.

[62] M. C. Gursoy and U. Mitra. "Higher Order Derivatives: Improved Pre-Processing and Receivers for Molecular Communications". In: GLOBECOM 2020 - 2020 IEEE Global Communications Conference. 2020, pp. 1-6. DOI: 10 . 1109 / GLOBECOM42002 . 2020.9322240.

[63] Vahid Jamali, Nariman Farsad, Robert Schober, and Andrea Goldsmith. "Diffusive molecular communications with reactive molecules: Channel modeling and signal design". In: IEEE Transactions on Molecular, Biological and Multi-Scale Communications 4.3 (2018), pp. 171-188.

[64] Georgia D Ntouni, Alexandros E Paschos, Vasileios M Kapinas, George K Karagiannidis, and Leontios J Hadjileontiadis. “Optimal detector design for molecular communication systems using an improved swarm intelligence algorithm". In: Micro \& Nano Letters 13.3 (2018), pp. 383-388.

[65] Vahid Jamali, Nariman Farsad, Robert Schober, and Andrea Goldsmith. "Non-coherent detection for diffusive molecular communication systems". In: IEEE Transactions on Communications 66.6 (2018), pp. 2515-2531.

[66] Murat Kuscu and Ozgur B Akan. "Maximum likelihood detection with ligand receptors for diffusion-based molecular communications in Internet of bio-nano things". In: IEEE Transactions on NanoBioscience 17.1 (2018), pp. 44-54.

[67] Giulia Muzio, Murat Kuscu, and Ozgur B Akan. "Selective signal detection with ligand receptors under interference in molecular communications". In: 2018 IEEE 19th International Workshop on Signal Processing Advances in Wireless Communications (SPAWC). IEEE. 2018, pp. 1-5.

[68] Malcolm Egan, Trung Q Duong, and Marco Di Renzo. "Biological circuits for detection in MoSKbased molecular communication". In: IEEE Access 7 (2019), pp. 21094-21102.

[69] Bin Li, Weisi Guo, Xiang Wang, Yansha Deng, Yueheng Lan, Chenglin Zhao, and Arumugam Nallanathan. "CSI-Independent Non-Linear Signal Detection in Molecular Communications". In: IEEE Transactions on Signal Processing 68 (2019), pp. 97-112.
[70] Nithin V Sabu and Abhishek K Gupta. "Detection Probability in a Molecular Communication via Diffusion System with Multiple Fully-absorbing Receivers". In: IEEE Communications Letters (2020).

[71] Mahendra S Thakur, Sanjeev Sharma, and Vimal Bhatia. "Iterative Signal Detection for Diffusion-Based Molecular Communications". In: IEEE Transactions on Molecular, Biological and Multi-Scale Communications 6.1 (2020), pp. 36-49.

[72] M. S. Thakur, S. Sharma, and V. Bhatia. "Molecular Signal Detection Using Nonlinear Mapping". In: IEEE Wireless Communications Letters (2020), pp. 1-1. DoI: 10.1109/LWC. 2020.3037184.

[73] Trang Ngoc Cao, Nikola Zlatanov, Phee Lep Yeoh, and Jamie S Evans. "Optimal detection interval for absorbing receivers in molecular communication systems with interference". In: IEEE Transactions on Molecular, Biological and Multi-Scale Communications (2020).

[74] Satish K Tiwari and Prabhat K Upadhyay. "Estimate-and-forward relaying in diffusionbased molecular communication networks: Performance evaluation and threshold optimization". In: IEEE Transactions on Molecular, Biological and Multi-Scale Communications 3.3 (2017), pp. 183-193.

[75] Sanjeev Sharma, Kuntal Deka, and Vimal Bhatia. "Thresholdless detection of symbols in nanocommunication systems". In: IEEE Transactions on NanoBioscience 19.2 (2019), pp. 259-266.

[76] X. Chen, Y. Huang, L. -L. Yang, and M. Wen. “Generalized Molecular-Shift Keying (GMoSK): Principles and Performance Analysis". In: IEEE Transactions on Molecular, Biological and Multi-Scale Communications 6.3 (2020), pp. 168-183. DoI: 10.1109/TMBMC . 2020.3021281.

[77] Jiaxing Wang, Shuo Yuan, and Mugen Peng. "Performance analysis and optimal signal detection for amplify-and-forward relay in diffusionbased molecular communication systems". In: 2017 IEEE/CIC International Conference on Communications in China (ICCC). IEEE. 2017, pp. 1-6.

[78] Nooshin Tavakkoli, Paeiz Azmi, and Nader Mokari. "Optimal positioning of relay node in cooperative molecular communication networks". In: IEEE Transactions on Communications 65.12 (2017), pp. 5293-5304.

[79] P. Hou, A. W. Eckford, and L. Zhao. "Analysis and Design of Two-Hop Diffusion-Based Molecular Communication With Ligand Receptors". In: IEEE Access 8 (2020), pp. 189458-189470. DOI: 10.1109/ACCESS . 2020.3032009. 
[80] Bon Hong Koo, H Birkan Yilmaz, Chan-Byoung Chae, and Andrew Eckford. "Detection algorithms for molecular MIMO". In: 2015 IEEE International Conference on Communications (ICC). IEEE. 2015, pp. 1122-1127.

[81] Yu Huang, Xuan Chen, Miaowen Wen, Lie-Liang Yang, Chan-Byoung Chae, and Fei Ji. "A Rising Edge-Based Detection Algorithm for MIMO Molecular Communication". In: IEEE Wireless Communications Letters 9.4 (2019), pp. 523-527.

[82] C. Wu, L. Lin, W. Guo, and H. Yan. "Signal Detection for Molecular MIMO Communications With Asymmetrical Topology". In: IEEE Transactions on Molecular, Biological and Multi-Scale Communications 6.1 (2020), pp. 60-70. DoI: 10.1109/TMBMC . 2020.3004030 .

[83] Hamid Khoshfekr Rudsari, Mohammad Reza Javan, Mahdi Orooji, Nader Mokari, and Eduard A Jorswieck. "Multiple-type transmission multipletype reception framework on molecular communication". In: IEEE Wireless Communications Letters (2020).

[84] N. V. Sabu, N. Varshney, and A. K. Gupta. "3-D Diffusive Molecular Communication With Two FullyAbsorbing Receivers: Hitting Probability and Performance Analysis". In: IEEE Transactions on Molecular, Biological and Multi-Scale Communications 6.3 (2020), pp. 244-249. DOI: 10 . 1109 / TMBMC . 2020.3031414.

[85] Yuting Fang, Adam Noel, Nan Yang, Andrew W Eckford, and Rodney A Kennedy. "Distributed cooperative detection for multi-receiver molecular communication". In: 2016 IEEE Global Communications Conference (GLOBECOM). IEEE. 2016, pp. 1-7.

[86] Siavash Ghavami and Farshad Lahouti. "Abnormality detection in correlated Gaussian molecular nano-networks: Design and analysis". In: IEEE transactions on nanobioscience 16.3 (2017), pp. 189-202.

[87] Yuting Fang, Adam Noel, Nan Yang, Andrew W Eckford, and Rodney A Kennedy. "Convex optimization of distributed cooperative detection in multi-receiver molecular communication". In: IEEE Transactions on Molecular, Biological and Multi-Scale Communications 3.3 (2017), pp. 166-182.

[88] Reza Mosayebi, Vahid Jamali, Nafiseh Ghoroghchian, Robert Schober, Masoumeh Nasiri-Kenari, and Mahdieh Mehrabi. "Cooperative abnormality detection via diffusive molecular communications". In: IEEE Transactions on NanoBioscience 16.8 (2017), pp. 828-842.
[89] Sinem Solak and Mengüç Öner. "Sequential Decision Fusion for Abnormality Detection via Diffusive Molecular Communications". In: IEEE Communications Letters (2020).

[90] Yuting Fang, Adam Noel, Yiran Wang, and Nan Yang. "Simplified cooperative detection for multireceiver molecular communication". In: 2017 IEEE Information Theory Workshop (ITW). IEEE. 2017, pp. 329-333.

[91] Reza Mosayebi, Wayan Wicke, Vahid Jamali, Arman Ahmadzadeh, Robert Schober, and Masoumeh Nasiri-Kenari. "Advanced target detection via molecular communication". In: 2018 IEEE Global Communications Conference (GLOBECOM). IEEE. 2018, pp. 1-7.

[92] Pengfeng Hou, Liqiang Zhao, and Andrew W Eckford. "Improving receiver performance of diffusion-based molecular MIMO communication". In: 2018 IEEE 18th International Conference on Nanotechnology (IEEE-NANO). IEEE. 2018, pp. 1-4.

[93] Yuting Fang, Adam Noel, Nan Yang, Andrew W Eckford, and Rodney A Kennedy. "Maximum likelihood detection for cooperative molecular communication". In: 2018 IEEE International Conference on Communications (ICC). IEEE. 2018, pp. 1-7.

[94] Nafiseh Ghoroghchian, Mahtab Mirmohseni, and Masoumeh Nasiri-Kenari. "Abnormality Detection and Monitoring in Multi-Sensor Molecular Communication". In: IEEE Transactions on Molecular, Biological and Multi-Scale Communications 5.2 (2019), pp. 68-83.

[95] Yuting Fang, Adam Noel, Nan Yang, Andrew W Eckford, and Rodney A Kennedy. "Symbol-bysymbol maximum likelihood detection for cooperative molecular communication". In: IEEE Transactions on Communications 67.7 (2019), pp. 4885-4899.

[96] Ghalib H Alshammri, Walid KM Ahmed, and Victor B Lawrence. "Receiver Techniques for DiffusionBased Molecular Nano Communications Using an Adaptive Neuro-Fuzzy-Based Multivariate Polynomial Approximation". In: IEEE Transactions on Molecular, Biological and Multi-Scale Communications 4.3 (2018), pp. 140-159.

[97] S. N. Solak and M. Oner. "Neural Network Based Decision Fusion for Abnormality Detection via Molecular Communications". In: 2020 IEEE Workshop on Signal Processing Systems (SiPS). 2020, pp. 1-5. DOI: 10 . 1109 / SiPS50750 . 2020 . 9195212. 
[98] Wei-An Lin, Yen-Chi Lee, Ping-Cheng Yeh, and Chia-han Lee. "Signal detection and ISI cancellation for quantity-based amplitude modulation in diffusion-based molecular communications". In: 2012 IEEE Global Communications Conference (GLOBECOM). IEEE. 2012, pp. 4362-4367.

[99] Adam Noel, Karen C Cheung, and Robert Schober. "Diffusive molecular communication with disruptive flows". In: 2014 IEEE International Conference on Communications (ICC). IEEE. 2014, pp. 3600-3606.

[100] Jeongman Lee, Minhoe Kim, and Dong-Ho Cho. "Asynchronous detection algorithm for diffusionbased molecular communication in timing modulation channel". In: IEEE Communications Letters 19.12 (2015), pp. 2114-2117.

[101] Yang-Kai Lin, Wei-An Lin, Chia-Han Lee, and PingCheng Yeh. "Asynchronous threshold-based detection for quantity-type-modulated molecular communication systems". In: IEEE Transactions on Molecular, Biological and Multi-Scale Communications 1.1 (2015), pp. 37-49.

[102] Uri Rogers and Min-Sung Koh. "Parallel molecular distributed detection with Brownian motion". In: IEEE Transactions on NanoBioscience 15.8 (2016), pp. 871-880.

[103] Junzhe Xiong and Hui Li. "Receiver design for binary timing-based molecular communication". In: 2016 8th International Conference on Wireless Communications \& Signal Processing (WCSP). IEEE. 2016, pp. 1-6.

[104] Toshiaki Koike-Akino, Junichi Suzuki, and Philip V Orlik. "Molecular signaling design exploiting cyclostationary drift-diffusion fluid". In: GLOBECOM 2017-2017 IEEE Global Communications Conference. IEEE. 2017, pp. 1-7.

[105] Vahid Jamali, Arman Ahmadzadeh, and Robert Schober. "On the design of matched filters for molecule counting receivers". In: IEEE Communications Letters 21.8 (2017), pp. 1711-1714.

[106] Nariman Farsad and Andrea Goldsmith. "Sliding bidirectional recurrent neural networks for sequence detection in communication systems". In: 2018 IEEE International Conference on Acoustics, Speech and Signal Processing (ICASSP). IEEE. 2018, pp. 2331-2335.

[107] Mustafa Can Gursoy, Ertugrul Basar, Ali Emre Pusane, and Tuna Tugcu. "Index modulation for molecular communication via diffusion systems". In: IEEE Transactions on Communications 67.5 (2019), pp. 3337-3350.
[108] Qingchao Li. "The asymmetric-distance metrics for decoding of convolutional codes in diffusionbased molecular communications". In: IEEE Transactions on NanoBioscience 18.3 (2019), pp. 469-481.

[109] Jiaxing Wang, Mugen Peng, Yaqiong Liu, Xiqing Liu, and Mahmoud Daneshmand. "Performance Analysis of Signal Detection for Amplify-andForward Relay in Diffusion-Based Molecular Communication Systems". In: IEEE Internet of Things Journal 7.2 (2019), pp. 1401-1412.

[110] Mahendra S Thakur, Sandesh Jain, Sanjeev Sharma, and Vimal Bhatia. "Nonlinear Receiver for Diffusion-Based Molecular Communications". In: 2019 IEEE 30th Annual International Symposium on Personal, Indoor and Mobile Radio Communications (PIMRC). IEEE. 2019, pp. 1-6.

[111] Shenghan Liu, Zhuangkun Wei, Bin Li, and Chenglin Zhao. "Unsupervised Clustering-based Non-Coherent Detection for Molecular Communications". In: IEEE Communications Letters (2020).

[112] Amit Singhal, Ranjan K Mallik, and Brejesh Lall. "Molecular communication with brownian motion and a positive drift: performance analysis of amplitude modulation schemes". In: IET Communications 8.14 (2014), pp. 2413-2422.

[113] Adam Noel, Karen C Cheung, and Robert Schober. "Optimal receiver design for diffusive molecular communication with flow and additive noise". In: IEEE Transactions on NanoBioscience 13.3 (2014), pp. 350-362.

[114] Ardalan Alizadeh, Hamid Reza Bahrami, Mehdi Maleki, Nghi H Tran, and Pedram Mohseni. "On the coexistence of nano networks: Sensing techniques for molecular communications". In: IEEE Transactions on Molecular, Biological and MultiScale Communications 3.4 (2017), pp. 209-223.

[115] Georgia D Ntouni, Vasileios M Kapinas, and George K Karagiannidis. "Optimization of the detection process timing in molecular communication systems with flow". In: 201725 th Telecommunication Forum (TELFOR). IEEE. 2017, pp. 1-4.

[116] Manav R Bhatnagar et al. "3-D diffusive-drift molecular channel characterization for active and passive receivers". In: IEEE Transactions on Molecular, Biological and Multi-Scale Communications 4.2 (2018), pp. 107-117.

[117] Arman Ahmadzadeh, Vahid Jamali, Adam Noel, and Robert Schober. "Diffusive mobile molecular communications over time-variant channels". In: IEEE Communications Letters 21.6 (2017), pp. $1265-1268$. 
[118] Ge Chang, Lin Lin, and Hao Yan. "Adaptive detection and ISI mitigation for mobile molecular communication". In: IEEE Transactions on NanoBioscience 17.1 (2017), pp. 21-35.

[119] Xiaodong Mu, Hao Yan, Bin Li, Manhua Liu, Ruifeng Zheng, Yan Li, and Lin Lin. "LowComplexity Adaptive Signal Detection for Mobile Molecular Communication". In: IEEE Transactions on NanoBioscience 19.2 (2020), pp. 237-248.

[120] Lokendra Chouhany, Neeraj Varshney, and Prabhat Kumar Sharma. “On Gradient Descent Optimization in Diffusion-Advection based 3-D Molecular Cooperative Communication". In: IEEE Transactions on NanoBioscience (2020).

[121] Jiaxing Wang, Xiqing Liu, Mugen Peng, and Mahmoud Daneshmand. "Performance Analysis of DMoSK Modulation in Mobile Diffusive-Drift Molecular Communications". In: IEEE Internet of Things Journal (2020).

[122] Yonathan Murin, Nariman Farsad, Mainak Chowdhury, and Andrea Goldsmith. "Optimal detection for one-shot transmission over diffusion-based molecular timing channels". In: IEEE Transactions on Molecular, Biological and Multi-Scale Communications 4.2 (2018), pp. 43-60.

[123] Manav R Bhatnagar et al. "M-ary Poisson reception in molecular communication". In: Micro \& Nano Letters 13.4 (2018), pp. 509-513.

[124] Wayan Wicke, Arman Ahmadzadeh, Vahid Jamali, Harald Unterweger, Christoph Alexiou, and Robert Schober. "Magnetic nanoparticle-based molecular communication in microfluidic environments". In: IEEE Transactions on NanoBioscience 18.2 (2019), pp. 156-169.

[125] Qingchao Li. "A Novel Time-Based Modulation Scheme in Time-Asynchronous Channels for Molecular Communications". In: IEEE Transactions on NanoBioscience 19.1 (2019), pp. 59-67.

[126] Yanbin Chen, Yu Li, Lin Lin, and Hao Yan. "Parameter Estimation of Diffusive Molecular Communication With Drift". In: IEEE Access 8 (2020), pp. 142704-142713.

[127] Fardad Vakilipoor, Francesca Ratti, Maurizio Magarini, and Hamdan Awan. "Linear Receiver Design for Time-Varying Poisson Molecular Communication Channels with Memory". In: 2020 IEEE 21st International Workshop on Signal Processing Advances in Wireless Communications (SPAWC). IEEE. 2020, pp. 1-5.

[128] Nooshin Tavakkoli, Paeiz Azmi, and Nader Mokari. "Performance evaluation and optimal detection of relay-assisted diffusion-based molecular communication with drift". In: IEEE Transactions on NanoBioscience 16.1 (2016), pp. 34-42.
[129] Satish K Tiwari and Prabhat K Upadhyay. "Estimate-and-Forward Relaying in Molecular Communication using Brownian Motion with Drift". In: 2018 International Conference on Signal Processing and Communications (SPCOM). IEEE. 2018, pp. 502-506.

[130] Hamid Khoshfekr Rudsari, Nader Mokari, Mohammad Reza Javan, Eduard A Jorswieck, and Mahdi Orooji. "Drug Release Management for Dynamic TDMA-Based Molecular Communication". In: IEEE Transactions on Molecular, Biological and Multi-Scale Communications 5.3 (2019), pp. 233-246.

[131] Uri Rogers and Min-Sung Koh. "Exploring molecular distributed detection". In: 2015 9th IEEE International Conference on Nano/Molecular Medicine \& Engineering (NANOMED). IEEE. 2015, pp. 153-158.

[132] Trang C Mai, Malcolm Egan, Trung Q Duong, and Marco Di Renzo. "Event detection in molecular communication networks with anomalous diffusion". In: IEEE Communications Letters 21.6 (2017), pp. 1249-1252.

[133] Shuai Huang, Lin Lin, Hao Yan, Juan $\mathrm{Xu}$, and Fuqiang Liu. "Statistical analysis of received signal and error performance for mobile molecular communication". In: IEEE Transactions on NanoBioscience 18.3 (2019), pp. 415-427.

[134] S. Md Mustam, S. K. Syed-Yusof, and S. Zubair. "Capacity and Delay Spread in Multilayer DiffusionBased Molecular Communication (DBMC) Channel". In: IEEE Transactions on NanoBioscience 15.7 (2016), pp. 599-612. DoI: 10 . 1109 / TNB . 2016 . 2631240

[135] Bin Li, Mengwei Sun, Siyi Wang, Weisi Guo, and Chenglin Zhao. "Local convexity inspired low-complexity noncoherent signal detector for nanoscale molecular communications". In: IEEE Transactions on Communications 64.5 (2016), pp. 2079-2091.

[136] Amit Kumar Shrivastava, Debanjan Das, and Rajarshi Mahapatra. "Adaptive Threshold Detection and ISI Mitigation in Mobile Molecular Communication". In: 2020 IEEE Wireless Communications and Networking Conference (WCNC). IEEE. 2020, pp. 1-6.

[137] Shuai Huang, Lin Lin, Weisi Guo, Hao Yan, Juan $\mathrm{Xu}$, and Fuqiang Liu. "Initial Distance Estimation and Signal Detection for Diffusive Mobile Molecular Communication". In: IEEE Transactions on NanoBioscience (2020).

[138] Arzhang Shahbazi and Ali Jamshidi. "Improving adaptive receivers performance in molecular communication via diffusion". In: IET Nanobiotechnology 13.4 (2019), pp. 441-448. 
[139] Neeraj Varshney, Aditya K Jagannatham, and Pramod K Varshney. "On diffusive molecular communication with mobile nanomachines". In: 2018 52nd Annual Conference on Information Sciences and Systems (CISS). IEEE. 2018, pp. 1-6.

[140] Amit Kumar Shrivastava, Debanjan Das, and Rajarshi Mahapatra. "Slope Based Detection for Mobile Molecular Communication Based on Einstein's Law of Diffusion". In: 2018 15th IEEE India Council International Conference (INDICON). IEEE. 2018, pp. 1-6.

[141] Peng He, Bowen Pi, and Qiang Liu. "Calcium signaling in mobile molecular communication networks: From a multimedia view". In: IEEE Access 7 (2019), pp. 164825-164834.

[142] Yutaka Okaie. "Cluster Formation by Mobile Molecular Communication Systems". In: IEEE Transactions on Molecular, Biological and MultiScale Communications 5.2 (2019), pp. 153-157.

[143] Trang Ngoc Cao, Arman Ahmadzadeh, Vahid Jamali, Wayan Wicke, Phee Lep Yeoh, Jamie Evans, and Robert Schober. "Diffusive Mobile MC with Absorbing Receivers: Stochastic Analysis and Applications". In: IEEE Transactions on Molecular, Biological and Multi-Scale Communications 5.2 (2019), pp. 84-99.

[144] Mahendra S Thakur, Sanjeev Sharma, and Vimal Bhatia. "Noncoherent Detection for Dynamic Transmitter and Receiver in Molecular Communication". In: 2018 IEEE International Conference on Advanced Networks and Telecommunications Systems (ANTS). IEEE. 2018, pp. 1-6.

[145] Chunfu Luo, Xia Wu, Lin Lin, Chao Wang, and Fuqiang Liu. "Non-Coherent Signal Detection Technique for Mobile Molecular Communication at High Data Rates". In: 2019 IEEE Global Communications Conference (GLOBECOM). IEEE. 2019, pp. 1-6.

[146] Amit K Shrivastava, Debanjan Das, Rajarshi Mahapatra, and Saraju P Mohanty. "dMole: A Novel Transreceiver for Mobile Molecular Communication Using Robust Differential Detection Techniques". In: IEEE Transactions on NanoBioscience 19.4 (2020), pp. 609-621.

[147] Murat Kuscu and Ozgur B Akan. "FRET-based mobile molecular nanonetworks". In: 2013 12th Annual Mediterranean Ad Hoc Networking Workshop (MED-HOC-NET). IEEE. 2013, pp. 120-127.

[148] SM Reza Rouzegar and Umberto Spagnolini. "Diffusive MIMO molecular communications: Channel estimation, equalization, and detection". In: IEEE Transactions on Communications 67.7 (2019), pp. 4872-4884.
[149] Amit K. Shrivastava, Debanjan Das, and Rajarshi Mahapatra. "Performance Evaluation of Mobile Molecular Communication System using Neural Network Detector". In: IEEE Wireless Communications Letters (2021), pp. 1-1. DoI: 10.1109/LWC. 2021.3079522.

[150] L. Sun, Y. Wang, A. L. Swindlehurst, and X. Tang. "Generative-Adversarial-Network Enabled Signal Detection for Communication Systems With Unknown Channel Models". In: IEEE Journal on Selected Areas in Communications 39.1 (2021), pp. 47-60. DOI: 10.1109/JSAC. 2020.3036954.

[151] Neeraj Varshney, Adarsh Patel, Yansha Deng, Werner Haselmayr, Pramod K Varshney, and Arumugam Nallanathan. "Abnormality detection inside blood vessels with mobile nanomachines". In: IEEE Transactions on Molecular, Biological and Multi-Scale Communications 4.3 (2018), pp. 189-194.

[152] Lokendra Chouhan, Prabhat Kumar Sharma, and Neeraj Varshney. "Optimal transmitted molecules and decision threshold for drift-induced diffusive molecular channel with mobile nanomachines". In: IEEE Transactions on NanoBioscience 18.4 (2019), pp. 651-660.

[153] Y. Tang, M. Wen, X. Chen, Y. Huang, and L. -L. Yang. "Molecular Type Permutation Shift Keying for Molecular Communication". In: IEEE Transactions on Molecular, Biological and Multi-Scale Communications 6.2 (2020), pp. 160-164. DoI: 10.1109/TMBMC . 2020.3014803.

[154] Takuya Obuchi, Yutaka Okaie, Tadashi Nakano, Takahiro Hara, and Shojiro Nishio. "Inbody mobile bionanosensor networks through non-diffusionbased molecular communication". In: 2015 IEEE International Conference on Communications (ICC). IEEE. 2015, pp. 1078-1084.

[155] Tadashi Nakano, Yutaka Okaie, Shouhei Kobayashi, Takako Koujin, Chen-Hao Chan, Yu-Hsiang Hsu, Takuya Obuchi, Takahiro Hara, Yasushi Hiraoka, and Tokuko Haraguchi. "Performance evaluation of leader-follower-based mobile molecular communication networks for target detection applications". In: IEEE Transactions on Communications 65.2 (2016), pp. 663-676.

[156] Neeraj Varshney, Adarsh Patel, Werner Haselmayr, Aditya K Jagannatham, Pramod K Varshney, and Weisi Guo. "Impact of cooperation in flowinduced diffusive mobile molecular communication". In: 2018 52nd Asilomar Conference on Signals, Systems, and Computers. IEEE. 2018, pp. 494-498. 
[157] Neeraj Varshney, Adarsh Patel, Werner Haselmayr, Aditya K Jagannatham, Pramod K Varshney, and Arumugam Nallanathan. "Impact of intermediate nanomachines in multiple cooperative nanomachine-assisted diffusion advection mobile molecular communication". In: IEEE Transactions on Communications 67.7 (2019), pp. 4856-4871.

[158] L. Khaloopour, M. Mirmohseni, and M. NasiriKenari. "Cooperative Abnormality Detection in Fluidic Medium Molecular Communication". In: 2020 Iran Workshop on Communication and Information Theory (IWCIT). 2020, pp. 1-6. DoI: 10 . 1109/IWCIT50667.2020.9163534.

[159] X. Wang, M. D. Higgins, and M. S. Leeson. “Distance Estimation Schemes for Diffusion Based Molecular Communication Systems". In: IEEE Communications Letters 19.3 (2015), pp. 399-402. DoI: 10 . 1109/LCOMM . 2014. 2387826.

[160] Z. Luo, L. Lin, Q. Fu, and H. Yan. "An Effective Distance Measurement Method for Molecular Communication Systems". In: 2018 IEEE International Conference on Sensing, Communication and Networking (SECON Workshops). 2018, pp. 1-4. DOI: 10.1109/SECONW. 2018.8396344.

[161] Z. Luo, L. Lin, W. Guo, S. Wang, F. Liu, and H. Yan. "One Symbol Blind Synchronization in SIMO Molecular Communication Systems". In: IEEE Wireless Communications Letters 7.4 (2018), pp. 530-533. DoI: 10.1109/LWC. 2018.2793197.

[162] H. ShahMohammadian, G. G. Messier, and S. Magierowski. "Blind Synchronization in Diffusion-Based Molecular Communication Channels". In: IEEE Communications Letters 17.11 (2013), pp. 2156-2159. DOI: 10 . 1109 / LCOMM . 2013.100713 .131727$.

[163] V. Jamali, A. Ahmadzadeh, and R. Schober. "Symbol Synchronization for Diffusion-Based Molecular Communications". In: IEEE Transactions on NanoBioscience 16.8 (2017), pp. 873-887. DoI: 10.1109/TNB . 2017.2782761.

[164] L. Lin, W. Li, R. Zheng, F. Liu, and H. Yan. "Diffusion-Based Reference Broadcast Synchronization for Molecular Communication in Nanonetworks". In: IEEE Access 7 (2019), pp. 95527-95535. DOI: 10 . 1109/ ACCESS . 2019. 2929873.

[165] L. Huang, L. Lin, F. Liu, and H. Yan. "Clock Synchronization for Mobile Molecular Communication Systems". In: IEEE Transactions on NanoBioscience (2020), pp. 1-1. DOI: 10 .1109/TNB . 2020. 3041631.
[166] Lin Qiu, Qiangyuan Zhu, Yibo Gao, Bingwen Yu, Hao Ren, Wei Jin, Qinhan Jin, and Ying Mu. "Selfpriming compartmentalization digital LAMP for single molecule detection". In: 2012 Asia Communications and Photonics Conference (ACP). IEEE. 2012, pp. 1-3.

[167] Quan Wang and Behrouz Arash. "Nanoresonators in sensors and molecular transportation: An introduction to the possibilities of carbon nanotubes and graphene sheets". In: IEEE Nanotechnology Magazine 8.4 (2014), pp. 29-37.

[168] Mohiuddin Munna, Md Obidul Islam, Md Kabiruzzaman, and Zahid Hasan Mahmood. "Performance analysis of a Si-NW biosensor for detection of charged biomolecules". In: 2014 International Conference on Informatics, Electronics \& Vision (ICIEV). IEEE. 2014, pp. 1-5.

[169] Milan Sasmal, Tapas Kumar Maiti, and Tarun Kanti Bhattacharyya. "Conjugation of Bovine Serum Albumin With ZnO Nanosphere-A Novel Approach for Ultra-Low Level Mercury Ion Detection". In: IEEE Transactions on NanoBioscience 15.7 (2016), pp. 748-755.

[170] Laura Grebenstein, Jens Kirchner, Renata Stavracakis Peixoto, Wiebke Zimmermann, Florian Irnstorfer, Wayan Wicke, Arman Ahmadzadeh, Vahid Jamali, Georg Fischer, Robert Weigel, et al. "Biological optical-to-chemical signal conversion interface: A small-scale modulator for molecular communications". In: IEEE Transactions on NanoBioscience 18.1 (2018), pp. 31-42.

[171] Laura Grebenstein, Jens Kirchner, Wayan Wicke, Arman Ahmadzadeh, Vahid Jamali, Georg Fischer, Robert Weigel, Andreas Burkovski, and Robert Schober. "A Molecular Communication Testbed Based on Proton Pumping Bacteria: Methods and Data". In: IEEE Transactions on Molecular, Biological and Multi-Scale Communications 5.1 (2019), pp. 56-62.

[172] Z Cao, C Qin, L Bi, Y Chen, Z Xie, and B Yao. "Graphene enhanced intra-resonator biochemical detection with individual molecule sensitivity and selectivity". In: 2019 18th International Conference on Optical Communications and Networks (ICOCN). IEEE. 2019, pp. 1-3.

[173] Chengjie Zhu, Yuhan Wen, Tao Liu, Haw Yang, and Kaushik Sengupta. "A Packaged Ingestible Bio-Pill with 15-Pixel Multiplexed Fluorescence NucleicAcid Sensor and Bi-Directional Wireless Interface for In-Vivo Bio-Molecular Sensing". In: 2020 IEEE Symposium on VLSI Circuits. IEEE. 2020, pp. 1-2.

[174] Na-Rae Kim, Nariman Farsad, Chan-Byoung Chae, and Andrew W Eckford. "A universal channel model for molecular communication systems with metal-oxide detectors". In: 2015 IEEE International Conference on Communications (ICC). IEEE. 2015, pp. 1054-1059. 
[175] Changmin Lee, Bonhong Koo, Na-Rae Kim, Birkan Yilmaz, Nariman Farsad, Andrew Eckford, and Chan-Byoung Chae. "Molecular MIMO communication link". In: 2015 IEEE Conference on Computer Communications Workshops (INFOCOM WKSHPS). IEEE. 2015, pp. 13-14.

[176] Bon-Hong Koo, Changmin Lee, H Birkan Yilmaz, Nariman Farsad, Andrew Eckford, and ChanByoung Chae. "Molecular MIMO: From theory to prototype". In: IEEE Journal on Selected Areas in Communications 34.3 (2016), pp. 600-614.

[177] Nariman Farsad, David Pan, and Andrea Goldsmith. "A novel experimental platform for in-vessel multi-chemical molecular communications". In: GLOBECOM 2017-2017 IEEE Global Communications Conference. IEEE. 2017, pp. 1-6.

[178] Haoyang Zhai, Qiang Liu, Athanasios V Vasilakos, and Kun Yang. "Anti-ISI demodulation scheme and its experiment-based evaluation for diffusionbased molecular communication". In: IEEE Transactions on NanoBioscience 17.2 (2018), pp. 126-133.

[179] Haoyang Zhai, Liting Yang, Tadashi Nakano, Qiang Liu, and Kun Yang. "Bio-inspired design and implementation of mobile molecular communication systems at the macroscale". In: 2018 IEEE Global Communications Conference (GLOBECOM). IEEE. 2018, pp. 1-6.

[180] Padarthi Naga Prasanth, Kakani Pruthvi Sumanth, Vijay K Chakka, and Gouriprasanna Roy. "Experimental Implementation of Molecular Communication System using Sampling based Adaptive Threshold Variation Demodulation Algorithm". In: 2018 IEEE International Conference on Advanced Networks and Telecommunications Systems (ANTS). IEEE. 2018, pp. 1-5.

[181] Ladan Khaloopour, Seyed Vahid Rouzegar, Alireza Azizi, Amir Hosseinian, Maryam FarahnakGhazani, Nahal Bagheri, Mahtab Mirmohseni, Hamidreza Arjmandi, Reza Mosayebi, and Masoumeh Nasiri-Kenari. "An Experimental Platform for Macro-Scale Fluidic Medium Molecular Communication". In: IEEE Transactions on Molecular, Biological and Multi-Scale Communications 5.3 (2019), pp. 163-175.

[182] Na-Rae Kim, Nariman Farsad, Changmin Lee, Andrew W Eckford, and Chan-Byoung Chae. "An experimentally validated channel model for molecular communication systems". In: IEEE Access 7 (2019), pp. 81849-81858.
[183] Doaa Ahmed, Harald Unterweger, Georg Fischer, Robert Schobe, and Jens Kirchner. "Characterization of an Inductance-based Detector in Molecular Communication Testbed Based on Superparamagnetic Iron Oxide Nanoparticles". In: 2019 IEEE SENSORS. IEEE. 2019, pp. 1-4.

[184] Chun Tung Chou. "Designing molecular circuits for approximate maximum a posteriori demodulation of concentration modulated signals". In: IEEE Transactions on Communications 67.8 (2019), pp. 5458-5473.

[185] Daniel Tunç Mcguiness, Stamatios Giannoukos, Stephen Taylor, and Alan Marshall. "Experimental and Analytical Analysis of Macro-Scale Molecular Communications Within Closed Boundaries". In: IEEE Transactions on Molecular, Biological and Multi-Scale Communications 5.1 (2019), pp. 44-55.

[186] Bon-Hong Koo, Ho Joong Kim, Jang-Yeon Kwon, and Chan-Byoung Chae. "Deep Learning-based Human Implantable Nano Molecular Communications". In: ICC 2020-2020 IEEE International Conference on Communications (ICC). IEEE. 2020, pp. 1-7.

[187] A Oguz Kislal, Bayram Cevdet Akdeniz, Changmin Lee, Ali E Pusane, Tuna Tugcu, and Chan-Byoung Chae. "ISI-Mitigating Channel Codes for Molecular Communication Via Diffusion". In: IEEE Access 8 (2020), pp. 24588-24599.

[188] Mahmoud Abbaszadeh, Iresha Atthanayake, Peter J Thomas, and Weisi Guo. "Molecular Signal Tracking and Detection Methods in Fluid Dynamic Channels". In: IEEE Transactions on Molecular, Biological and Multi-Scale Communications (2020).

[189] Li Sun and Yuwei Wang. "CTBRNN: A novel deeplearning based signal sequence detector for communications systems". In: IEEE Signal Processing Letters 27 (2019), pp. 21-25.

[190] Shicai Xu, Jian Zhan, Baoyuan Man, Shouzhen Jiang, Weiwei Yue, Shoubao Gao, Chengang Guo, Hanping Liu, Zhenhua Li, Jihua Wang, et al. "Realtime reliable determination of binding kinetics of DNA hybridization using a multi-channel graphene biosensor". In: Nature communications 8.1 (2017), pp. 1-10.

[191] Yasuhide Ohno, Kenzo Maehashi, and Kazuhiko Matsumoto. "Label-free biosensors based on aptamer-modified graphene field-effect transistors". In: Journal of the American Chemical Society 132.51 (2010), pp. 18012-18013.

[192] Shimaa Eissa and Mohammed Zourob. "In vitro selection of DNA aptamers targeting $\beta$-lactoglobulin and their integration in graphene-based biosensor for the detection of milk allergen". In: Biosensors and Bioelectronics 91 (2017), pp. 169-174. 
[193] Byeongju Kim, Hyun Seok Song, Hye Jun Jin, Eun Jin Park, Sang Hun Lee, Byung Yang Lee, Tai Hyun Park, and Seunghun Hong. "Highly selective and sensitive detection of neurotransmitters using receptor-modified single-walled carbon nanotube sensors". In: Nanotechnology 24.28 (2013), p. 285501.

[194] Fernando Patolsky, Gengfeng Zheng, and Charles M Lieber. Nanowire-based biosensors. 2006.

[195] Yinxi Huang, Xiaochen Dong, Yumeng Shi, Chang Ming Li, Lain-Jong Li, and Peng Chen. "Nanoelectronic biosensors based on CVD grown graphene". In: Nanoscale 2.8 (2010), pp. 1485-1488.

[196] Nithin V Sabu, Neeraj Varshney, and Abhishek K Gupta. "3-D Diffusive Molecular Communication with Two Fully-Absorbing Receivers: Hitting Probability and Performance Analysis". In: IEEE Transactions on Molecular, Biological and MultiScale Communications 6.3 (2020), pp. 244-249.

[197] Nithin V Sabu, Neeraj Varshney, and Abhishek K Gupta. "On the Performance of the Primary and Secondary Links in a 3-D Underlay Cognitive Molecular Communication". In: arXiv preprint arXiv:2102.05812 (2021).

[198] S. Sun, Z. Cao, H. Zhu, and J. Zhao. "A Survey of Optimization Methods From a Machine Learning Perspective". In: IEEE Transactions on Cybernetics 50.8 (2020), pp. 3668-3681. DOI: 10.1109/TCYB . 2019.2950779.

\section{AUTHORS}

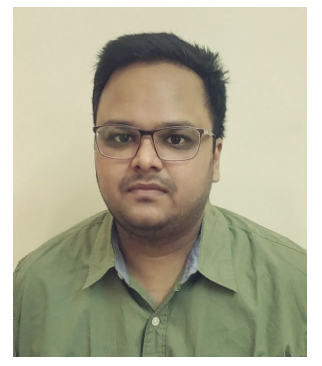

Amit K. Shrivastava received a B.Tech. degree in Electronics and Communication engineering from the National Institute of Technology, Raipur in 2010; an M.Tech. degree in Digital Communication from Atal Bihari Vajpayee-Indian Institute of Information Technology and Management, Gwalior, in 2013, and is currently pursuing Ph.D. degree in Electronics and Communication engineering from Dr. SPM IIIT Naya Raipur.

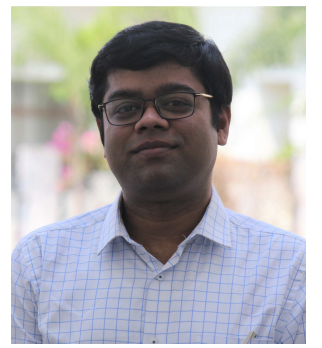

Debanjan Das received a B.Tech. degree in applied Electronics and Instrumentation engineering from the Heritage Institute of Technology, Kolkata, in 2009, an M.Tech. degree in Instrumentation from Indian Institute of Technology, Kharagpur, in 2011, and a Ph.D. degree in Electrical Engineering from Indian Institute of Technology, Kharagpur, in 2016. $\mathrm{He}$ is an Assistant Professor with Dr. SPM IIIT Naya Raipur.

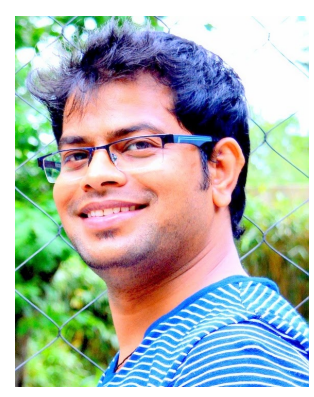

Neeraj Varshney received a B.Tech. degree in Electronics and Communication engineering from Uttar Pradesh Technical University, Lucknow, India, in 2008, an M.Tech. degree in electronics and communication engineering from the Jaypee Institute of Information Technology, Noida, India, in 2011, and a Ph.D. degree in electrical engineering from Indian Institute of Technology Kanpur, India, in 2018. Since Nov' 18 he is a international research associate with the wireless networks division at National Institute of Standards and Technology, Maryland, USA. His research interests are in signal processing, communications and networks which include mmWave and $\mathrm{THz}$ wireless communication, MIMO technology, molecular communication. From Oct '11 to Aug '12, he was a project research fellow at Jaypee Institute of Information Technology Noida, India. From May '18 to Sep '18, he was a visiting researcher with the department of electrical engineering and computer science at Syracuse University, New York, USA. He has served as a TPC member for 2019/2020 IEEE Globecom conferences in the track on Molecular, Biological, and Multi-scale Communications.

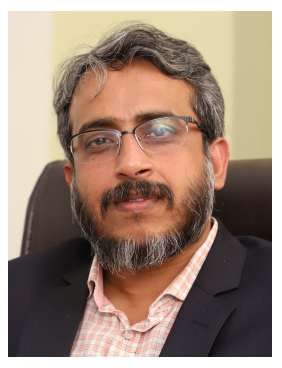

Rajarshi Mahapatra received a Ph.D. degree in Electronics and Electrical Communication engineering from the Indian Institute of Technology Kharagpur, Kharagpur, India, and a postdoctoral degree from the Commissariat à l'Énergie Atomique et aux Énergies, Grenoble, France. He is Associate Professor with the Department of Electronics and Communication Engineering, Dr. SPM IIIT Naya Raipur. He is also handling the responsibilities of Dean (Academics) of IIITNR. 